\title{
Algebras of General Convexity and General Concavity, Nonlinear Programming, Physics, and Inequalities
}

\author{
Milan R. TASKović
}

\begin{abstract}
In this paper we introduced the fundamental elements of new algebras of general convexity and general concavity. This paper presents essential elements of a new general convex (concave) functional analysis. Applications in linear and nonlinear functional analysis, linear and nonlinear programming, physics, and inequalities are considered. Adequate to fundamental fact that every set has three own sides (upper, lower and middle) we introduced and consider three relativistic physics (general convex, general concave and middle) and three physics in the classical sense. In this context we obtain relativistic Einstein's theory as a relativistic physics on the middle transversal linear spaces.
\end{abstract}

\section{Algebras of General Convexity}

Algebra of General Convexity. Let $X$ and $Y$ be arbitrary nonempty sets and let $A: Y \times Y \rightarrow \mathbb{R}_{+}^{0}:=[0,+\infty)$ be a function. In this part we considered two nonempty sets $X$ and $Y$ with a general convex structure denoted by $G(x, y, \lambda)$ iff there exist a mapping $G: X \times X \times I \rightarrow Y$ (for the closed unit interval $I:=[0,1])$ and a bisection function $g:\left(\mathbb{R}_{+}^{0}\right)^{2} \rightarrow \mathbb{R}_{+}^{0}$ satisfying the following inequality

$$
A(z, G(x, y, \lambda)) \leq \max \{A(z, x), A(z, y), g(A(z, x), A(z, y))\}
$$

for all $x, y \in X$ and $z \in Y$ with an arbitrary $\lambda \in[0,1]$. Similarly, a nonempty set $X$ is with a general affine structure denoted by $G(x, y, \lambda)$ iff there exists a mapping $G: X \times X \times \mathbb{R} \rightarrow Y$ such that (S) for all $x, y \in X$ and $z \in Y$ with an arbitrary $\lambda \in \mathbb{R}$. In the case if $X=Y$, a subset $K$ of $Y$ is general convex iff $G(x, y, \lambda) \in K$ for all $x, y \in K$ and an arbitrary $\lambda \in[0,1]$. See Figure 1 .

2000 Mathematics Subject Classification. Primary: 04A25, 54E15, 47H10, 05A15. Secondary: 54H25, 54E24.

Key words and phrases. General convex structures, general convex spaces, algebras of general convexity and general concavity, General convex functional analysis, physics of general convexity and general concavity, Einstein's physics, Nonlinear programming, principles of relativity, Inequalities of transversal general convex functions. 


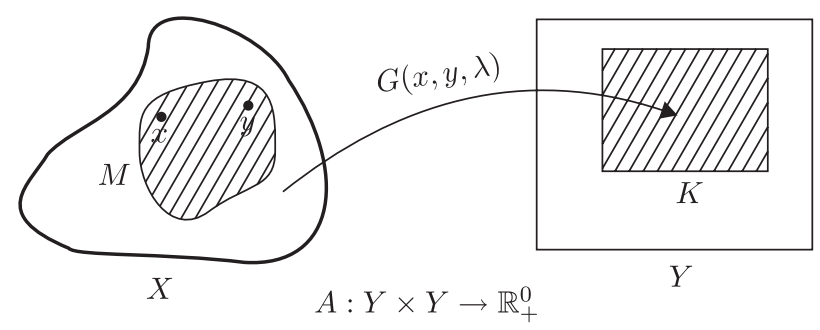

FiguRE 1.

A subset $K$ of $Y$ is transversal general convex if there is a subset $M$ of $X$ such that $G(x, y, \lambda) \in K$ for all $x, y \in M$. In this sense we say that $M$ and $K$ are transversal general convex subsets of $X$ and $Y$ respectively. Also, in this sense we say that $K$ (or $M$ ) is a transversal general convex set. If $X=Y$ and $M=K$ we say that $K$ is a general convex subset of $Y$.

In the special case, if $Y$ is a linear space we obtain a general convex linear space with the all characteristic properties of a linear space. In this sense in further we formulated a characteristic algebra with the elements of general convex structures.

We begin by restating the definition in terms of concepts now avaliable to us. The we will recall that by scalars we mean either the system of real numbers $\mathbb{R}$ or the system of complex numbers $\mathbb{C}$. A general convex linear space (or general convex vector space) is an additive Abelian group $L$ (whose elements are called general convex structures, see Fig. 2) with the property that any scalar $\alpha$ and any general convex structure $G(x, x, \lambda)$ can be combined by an operation called scalar multiplication to yield a general convex structure $\alpha G(x, x, \lambda)$ in such a manner that: $\alpha(G(x, x, \lambda)+$ $G(y, y, \lambda))=\alpha G(x, x, \lambda)+\alpha G(y, y, \lambda),(\alpha+\beta) G(x, x, \lambda)=\alpha G(x, x, \lambda)+$ $\beta G(x, x, \lambda),(\alpha \beta) G(x, x, \lambda)=\alpha(\beta G(x, x, \lambda))$, and $1 \cdot G(x, x, \lambda)=G(x, x, \lambda)$.

A general convex linear space is thus an additive Abelian group whose elements can be multiplied by numbers in a reasonable way, but not necessarily by one another (as in the case of ring). The two primary operations in a general convex linear space - addition and scalar multiplication - are called the general convex linear operations, and its zero element is usually referred to as the origin.

A general convex linear space is called a real general convex linear space or a complex general convex linear space according as the scalars are the real numbers or the complex numbers.

In further, a nonempty subset $S$ of $L$ is called a subspace (or a general convex linear subspace) of $L$ if $S$ is a general convex linear space in its own right with respect to the general convex linear operations defined in $L$. This is clearly equivalent to the condition that $S$ contain all sums, negatives, and scalar multiples of its elements; and since $-G(x, x, \lambda)=(-1) G(x, x, \lambda)$, this 

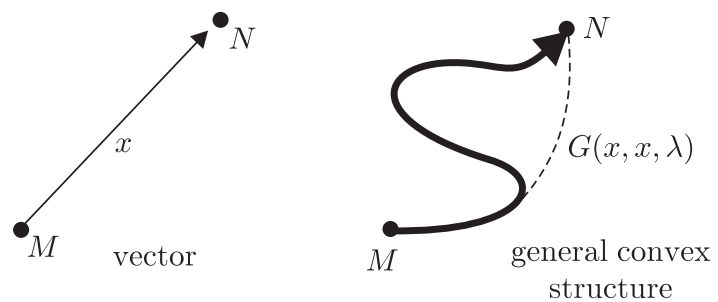

FiguRE 2.

in turn is equivalent to the condition that $S$ be closed under addition and scalar multiplication.

If the subspace $S$ is a proper subset of $L$, then it is called a proper subspace of $L$. The zero general convex linear space $\{0\}$ and the full space $L$ itself are always subspaces of $L$.

If $M$ is a general convex subspace of $L$, then - just as in the case of an ideal in a ring - we can use $M$ to define an equivalence relation in $L$ as follows: $G(x, x, \lambda) \equiv G(y, y, \lambda)(\bmod M)$ means that $G(x, x, \lambda)-G(y, y, \lambda)$ is in $M$. The discussion leading up to the preceding facts can be repeated without essential change (but with cosiderable simplification) to yield the concept of the quotient general convex linear space $L / M$ of $L$ with respect to $M$. We give a formal statement of the basic facts in the following statement.

Theorem 1. Let $M$ be a subspace of a general convex linear space $L$, and let the coset of an element $G(x, x, \lambda)$ in $L$ be defined by $G(x, x, \lambda)+M=$ $\{G(x, x, \lambda)+m: m \in M\}$. Then the distinct cosets form a partition of $L$; and if addition and scalar multiplication are defined by

$$
(G(x, x, \lambda)+M)+(G(y, y, \lambda)+M)=(G(x, x, \lambda)+G(y, y, \lambda))+M
$$

and $\alpha(G(x, x, \lambda)+M)=\alpha G(x, x, \lambda)+M$, then these cosets constitute $a$ general convex linear space denoted by $L / M$ and called the quotient general convex linear space of $L$ with respect to $M$. The origin in $L / M$ is the coset $0+M=M$, and the negative of $G(x, x, \lambda)+M$ is $(-G(x, x, \lambda))+M$.

The proof of this statement is routine, and we leave the details to the reader. We notice that the concept of a quotient general convex linear space has a simple geometric interpretation.

The subspaces of our general convex linear space $L$ can be characterized conveniently as follows. If $\left\{G\left(x_{1}, x_{1}, \lambda\right), \ldots, G\left(x_{n}, x_{n}, \lambda\right)\right\}$ is a finite nonempty set of elements in $L$, then the element of the form as

$$
x=\alpha_{1} G\left(x_{1}, x_{1}, \lambda\right)+\cdots+\alpha_{n} G\left(x_{n}, x_{n}, \lambda\right)
$$

is called a general convex linear combination of $G\left(x_{1}, x_{1}, \lambda\right), \ldots, G\left(x_{n}\right.$, $\left.x_{n}, \lambda\right)$. It is evident that a subspace of $L$ is simply a nonempty subset of $L$ which is closed under the formation of general convex linear combination. 
(Otherwise, if $\left\{G\left(x_{1}, x_{2}, \lambda\right), \ldots, G\left(x_{n-1}, x_{n}, \lambda\right), G\left(x_{n}, x_{1}, \lambda\right)\right\}$ is a finite nonempty set of elements in $L$, then the element of the form as

$$
x=\alpha_{1} G\left(x_{1}, x_{2}, \lambda\right)+\cdots+\alpha_{n-1} G\left(x_{n-1}, x_{n}, \lambda\right)+\alpha_{n} G\left(x_{n}, x_{1}, \lambda\right)
$$

can be is called a general convex linear combination of $G\left(x_{1}, x_{2}, \lambda\right), \ldots, G\left(x_{n-1}, x_{n}, \lambda\right)$, $G\left(x_{n}, x_{1}, \lambda\right)$. Also, it is evident that a subspace of $L$ is simply a nonempty subset of $L$ which is closed under the formation of general convex linear combination. Only from technical reason in further we to work with definition (1). All facts also hold and for definition (2)).

If $S$ is an arbitrary nonempty subset of $L$, then the set of all general convex linear combination of elements in $S$ is clearly a subspace of $L$; we denote this subspace by $[\mathrm{S}]$, and we call it the subspace spanned by $S$. Since [S] is a subspace which contains $S$ and is contained in every subspace which contains $S$, we my think of [S] as the smallest subspace which contains $S$. If $M$ is a subspace of $L$, then a nonempty subset $S$ of $M$ is said to span $M$ if $[S]=M$.

Suppose now that $M$ and $N$ are subspaces of $L$, and consider the set $M+N$ of all sums of the form $G(x, x, \lambda)+G(y, y, \lambda)$, where $G(x, x, \lambda) \in M$ and $G(y, y, \lambda) \in N$. Since $M$ and $N$ are subspaces, it is easy to see that $M+N$ is the subspace spanned by all elements in $M$ and $N$ together, i.e., that $M+N=[M \cup N]$. If it happens that $M+N=L$, then we say that $L$ is the sum of the subspaces $M$ and $N$. This means that each element in $L$ is expressible as the sum of an element in $M$ and an element in $N$.

The case in which even more is true - namely, that each element $z \in$ $L$ is expressible uniquely in the form $z=G(x, x, \lambda)+G(y, y, \lambda)$, with $G(x, x, \lambda) \in M$ and $G(y, y, \lambda) \in N$ - will be of particular importance for us. In this case we say that $L$ is the general convex direct sum (or only direct sum) of the subspaces $M$ and $N$, and we symbolize this statement by writing $L=M \oplus N$.

Theorem 2. Let a general convex linear space $L$ be the sum of two subspaces $M$ and $N$, so that $L=M+N$. Then $L=M \oplus N$ if and only if $M \cap N=\{0\}$.

The proof of this tatement is routine, and we leave the details to the reader. (The concept of a general convex direct sum can easily be broadened to allow for three or more subspaces.)

The dimension of a general convex linear space. Let $L$ be a general convex linear space, and let $S=\left\{G\left(x_{1}, x_{1}, \lambda\right), \ldots, G\left(x_{n}, x_{n}, \lambda\right)\right\}$ be a finite nonempty set of elements in $L$. The set $S$ is said to be linearly dependent (or general convex linearly dependent) if there exist scalars $\alpha_{1}, \alpha_{2}, \ldots, \alpha_{n}$ not all of which are 0 , such that

$$
\alpha_{1} G\left(x_{1}, x_{1}, \lambda\right)+\alpha_{2} G\left(x_{2}, x_{2}, \lambda\right)+\cdots+\alpha_{n} G\left(x_{n}, x_{n}, \lambda\right)=0 .
$$

If $S$ is not linearly dependent, then it is called linearly independent (or general convex linearly independent); and this clearly means that if the 
equation (3) holds for certain scalar coefficients $\alpha_{1}, \alpha_{2}, \ldots, \alpha_{n}$ then all these scalars are necessarily 0 . In either case, as we know, the elements in the subspace $[S]$ spanned by $S$ are precisely the general convex linear combinations

$$
x=\alpha_{1} G\left(x_{1}, x_{1}, \lambda\right)+\alpha_{2} G\left(x_{2}, x_{2}, \lambda\right)+\cdots+\alpha_{n} G\left(x_{n}, x_{n}, \lambda\right)
$$

of the $x_{i}{ }^{\prime} s$. The significance of the general convex linear independence of $S$ rests on the fact that if $S$ is linarly independent, then each element $x \in[S]$ is uniquely expressible in this form.

It is necessary to extend these concepts to cover the case of an arbitrary nonempty set of elements in $L$. We shall say that such a set is linearly independent (or general convex linearly independent) if every finite nonempty subset is general convex linearly independent in the sense of the above paragraph; otherwise, it is said to be linearly dependent (or general convex linearly dependent).

Just as in the finite case, an arbitrary nonempty subset $S$ of $L$ is linearly independent if and only if each element in the subspace $[S]$ spanned by $S$ is uniquely expressible as a general convex linearly combination of the elements in $S$.

We are particularly interested in linearly independent sets which span the whole space $L$. Such a set is called a general convex basis (or only basis) for $L$. It is important to observe that if $S$ is a general convex linearly independent subset of $L$, then $S$ is a basis for $L$ if and only if it is maximal (or general convex maximal) with respect to being general convex linearly independent, in the sense that every subset of $L$ which properly contains $S$ is general convex linearly dependent.

Our first statement assures us that if a general convex linearly independent set is not already a basis, then it can always be enlarged to form a basis.

Theorem 3. If $S$ is a general convex linearly independent set of elements in a general convex linear space $L$, then there exists a general convex basis $B$ for $L$ such that $S \subset B$.

This statement is a consequence of Zorn's lemma. The proof of this statement is routine, and we leave the details to the reader.

Theorem 4. Let $S=\left\{G\left(x_{1}, x_{1}, \lambda\right), \ldots, G\left(x_{n}, x_{n}, \lambda\right)\right\}$ be a finite nonempty set of elements in a general convex linear space $L$. If $n=1$, then $S$ is linearly dependent if and only if $G\left(x_{1}, x_{1}, \lambda\right)=0$. If $n>1$ and $G\left(x_{1}, x_{1}, \lambda\right) \neq$ 0 , then $S$ is linearly dependent if and only if some one of the elements $G\left(x_{2}, x_{2}, \lambda\right), \ldots, G\left(x_{n}, x_{n}, \lambda\right)$ is a general convex linear combination of the elements in $S$ which precede it. 
On the other hand, if $L \neq\{0\}$ and $G(x, x, \lambda)$ is a nonzero element in $L$, then the single element set $\{G(x, x, \lambda)\}$ is general convex linearly independent and Theorem 3 guarantees that $L$ has a general convex basis which contains $\{G(x, x, \lambda)\}$. This proves the following result.

Theorem 5. Every nonzero general convex linear space has a general convex basis. (This statement is also a consequenca of Zorn's lemma.)

We are now in a position to formulation our main result (as and a restricted form) in its full generality in the form as.

Theorem 6. Let $L$ be a nonzero general convex linear space. If $B_{1}=$ $\left\{G\left(e_{i}, e_{i}, \lambda\right)\right\}$ and $B_{2}=\left\{G\left(f_{i}, f_{i}, \lambda\right)\right\}$ are any two bases for $L$, then $B_{1}$ and $B_{2}$ have the same number of elements; that is, the same cardinal number. (If $L$ has a finite basis $B_{1}=\left\{G\left(e_{1}, e_{1}, \lambda\right), \ldots, G\left(e_{n}, e_{n}, \lambda\right)\right\}$ with $n$ elements, then any other basis $B_{2}$ is also finite and also has $n$ elements.)

For the second part of statement, to show that $B_{2}$ is finite, we assume that it is not, and we deduce a contradiction from this assumption. The proof of main first part of statement is routine.

In further, let $L$ and $L^{\prime}$ be general convex linear spaces with the same system of scalars. An isomorphism of $L$ onto $L^{\prime}$ is a one-to-one mapping $f$ of $L$ onto $L^{\prime}$ such that

$$
f(G(x, x, \lambda)+G(y, y, \lambda))=f(G(x, x, \lambda))+f(G(y, y, \lambda))
$$

and $f(\alpha G(x, x, \lambda))=\alpha f(G(x, x, \lambda))$; and if there exists such an isomorphism, then $L$ is said to be isomorphic to $L^{\prime}$. To say that one general convex linear space is isomorphic to another is to say, in effect, that they are abstractly identical with respect to their structure as general convex linear spaces.

Theorem 7. Let $L$ be a nonzero finite dimensional general convex linear space of dimension $m$. If $L$ is real, then it is isomorphic to $\mathbb{R}^{m}$; and if it is complex, then it is isomorphic to $\mathbb{C}^{m}$.

This statement can easily to extended to the case of an arbitrary nonzero general convex linear space. In this sense, from the preceding facts, the corresponding discussion yields the following adequate result.

Theorem 8. Let $L$ be a nonzero general convex linear space. If $B$ is a basis for $L$, then $L$ isomorphic to the general convex linear space $L(B)$ of all scallars valued functions defined on $B$ which vanish outside finite sets.

The preceding statements are of considerable interest in that they reveal what simple things general convex linear spaces really are. Otherwise, a reason is that almost all the general convex linear spaces of greatest interest carry additional algebraic or topological structure, which need not be related in any significant manner to the above isomorphisms. 
General convex linear transformations. Let $L$ and $L^{\prime}$ be general convex linear spaces with the same system of scalars. A mapping $T$ of $L$ into $L^{\prime}$ is called a general convex linear transformation if

$$
T(G(x, x, \lambda)+G(y, y, \lambda))=T(G(x, x, \lambda))+T(G(y, y, \lambda))
$$

and $T(\alpha G(x, x, \lambda))=\alpha T(G(x, x, \lambda))$ or equivalently, if the following equality holds in the corresponding adequate form as

$$
T(\alpha G(x, x, \lambda)+\beta G(y, y, \lambda))=\alpha T(G(x, x, \lambda))+\beta T(G(y, y, \lambda)) .
$$

A general convex linear transformation of one general convex linear space into another is thus a homomorphism of the first space into the second, for it is a mapping which preserves the general convex linear operations. Also, $T$ preserves the origin an negatives, for $T(0)=T(0 \cdot 0)=0 \cdot T(0)=0$ and

$$
T(-G(x, x, \lambda))=T((-1) G(x, x, \lambda))=(-1) T(G(x, x, \lambda))=-T(G(x, x, \lambda)) .
$$

The importance of general convex linear spaces lies mainly in the general convex linear transformations they carry, for vast tracts of algebra and analysis, when placed in their proper context, reduce to the study of general convex linear transformations of one general convex linear space into another.

The theory of matrices, for instance, is one small corner of this subject, as are the theory of certain types of differential and integral equations and the theory of integration in its most elegant modern form.

Algebras. A general convex linear space $\mathcal{A}$ is called an algebra (or general convex algebra) if its elements can be multiplied in such a way that $\mathcal{A}$ is also a ring in which scalar multiplication is related to multiplication by the following property: $\alpha(G(x, x, \lambda) G(y, y, \lambda))=(\alpha G(x, x, \lambda)) G(y, y, \lambda)=$ $G(x, x, \lambda)(\alpha G(y, y, \lambda))$.

The concept of an algebra is therefore a natural combination of the concepts of a general convex linear space and a ring. On Figure 3 illustrates the manner in which the major algebraic systems defined in this chapter are related to one another.

In the next, a division algebra is an algebra with identity which, as a ring, is a division ring. A subalgebra of an algebra $\mathcal{A}$ is a nonempty subset $\mathcal{A}_{0}$ of $\mathcal{A}$ which is an algebra in its own right with respect to the operations in $\mathcal{A}$. This condition evidently means that $\mathcal{A}_{0}$ is closed under addition, scalar multiplication, and multiplication.

An ideal $I d$ in algebra $\mathcal{A}$ is a nonempty subset of $\mathcal{A}$ which is both a subspace when $\mathcal{A}$ is considered as a general convex linear space and an ideal when $\mathcal{A}$ is considered as a ring. It is easy to see that $\mathcal{A} / I d$ is actually an algebra, called the quotient algebra (or general convex quotient algebra) of $\mathcal{A}$ with respect to $I d$. An ideal in $\mathcal{A}$ in our present sense is sometimes called an algebra ideal, as opposed to what we might call a ring ideal, that is, an ideal in $\mathcal{A}$ when $\mathcal{A}$ is considered as a ring. By our definition, an algebra ideal is a ring ideal which is also a subspace. 


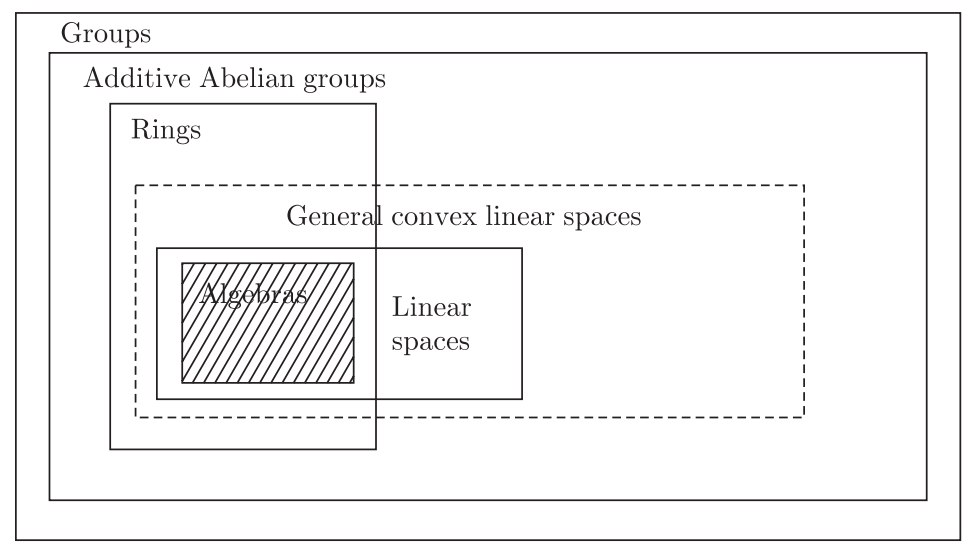

Figure 3. (The major algebraic systems)

In the next we have a combination of algebraic and geometric structures opens up the possibility of studying general convex linear transformations of one general convex linear space into another which have the additional property of being continuous.

In this sense, a normed general convex linear space is a general convex linear space $X$ in which to each element $G(x, x, \lambda)$ there corresponds a real number, denoted by $\|G(x, x, \lambda)\|$ and called the norm (or general convex norm) of $G(x, x, \lambda)$, in such a manner that: $\|G(x, x, \lambda)\| \geq 0$, $\|G(x, x, \lambda)\|=0$ if and only if $G(x, x, \lambda)=0,\|\alpha G(x, x, \lambda)\|=|\alpha|\|G(x, x, \lambda)\|$, and if there exists a function $g:\left(\mathbb{R}_{+}\right)^{2} \rightarrow \mathbb{R}_{+}^{0}$ such that

$\|G(x, y, \lambda)\| \leq \max \{\|G(x, x, \lambda)\|,\|G(y, y, \lambda)\|, g(\|G(x, x, \lambda)\|,\|G(y, y, \lambda)\|)\}$.

The nonegative real number $\|G(x, x, \lambda)\|$ is to be thought of as the length of the element $G(x, x, \lambda)$. If we regard $\|G(x, x, \lambda)\|$ as a real function defined on $X$, this function is called the norm (or general convex norm) on $X$. In the case $G(x, y, \lambda)=G(x, x, \lambda)+G(y, y, \lambda)$ and $g(s, t):=s+t$ we obtain an example of convex normed linear space $X$ where

$$
\|G(x, y, \lambda)\| \leq\|G(x, x, \lambda)\|+\|G(y, y, \lambda)\|,
$$

and where will find that the deeper statements, in which completeness hypotheses are necessary, often make essential use of Baire's theorem.

In this case, several simple but important facts about a normed convex linear space are based on the following inequality in the form as

$$
|\|G(x, x, \lambda)\|-\|G(y, y, \lambda)\|| \leq\|G(x, x, \lambda)-G(y, y, \lambda)\| .
$$

The main conclusion we draw from (4) is that the norm, i.e., convex norm, is a continuous function. Our first statement exhibits one of the most useful ways of forming new normed general convex linear spaces out of old ones. 
Theorem 9. Let $M$ be a closed general convex linear subspace of a normed general convex linear space $X$. If the norm of a coset $G(x, x, \lambda)+M$ in the quotient space $X / M$ is defined by

$$
\|G(x, x, \lambda)+M\|=\inf \{\|G(x, x, \lambda)+m\|: m \in M\},
$$

then $X / M$ is a normed general convex linear space. Further, if $X$ is a complete space, then so is $X / M$.

In further, let $N$ and $N^{\prime}$ be normed general convex linear spaces with the same scalars, and let $T$ be a general convex linear transformation of $N$ into $N^{\prime}$. When we say that $T$ is continuous, we mean that this amounts to the condition that $G\left(x_{n}, x_{n}, \lambda\right) \rightarrow G(x, x, \lambda)$ in $N$ implies that $T\left(G\left(x_{n}, x_{n}, \lambda\right)\right) \rightarrow$ $T(G(x, x, \lambda))$ in $N^{\prime}$.

Theorem 10. Let $N$ and $N^{\prime}$ be normed general convex linear spaces and $T$ a linear convex transformation of $N$ into $N^{\prime}$. Then the following conditions on $T$ are all equivalent to one another: (a) $T$ is continuous; (b) $T$ is continuous at the origin, in the sense that $G\left(x_{n}, x_{n}, \lambda\right) \rightarrow 0$ implies that $T\left(G\left(x_{n}, x_{n}, \lambda\right)\right) \rightarrow 0$; (c) there exists a real number $K \geq 0$ with the property that

$$
\|T(G(x, x, \lambda))\| \leq K\|G(x, x, \lambda)\| \quad \text { for all } \quad G \in N ;
$$

and if $S:=\{G(x, x, \lambda):\|G(x, x, \lambda)\| \leq 1\}$ is the closed unit sphere in $N$, then its image $T(S)$ is a bounded set in $N^{\prime}$.

If the linear general convex transformation $T$ satisfies the following condition so that there exists a real number $K \geq 0$ with the property that (5), then $K$ is called a bound for $T$, and such a $T$ is often referred to as bounded general convex linear transformation. In this case we assume that $T$ is continuous and we define its norm by

$$
\|T\|:=\sup \{\|T(G(x, x, \lambda))\|:\|G(x, x, \lambda)\| \leq 1\} .
$$

We notice that when $N \neq\{0\}$, this formula (i.e., this equality) can clearly be written in the following equivalent form as

$$
\|T\|=\sup \{\| T(G(x, x, \lambda)))\|:\| G(x, x, \lambda) \|=1\} .
$$

It is apparent from the preceding facts that the set of all bounds for $T$ equals the set of all radii of closed spheres centered on the origin which contain $T(S)$. This yields yet another expression for the norm of $T$ namely,

$$
\|T\|=\inf \{K \geq 0:\|T(G(x, x, \lambda))\| \leq K\|G(x, x, \lambda)\| \text { for all } x\} ;
$$

and hence from this we see at once that $\|T(G(x, x, \lambda))\| \leq\|T\|\|G(x, x, \lambda)\|$ for all $G \in N$ and for all $x \in X$.

We denote the set of all continuous (or bounded) general convex linear transformations of $N$ into $N^{\prime}$ by $\mathcal{B}\left(N, N^{\prime}\right)$. The following statement is in further applicable. 
Theorem 11. If $N$ and $N^{\prime}$ are normed general convex linear spaces, then the set $\mathcal{B}\left(N, N^{\prime}\right)$ is a normed general convex linear space with respect to the pointwise linear general convex linear operations and the norm defined by (6). Further, if $N^{\prime}$ is a complete space, then $\mathcal{B}\left(N, N^{\prime}\right)$ is also a complete space.

We complete this section with some definitions which will often be useful in our later work. Let $N$ and $N^{\prime}$ be normed general convex linear spaces. An isometric isomorphism of $N$ into $N^{\prime}$ is a one-to-one general convex linear transformation $T$ of $N$ into $N^{\prime}$ such that

$$
\|T(G(x, x, \lambda))\|=\|G(x, x, \lambda)\| \quad \text { for all } G \in N ;
$$

and $N$ is said to be isometrically isomorphic to $N^{\prime}$ if there exists an isometric isomorphism of $N$ onto $N^{\prime}$. This terminology enables us to give precise meaning to the statement that one normed general convex linear space is essentially the same as another.

Extension of Hahn-Banach theorem. One of the basic principles of strategy in the study of an abstract mathematical system can be stated as follows: consider the set of all structure-preserving mappings of that system into the simplest system of the same type. This principle is richly fruitful in the structure theory (or representation theory) of groups, rings, and algebras, and we shall see in the next how it works for normed general convex linear spaces.

We have remarked that the spaces $\mathbb{R}$ and $\mathbb{C}$ are the simplest of all normed general convex linear spaces. If $N$ is an arbitrary normed general convex linear space, the above principle leads us to form the set of all continuous general convex linear transformations of $N$ into $\mathbb{R}$ or $\mathbb{C}$, according as $N$ is real or complex. This set-it is $\mathcal{B}(N, \mathbb{R})$ or $\mathcal{B}(N, \mathbb{C})$ - is denoted by $N^{*}$ and is called the conjugate space of $N$. The elements of $N^{*}$ are called continuous general convex linear functionals, or only functionals.

Most of the theory of conjugate spaces rests on a form of Hahn-Banach theorem, which asserts that any functional, defined on a linear subspace of a normed general convex linear space, can be extended linearly and continuously to the whole space without increasing its norm. The proof is rather complicated, so we begin with a statement which serves to isolate its most difficult parts.

Proposition 1. Let $M$ be a linear subspace of a normed general convex linear space $N$, and let $f$ be a functional defined on $M$. If $G\left(x_{0}, x_{0}, \lambda\right)$ is an element not in $M$, and if $M_{0}=M+\left[G\left(x_{0}, x_{0}, \lambda\right)\right]$ is the linear subspace spanned by $M$ and $G\left(x_{0}, x_{0}, \lambda\right)$, then $f$ can be extended to a functional $f_{0}$ defined on $M_{0}$ such that $\left\|f_{0}\right\|=\|f\|$.

We first prove this statement under the assumption that $N$ is a real normed general convex linear space. We next use the result of the above 
facts to prove the statement for the case in which $N$ is complex. The following result is a form of Hahn-Banach theorem in the following form as.

Theorem 12. Let $M$ be a linear subspace of a normed general convex linear space $N$, and let $f$ be a functional defined on $M$. Then $f$ can be extended to a functional $f_{0}$ defined on the whole space $N$ such that $\left\|f_{0}\right\|=\|f\|$.

Proof. The set of all extensions of $f$ to functionals $h$ with the same norm defined on subspaces which contain $M$ is clearly a partially ordered set with respect to the following relation: $h_{1} \preccurlyeq h_{2}$ means that the domain of $h_{1}$ is contained in the domain of $h_{2}$, and $h_{2}(x)=h_{1}(x)$ for all $x$ in the domain of $h_{1}$. It is easy to see that the union of any chain of extensions is also an extension and is therefore an upper bound for the chain. Zorn's lemma now implies that there exists a maximal extension $f_{0}$. We complete the proof by observing that the domain of $f_{0}$ must be the entire space $N$, for otherwise it could be extended further by our Proposition 1 and would not be maximal.

In the preceding sense, the property that every complete general convex linear space has a rich supply of functionals is to be understood in the sense of the following two statements, on which most of its applications depend.

Theorem 13. If $N$ is a general convex linear space and $G\left(x_{0}, x_{0}, \lambda\right)$ is a nonzero element in $N$, then there exists a functional $f_{0}$ in $N^{*}$ such that $f_{0}\left(G\left(x_{0}, x_{0}, \lambda\right)\right)=\left\|G\left(x_{0}, x_{0}, \lambda\right)\right\|$ and $\left\|f_{0}\right\|=1$.

Among other things, this result shows that $N^{*}$ separates the elements in $N$, for if $G(x, x, \lambda)$ and $G(y, y, \lambda)$ are any two distinct elements, so that $G(x, x, \lambda)-G(y, y, \lambda) \neq 0$, then there exists a functional $f$ in $N^{*}$ such that $f(G(x, x, \lambda)-G(y, y, \lambda)) \neq 0$, or equivalently, $f(G(x, x, \lambda)) \neq f(G(y, y, \lambda))$.

Theorem 14. If $M$ is a closed linear subspace of a normed general convex linear space $N$ and $G\left(x_{0}, x_{0}, \lambda\right)$ is an element not in $M$, then there exists a functional $f_{0}$ in $N^{*}$ such that $f_{0}(M)=0$ and $f_{0}\left(G\left(x_{0}, x_{0}, \lambda\right)\right) \neq 0$.

These statements play a critical role in the ideas developed in the following parts, and their significance will emerge quite clearly in the proper context.

The natural imbedding of $N$ in $N^{* *}$. Since the conjugate space $N^{*}$ of a normed general convex linear space $N$ is itself a normed general convex linear space, it is possible to form the conjugate space $\left(N^{*}\right)^{*}$ of $N^{*}$. We denote this space by $N^{* *}$, and we call it the second conjugate space of $N$.

The importance of $N^{* *}$ rests on the fact that each element $G(x, x, \lambda)$ in $N$ gives rise to a functional $F_{x}$ in $N^{* *}$. If we denote a typical element of $N^{*}$ by $f$, then $F_{x}$ is defined by $F_{x}(f)=f(x)$. A simple manipulation of the definition shows that $F_{x}$ is linear and $\left\|F_{x}\right\| \leq\|G(x, x, \lambda)\|$, i.e., from Theorem 13 is exactly what is needed to guarantee that equality holds here, 
so far each $G(x, x, \lambda)$ in $N$ we have

$$
\left\|F_{x}\right\|=\|G(x, x, \lambda)\| .
$$

It follows from these observations that $x \mapsto F_{x}$ is a norm-preserving mapping of $N$ into $N^{* *} . F_{x}$ is called the functional on $N^{*}$ induced by the element $G(x, x, \lambda)$, and we refer to functionals of this kind as induced functionals.

The isometric isomorphism $x \mapsto F_{x}$ is called the natural imbedding of $N$ in $N^{* *}$, for it allows us to regard $N$ as part of $N^{* *}$ without altering any of its structure as normed general convex linear space. We write $N \subset N^{* *}$, where this set inclusion is to be understood in the sense just explained.

Theorem 15. If $N$ is a normed general convex linear space, then the closed unit sphere $S^{*}$ in $N^{*}$ is a compact Hausdorff space in the weak* topology.

We are now in a position to keep the promise made in the last part of this section for the following result is an obvious consequence of our preceding work.

Theorem 16. Let $N$ be a normed general convex linear space, and let $S^{*}$ be the compact Hausdorff space obtained by imposing the weak* topology on the closed unit sphere in $N^{*}$. Then the mapping $x \mapsto F_{x}$, where $F_{x}(f)=$ $f(G(x, x, \lambda))$ for each $f$ in $S^{*}$, is an isometric isomorphism of $N$ into $C\left(S^{*}\right)$. If $N$ is a complete space, this mapping is an isometric isomorphism of $N$ onto a closed linear subspace of $C\left(S^{*}\right)$.

Form of the open mapping theorem. In this part we have our first encounter with basic statements which require that the spaces concerned be complete. The following rather technical statement is the key to these statements.

Proposition 2. If $N$ and $N^{\prime}$ are complete normed general convex linear spaces, and if $T$ is a continuous general convex linear transformation of $N$ onto $N^{\prime}$, then the image of each open sphere centered on the origin in $N$ contains an open sphere centered on the origin in $N^{\prime}$.

We are now in a position that formulated the main statement in the preceding sense as a form of the open mapping theorem in the following form as.

Theorem 17. If $N$ and $N^{\prime}$ are complete normed general convex linear spaces, and if $T$ is a continuous general convex linear transformation of $N$ onto $N^{\prime}$, then $T$ is an open mapping.

Most of the applications of the form of the open mapping theorem depend more directly on the following special case, which we state separately for the sake of emphasis. 
Theorem 18. An one-to-one continuous general convex linear transformation of one complete normed general convex linear space onto another is a homeomorphism. In particular, if a one-to-one linear transformation $T$ of a complete normed general convex linear space onto itself is continuous, then its inverse $T^{-1}$ is automatically continuous.

As a first application of Theorem 18 we can give a geometric characterization of the projections on a complete normed general convex linear space.

Theorem 19. If $P$ is a projection on a complete normed general convex linear space $L$, and if $M$ and $N$ are its range and null space of $P$, then $M$ and $N$ are closed linear subspaces of $L$ such that $L=M \oplus N$.

We notice that a pair of linear subspaces $M$ and $N$ such that $L=M \oplus N$ determines a projection $E$ whose range and null space are $M$ and $N$ (if $z=G(x, x, \lambda)+G(y, y, \lambda)$ is the unique representation of an element in $L$ as a sum of elements in $M$ and $N$, then $E$ is defined by $E(z)=G(x, x, \lambda))$.

Theorem 20. Let $L$ be a complete normed general convex linear space, and let $M$ and $N$ be closed linear subspaces of $L$ such that $L=M \oplus N$. If $z=G(x, x, \lambda)+G(y, y, \lambda)$ is the unique representation of an element in $L$ as a sum of elements in $M$ and $N$, then the mapping $P$ defined by $P(z)=G(x, x, \lambda)$ is a projection on $L$ whose range and null space are $M$ and $N$.

We now turn to form of closed graph theorem. Let $L$ and $L^{\prime}$ be complete normed general convex linear spaces. If we define a metric $\rho$ on the product $L \times L^{\prime}$ by the following equality in the form as

$$
\rho\left[\left(x_{1}, y_{1}\right),\left(x_{2}, y_{2}\right)\right]:=\max \left\{\left\|x_{1}-x_{2}\right\|,\left\|y_{1}-y_{2}\right\|\right\},
$$

then the resulting topology is easily seen to be the same as the product topology, and convergence with respect to this metric is equivalent to coordinatewise convergence.

Now let $T$ be a linear general convex transformation of $L$ into $L^{\prime}$. We recall that the graph of $T$ is that subset of $L \times L^{\prime}$ which consists of all ordered pairs of the form $(G(x, x, \lambda), T(G(x, x, \lambda)))$. We can shows that if $T$ is continuous, then its graph is closed as a subset of $L \times L^{\prime}$. In the present context, the converse is also true.

Theorem 21. (Form of the Closed Graph Theorem). If $L$ and $L^{\prime}$ are complete normed general convex linear spaces and if $T$ is a linear general convex transformation of $L$ into $L^{\prime}$, then $T$ is continuous if and only if its graph is closed.

This form of the closed graph theorem has a number of interesting applications to problems in analysis. 
Form of the Banach-Steinhaus theorem. We shall see in this part that each operator $T$ on a normed general convex linear space $N$ induces a corresponding operator, denoted by $T^{*}$ and called the conjugate of $T$, on the conjugate space $N^{*}$. Our first task is to define $T^{*}$, and our second is to investigate the properties of the mapping $T \rightarrow T^{*}$. We base our discussion on the following statement.

Theorem 22. (Form of the Uniform Boundedness theorem). Let $X$ be a nonempty set and let $L$ be a complete normed general convex linear space and $N$ a normed general convex linear space. If $\left\{T_{i}\right\}_{i \in I}$ is a nonempty set of continuous general convex linear transformations of $L$ into $N$ with the property that $\left\{T_{i}(z)\right\}_{i \in I}$ is a bounded subset of $N$ for each element $z:=$ $G(x, x, \lambda) \in L$ and for each $x \in X$, where $I$ is an index set, then $\left\{\left\|T_{i}\right\|\right\}_{i \in I}$ is a bounded set of numbers; that is, $\left\{T_{i}\right\}_{i \in I}$ is bounded as a subset of $\mathcal{B}(L, N)$.

In the special case if the general convex structure $G(x, x, \lambda)=x \in L$, then this statement is often called the Banach-Steinhaus theorem, and it has several significant applications to analysis. See, for example, Z y g m und [1959, pp. 165-168]. For the purposes we have in view, our main interest is in the following simple consequence of it.

Theorem 23. A nonempty subset $S$ of a normed general convex linear space $N$ is bounded if and only $f(S)$ is a bounded set of numbers for each $f \in N^{*}$.

Let $L$ be the general convex linear space of all scallar-valued linear functions defined on a normed general convex linear space $N$. The conjugate space $N^{*}$ is clearly a linear subspace of $L$. Let $T$ be a linear general convex transformation of $N$ into itself which is not necessarily continuous. We use $T$ to define a linear general convex transformation $T^{\prime}$ of $L$ into itself, as follows. If $f$ in $L$, then $T^{\prime}(f)$ is defined by

$$
\left[T^{\prime}(f)\right](x):=f(T(G(x, x, \lambda))) .
$$

We leave it to the reader to verify that $T^{\prime}(f)$ actually is linear as a function defined on $N$, and also that $T^{\prime}$ is linear as mapping of $L$ into itself.

We now assume that the linear general convex transformation $T$ is continuous and is therefore an operator on $N$. The preceding developments allow us to consider the restriction of $T^{\prime}$ to a mapping of $N^{*}$ into itself. We denote this restriction by $T^{*}$, and we call it the conjugate of $T$. The action of $T^{*}$ is given by

$$
\left[T^{*}(f)\right](x):=f(T(G(x, x, \lambda))),
$$

in which-in contrast to (7)- $f$ is understood to be a functional on $N$, and not merely a scalar-valued linear function. Hence, $T^{*}$ is clearly linear, and $\left\|T^{*}\right\| \leq\|T\|$ such that next computation $\left\|T^{*}\right\|=\|T\|$. The mapping $T \rightarrow$ $T^{*}$ is thus a norm-preserving mapping of $\mathcal{B}(N)$ into $\mathcal{B}\left(N^{*}\right)$. 
Theorem 24. If $T$ is an operator on a normed general convex linear space $N$, then its conjugate $T^{*}$ defined by (8) is an operator on $N^{*}$, and the mapping $T \rightarrow T^{*}$ is an isometric isomorphism of $\mathcal{B}(N)$ into $\mathcal{B}\left(N^{*}\right)$ which reverses products and preserves the identity transformation.

General convex scalar spaces. With the preceding ideas as a background, we are now in a position to give our basic definition.

A general convex scalar space $N$ is a complex complete normed general convex linear space whose norm arises from an inner product (or general convex inner product), that is, in which there is defined a complex function $(G(x, x, \lambda) \mid G(y, y, \lambda))$ of elements $G(x, x, \lambda)$ and $G(y, y, \lambda)$ for arbitraries $x, y \in X$ (= an arbitrary nonempty set) with the folowing properties:

$$
\begin{aligned}
(\alpha G(x, x, \lambda) \mid G(y, y, \lambda)) & =\alpha(G(x, x, \lambda) \mid G(y, y, \lambda)), \\
(G(x, x, \lambda)+G(y, y, \lambda) \mid G(z, z, \lambda)) & =(G(x, x, \lambda) \mid G(z, z, \lambda))+(G(y, y, \lambda) \mid G(z, z, \lambda)), \\
(G(x, x, \lambda) \mid G(x, x, \lambda)) & >0
\end{aligned}
$$

for $G(x, x, \lambda) \neq 0$, and if there exists a function $\psi: \mathbb{K} \times \mathbb{K} \rightarrow \mathbb{K}$ such that

$$
\begin{gathered}
|(G(x, x, \lambda) \mid G(y, y, \lambda))|^{2} \leq \max \{(G(x, x, \lambda) \mid G(x, x, \lambda)), \\
(G(y, y, \lambda) \mid G(y, y, \lambda)), \psi((G(x, x, \lambda) \mid(G(x, x, \lambda)),(G(y, y, \lambda) \mid G(y, y, \lambda))\}
\end{gathered}
$$

for all $G \in L$ and $x, y, z \in X$, and for every $\alpha \in \mathbb{K}$. It is evident that the following further relation in the form as

$$
(G(x, x, \lambda) \mid G(y, y, \lambda)+G(z, z, \lambda))=(G(x, x, \lambda) \mid G(y, y, \lambda))+(G(x, x, \lambda) \mid G(z, z, \lambda))
$$

is a direct consequence of the preceding facts of definition of on inner product. If the field $\mathbb{K}=\mathbb{R}$, then we have a real general convex scalar space.

Proposition 3. If $G(x, x, \lambda)$ and $G(y, y, \lambda)$ are any two elements in a general convex scalar space, then the following inequality holds in the form as

$$
|(G(x, x, \lambda) \mid G(y, y, \lambda))| \leq\|G(x, x, \lambda)\|\|G(y, y, \lambda)\| .
$$

The proof of this inequality is a totally analogy with the proof of well-known Schwarz inequality for Hilbert spaces. Also see: Ta s k o v i ć [22].

We notice that it follows easily from the inequality (10) that the inner product in a general convex scalar space is jointly continuous: $G\left(x_{n}, x_{n}, \lambda\right) \rightarrow G(x, x, \lambda)$ and $G\left(y_{n}, y_{n}, \lambda\right) \rightarrow G(y, y, \lambda)$ implies that $\left(G\left(x_{n}, x_{n}, \lambda\right) \mid G\left(y_{n}, y_{n}, \lambda\right)\right) \rightarrow(G(x, x, \lambda) \mid G(y$, $y, \lambda)$ ).

A well-known statement of elementary geometry states that the sum of the squares of the sides of a parallelogram equals the sum of the squares of its diagonals. This fact has an analogue in the present context, for in any general convex scalar space the so-called parallelogram law (or general convex parallelogram law) holds in the form as:

$$
\|G(x, x, \lambda)+G(y, y, \lambda)\|^{2}+\|G(x, x, \lambda)-G(y, y, \lambda)\|^{2}=2\|G(x, x, \lambda)\|^{2}+2\|G(y, y, \lambda)\|^{2} .
$$

This is readily proved by writing out the expression on the left in terms of inner products by Tasković [22].

The parallelogram law has the following important consequence for our further work in the next part of this paper. 
Proposition 4. A closed general convex subset $K$ of a general convex scalar space $L$ contains a unique element of smallest norm.

A proof of this statement may be found in: Task ovi ć [22]. This proof has be founded on the general convex parallelogram law.

An inspection of Fig. 4 will reveal the geometric motivation of (10) for computation of the form $\|G(x, x, \lambda)\|^{2}-|(G(x, x, \lambda) \mid G(y, y, \lambda))|^{2}$.

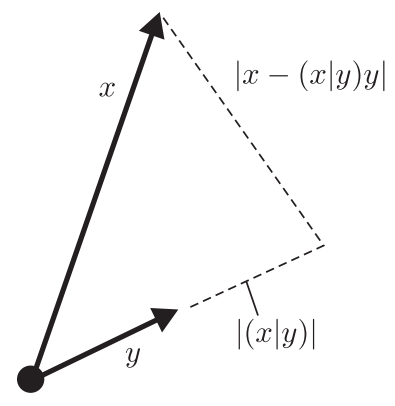

Schwarz's inequality

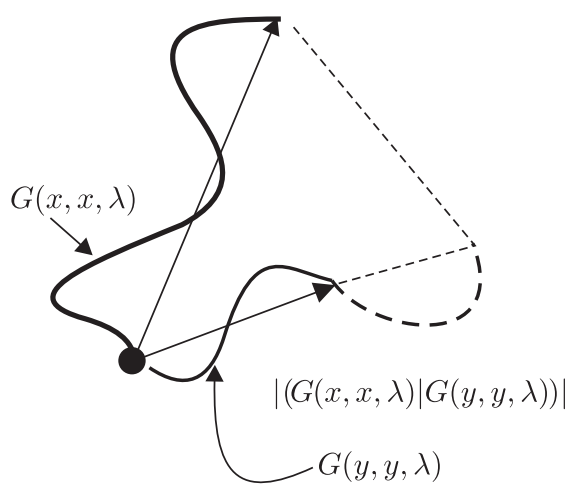

FigURE 4.

Let the general convex norm $\|G(x, x, \lambda)\|=\sqrt{(G(x, x, \lambda) \mid G(x, x, \lambda))}$ for an arbitrary $x \in X$, then we obrtain: $\|\alpha G(x, x, \lambda)\|=|\alpha|\|G(x, x, \lambda)\|$ and the characteristic inequality for the upper norm from (9). Thus a general convex scalar space is also and a transversal upper normed space. But, a first example of general convex scalar space is so-called inner-product space (i.e., over-Hilbert space).

There exist general convex scalar spaces (as and over-Hilbert spaces) which is not complete. For example, the space $C[a, b]$ with scalar product in the form

$$
(x \mid y):=\int_{a}^{b} x(t) \overline{y(t)} \mathrm{d} t
$$

is not complete. In this sense, let the sequence of functions $x_{k} \in C[a, b]$ for $k \in \mathbb{N}$ be a defined as on Fig. 5 by: $x_{k}(t)=0$ for $0 \leq t \leq c-1 / k, x_{k}(t)=1$ for $c+1 / k \leq t \leq b$, and $x_{k}(t)$ is an affine function on $[c-1 / k, c+1 / k]$.

As well-known, every over-Hilbert (or unitary) space is a normed space. When holds reverse fact of this, a solution was obtained in 1935 year by P. J o r d a n and J. von $\mathrm{Ne} \mathrm{u} \mathrm{m} \mathrm{a} \mathrm{n} \mathrm{n}$ in general case. Also see: S. K u r e p a [42], [43], and [44].

We notice that, in a complete general convex scalar space the following fact holds: if $\|G(x, x, \lambda)\|=\|G(y, y, \lambda)\|=1$ and if $\varepsilon>0$ is given, then there exists $\delta>0$ such that $\|(G(x, x, \lambda)+G(y, y, \lambda)) / 2\|>1-\delta$ implies that $\|G(x, x, \lambda)-G(y, y, \lambda)\|<\varepsilon$. A general convex space with this property is said to be uniformly convex (or uniformly general convex). See: Tasković [22].

The parallelogram law has another interesting application, which depends on the fact in any complete general convex scalar space the inner product is related 


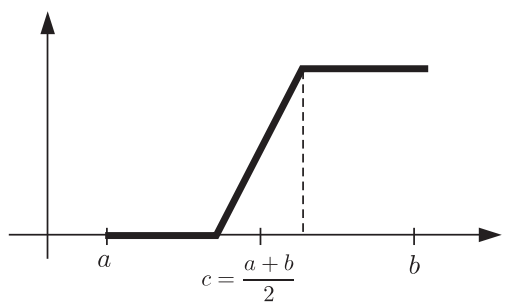

FiguRE 5.

to the upper norm by the following identity:

$$
\begin{gathered}
4(G(x, x, \lambda) \mid G(y, y, \lambda))= \\
=\|G(x, x, \lambda)+G(y, y, \lambda)\|^{2}-\|G(x, x, \lambda)-G(y, y, \lambda)\|^{2}+ \\
+i\|G(x, x, \lambda)+i G(y, y, \lambda)\|^{2}-i\|G(x, x, \lambda)-i G(y, y, \lambda)\|^{2} .
\end{gathered}
$$

This is easily verifed by converting on the right into general convex scalar product. In this sense the following statement holds.

Theorem 25. If $L$ is a complex complete normed general convex space whose norm obeys the parallelogram law and if a general convex scalar product is defined on $L$ by (11), then $L$ is a complete general convex scalar space.

This result has no implication at all for our future work. However, it does provide a satisfying geometric insight into the place general convex scalar spaces occupy among all complex complete general convex normed spaces: they are precisely those in which the parallelogram law is true.

Orthogonal complements. Two elements $G(x, x, \lambda)$ and $G(y, y, \lambda)$ in a complete general convex scalar space $L$ are said to be orthogonal (or general convex orthogonal) written $G(x, x, \lambda) \perp G(y, y, \lambda)$ if $(G(x, x, \lambda) \mid G(y, y, \lambda))=0$. The symbol $\perp$ is often pronounced "perp". If $\overline{(G(x, x, \lambda) \mid G(y, y, \lambda))}=(G(y, y, \lambda) \mid G(x, x, \lambda))$ we have $G(x, x, \lambda) \perp G(y, y, \lambda)$ if and only if $G(y, y, \lambda) \perp G(x, x, \lambda)$. It is also clear that $G(x, x, \lambda) \perp 0$ for every $G(x, x, \lambda)$ and for every $x \in X$, and $(G(x, x, \lambda) \perp G(x, x, \lambda))=$ $\|G(x, x, \lambda)\|^{2}$ shows that 0 is the only element orthogonal to itself. One of the simplest geometric facts about orthogonal elements is the Pythagorean theorem: if $G(x, x, \lambda) \perp G(y, y, \lambda)$ then the following equalities hold

$$
\begin{gathered}
\|G(x, x, \lambda)+G(y, y, \lambda)\|^{2}=\|G(x, x, \lambda)-G(y, y, \lambda)\|^{2}= \\
=\|G(x, x, \lambda)\|^{2}+\|G(y, y, \lambda)\|^{2} .
\end{gathered}
$$

An element $G(x, x, \lambda)$ is said to be orthogonal to a nonempty set $S$ (written $G(x, x, \lambda) \perp S)$ if $G(x, x, \lambda) \perp G(y, y, \lambda)$ for every $G(y, y, \lambda) \in S$ and for every $y \in X$, and the orthogonal complement of $S$ (denoted by $S^{\perp}$ ) is the set of all elements orthogonal to $S$.

Let $M$ be a closed linear subspace of a general convex scalar space $L$. We know that $M^{\perp}$ is also a closed linear subspace, and that $M$ and $M^{\perp}$ are disjoint in the sense that they have only the zero element in common. Our aim in this sense we have that $L=M \oplus M^{\perp}$, and each of our statements is a step in this direction. 
Theorem 26. Let $M$ be a closed linear subspace of a complete general convex scalar space $L$, let $G(x, x, \lambda)$ be an element not in $M$, and let $d$ be the distance from $G(x, x, \lambda)$ to $M$. Then there exists a unique element $G\left(x_{0}, x_{0}, \lambda\right) \in M$ for some $x_{0} \in X$ such that $\left\|G(x, x, \lambda)-G\left(x_{0}, x_{0}, \lambda\right)\right\|=d$. What else, if $M$ is a proper closed linear subspace of $L$, then there exists a nonzero element $G\left(z_{0}, z_{0}, \lambda\right)$ in $L$ such that $G\left(z_{0}, z_{0}, \lambda\right) \perp M$.

The proof of this statement may strike as being excessively dependent on ingenious computations. In this sense the following statement holds in the form as.

Theorem 27. If $M$ and $N$ are closed linear subspaces of a complete general convex scalar space $L$ such that $M \perp N$, then the linear subspace $M+N$ is also closed. Also, then $L=M \oplus M^{\perp}$.

The main effect of this statement is to guarantee that a complete general convex scalar space is always rich in projections. In fact, if $M$ is an arbitrary closed linear subspace of a complete general convex scalar space $L$, then Theorem 27 shows that there exists a projection defined on $L$ whose range is $M$ and whose null space is $M^{\perp}$.

Orthonormal sets. An orthonormal set (or general convex orthonormal set) in a complete general convex scalar space $S$ is a nonmepty subset of $S$ which consists of mutually orthogonal unit elements; that is, it is a nonempty subset $\left\{G\left(e_{i}, e_{i}, \lambda\right)\right\}$ of $S$ with the following properties: $i \neq j$ implies $G\left(e_{i}, e_{i}, \lambda\right) \perp G\left(e_{j}, e_{j}, \lambda\right)$ and $\left\|G\left(e_{i}, e_{i}, \lambda\right)\right\|=1$ for every $i \in I$ (= a set of of index).

If $S$ contains only the zero element, then it has no orthonormal sets. If $S$ contains a nonzero element $G(x, x, \lambda)$, and if we normalize $G(x, x, \lambda)$ by considering $G(e, e, \lambda)=G(x, x, \lambda) /\|G(x, x, \lambda)\|$, then the single element set $\{G(e, e, \lambda)\}$ is clearly an orthonormal set. More generaly, if $\left\{G\left(x_{i}, x_{i}, \lambda\right)\right\}$ is a nonempty set of mutually orthogonal nonzero elements in $S$, and if the $G\left(x_{i}, x_{i}, \lambda\right)$ 's are normalized by replacing each or them by $G\left(e_{i}, e_{i}, \lambda\right)=G\left(x_{i}, x_{i}, \lambda\right) /\left\|G\left(x_{i}, x_{i}, \lambda\right)\right\|$, then the resulting set $\left\{G\left(e_{i}, e_{i}, \lambda\right)\right\}$ is an orthonormal set.

Proposition 5. Let $\left\{G\left(e_{1}, e_{1}, \lambda\right), \ldots, G\left(e_{n}, e_{n}, \lambda\right)\right\}$ be a finite orthonormal set in a complete general convex scalar space $S$. If $G(x, x, \lambda)$ is any element in $S$, then the following inequality holds in the form as

$$
\sum_{i=1}^{n}\left|\left(G(x, x, \lambda) \mid G\left(e_{i}, e_{i}, \lambda\right)\right)\right|^{2} \leq\|G(x, x, \lambda)\|^{2} ;
$$

what else, in further context, the following fact holds in the form as

$$
G(x, x, \lambda)-\sum_{i=1}^{n}\left(G(x, x, \lambda) \mid G\left(e_{i}, e_{i}, \lambda\right)\right) G\left(e_{i}, e_{i}, \lambda\right) \perp G\left(e_{j}, e_{j}, \lambda\right)
$$

for every $G\left(e_{j}, e_{j}, \lambda\right)$ and for every $j \in I$, where $I$ is a nonempty index set. (This statement follows from Proposition 3.)

Our next task is to prove that both parts of Proposition 5 generalize to the case of an arbitrary orthonormal set. The key to this problem lies in the following statement. 
Proposition 6. If $\left\{G\left(e_{i}, e_{i}, \lambda\right)\right\}$ is an orthonormal set in a complete general convex scalar space $S$ and if $G(x, x, \lambda)$ is any element in $S$, then the set $O=\left\{G\left(e_{i}, e_{i}, \lambda\right)\right.$ : $\left.\left(G(x, x, \lambda) \mid G\left(e_{i}, e_{i}, \lambda\right)\right) \neq 0\right\}$ is either empty or countable.

As our first application of this result we can prove the general form of inequality (12) in the following form as.

Proposition 7. (Extension of inequality (12)). If $\left\{G\left(e_{i}, e_{i}, \lambda\right)\right\}$ is an orthonormal set in a complete general convex scalar space $S$, then the following inequality holds in the form as

$$
\sum_{i \in I}\left|\left(G(x, x, \lambda) \mid G\left(e_{i}, e_{i}, \lambda\right)\right)\right|^{2} \leq\|G(x, x, \lambda)\|^{2}
$$

for every element $G(x, x, \lambda)$ in $S$, for every $x \in X$, and for an arbitrary index set $I$. (The proof of this statement follows by Proposition 6.)

In connecation with this result as and with the second part of Proposition 6 we can give the following generalization in the form as.

Proposition 8. If $\left\{G\left(e_{i}, e_{i}, \lambda\right)\right\}$ is an orthonormal set in a complete general convex scalar space $S$ and if $G(x, x, \lambda)$ is an arbitrary element in $S$, then

$$
G(x, x, \lambda)-\sum_{i \in I}\left(G(x, x, \lambda) \mid G\left(e_{i}, e_{i}, \lambda\right)\right) G\left(e_{i}, e_{i}, \lambda\right) \perp G\left(e_{j}, e_{j}, \lambda\right)
$$

for every $j \in I$, where $I$ is an arbitrary index set. (The proof of this result follows from Proposition 6 and from inequality (13).)

Let $S$ be a nonzero complete general convex scalar space, so that the class of all its orthonormal sets is nonempty. This class is clearly a partially ordered set with respect to set inclusion. An orthonormal set $\left\{G\left(e_{i}, e_{i}, \lambda\right)\right\}$ in $S$ is said to be complete if it is maximal in this partially ordered set, that is, if it is impossible to adjoin an element $G(e, e, \lambda)$ to $\left\{G\left(e_{i}, e_{i}, \lambda\right)\right\}$ in such a way that $\left\{G\left(e_{i}, e_{i}, \lambda\right), G(e, e, \lambda)\right\}$ is an orthonormal set which properly contains $\left\{G\left(e_{i}, e_{i}, \lambda\right)\right\}$.

Proposition 9. Every nonzero complete general convex scalar space contains a complete orthonormal set.

Proof. The statement follows at once from Zorn's lemma, since the union of any chain of orthonormal sets is clearly an upper bound for the chain in the partially ordered set of all orthonormal sets. The proof is complete.

In connection with the preceding we notice that orthonormal sets are truly interesting only when thay are complete. The reasons for this are presented in our next statement.

Theorem 28. Let $S$ be a complete general convex scalar space and let $\left\{G\left(e_{i}\right.\right.$, $\left.\left.e_{i}, \lambda\right)\right\}$ be an orthonormal set in $S$. Then the following conditions are all equivalent to one another: $\left\{G\left(e_{i}, e_{i}, \lambda\right)\right\}$ is complete, $G(x, x, \lambda) \perp\left\{G\left(e_{i}, e_{i}, \lambda\right)\right\}$ implies that $G(x, x, \lambda)=0$, and if $G(x, x, \lambda)$ is an arbitrary element in $S$, then $G(x, x, \lambda)=$ $\sum\left(G(x, x, \lambda) \mid G\left(e_{i}, e_{i}, \lambda\right)\right) G\left(e_{i}, e_{i}, \lambda\right)$ and

$$
\|G(x, x, \lambda)\|^{2}=\sum\left|\left(G(x, x, \lambda) \mid G\left(e_{i}, e_{i}, \lambda\right)\right)\right|^{2} .
$$


An orthogonalization process. Suppose $\left\{G\left(x_{1}, x_{1}, \lambda\right), \ldots, G\left(x_{n}, x_{n}, \lambda\right), \ldots\right\}$ is a linearly independent set in a complete general convex scalar space $S$. The problem is to exhibit a constructive procedure for converting this set into a corresponding orthonormal set $\left\{G\left(e_{1}, e_{1}, \lambda\right), G\left(e_{2}, e_{2}, \lambda\right), \ldots, G\left(e_{n}, e_{n}, \lambda\right), \ldots\right\}$ with the property that for each $\mathrm{n}$ the linear subpsaces of $S$ spanned by $\left\{G\left(e_{1}, e_{1}, \lambda\right), \ldots\right.$, $\left.G\left(e_{n}, e_{n}, \lambda\right)\right\}$ in the same as that spanned by $\left\{G\left(x_{1}, x_{1}, \lambda\right), \ldots, G\left(x_{n}, x_{n}, \lambda\right)\right\}$. Our first step is to normalize $G\left(x_{1}, x_{1}, \lambda\right)$ - which is necessarily nonzero - by putting

$$
G\left(e_{1}, e_{1}, \lambda\right)=\frac{G\left(x_{1}, x_{1}, \lambda\right)}{\left\|G\left(x_{1}, x_{1}, \lambda\right)\right\|} .
$$

The next step is to subtract from $G\left(x_{2}, x_{2}, \lambda\right)$ its component in the direction of $G\left(e_{1}, e_{1}, \lambda\right)$ to obtain the element $G\left(x_{2}, x_{2}, \lambda\right)-\left(G\left(x_{2}, x_{2}, \lambda\right) \mid G\left(e_{1}, e_{1}, \lambda\right)\right) G\left(e_{1}, e_{1}, \lambda\right)$ orthogonal to $G\left(e_{1}, e_{1}, \lambda\right)$, and then to normalize this by putting

$$
G\left(e_{2}, e_{2}, \lambda\right)=\frac{G\left(x_{2}, x_{2}, \lambda\right)-\left(G\left(x_{2}, x_{2}, \lambda\right) \mid G\left(e_{1}, e_{1}, \lambda\right)\right) G\left(e_{1}, e_{1}, \lambda\right)}{\left\|G\left(x_{2}, x_{2}, \lambda\right)-\left(G\left(x_{2}, x_{2}, \lambda\right) \mid G\left(e_{1}, e_{1}, \lambda\right)\right) G\left(e_{1}, e_{1}, \lambda\right)\right\|} .
$$

We observe that since $G\left(x_{2}, x_{2}, \lambda\right)$ is not a scalar multiple of $G\left(x_{1}, x_{1}, \lambda\right)$, the element $G\left(x_{2}, x_{2}, \lambda\right)-\left(G\left(x_{2}, x_{2}, \lambda\right) \mid G\left(e_{1}, e_{1}, \lambda\right)\right) G\left(e_{1}, e_{1}, \lambda\right)$ is not zero, so the definition of $G\left(e_{2}, e_{2}, \lambda\right)$ is valid. Also, it is clear that $G\left(e_{2}, e_{2}, \lambda\right)$ is a linear combination $G\left(x_{1}, x_{1}, \lambda\right)$ and $G\left(x_{2}, x_{2}, \lambda\right)$, and that $G\left(x_{2}, x_{2}, \lambda\right)$ is a linear combination of $G\left(e_{1}, e_{1}, \lambda\right)$ and $G\left(e_{2}, e_{2}, \lambda\right)$.

The next step is to substract from $G\left(x_{3}, x_{3}, \lambda\right)$ its components in the directions of $G\left(e_{1}, e_{1}, \lambda\right)$ and $G\left(e_{2}, e_{2}, \lambda\right)$ to obtain an element orthogonal to $G\left(e_{1}, e_{1}, \lambda\right)$ and $G\left(e_{2}, e_{2}, \lambda\right)$, and then to normalize this by $G\left(e_{3}, e_{3}, \lambda\right)$. In this process is continued in the same way, it clearly produces an orthonormal set $\left\{G\left(e_{1}, e_{1}, \lambda\right), \ldots, G\left(e_{n}, e_{n}\right.\right.$, $\lambda), \ldots\}$ with the required property.

Annotation. We notice that the preceding orthogonalization process introduced by above as a special case contains well-known Gram-Schmidt orthogonalization process on Hilbert spaces.

The conjugate space $S^{*}$. We pointed out in the introduction of this problem that one of the fundamental properties of a complete general convex scalar space $S$ is the fact that there is a natural correspondence between the elements in $S$ and the functionals in $S^{*}$. In this sense we have the following result.

Theorem 29. Let $S$ be a complete general convex scalar space and let $f$ be an arbitrary functional in $S^{*}$. then there exists a unique element $G(y, y, \lambda)$ in $S$ such that

$$
f(G(x, x, \lambda))=(G(x, x, \lambda) \mid G(y, y, \lambda))
$$

for every $G(x, x, \lambda) \in S$. (The proof of this statement is founded on Theorem 26.)

The most important subsystem of the complex plane is the real line, which is characterized by the relation $z=\bar{z}$. By analogy, we can consider those operators $A$ on $S$ which equal their adjoints, that is, which satisfy the 
condition $A=A^{*}$. Such an operator is said to be self-adjoint. The selfadjoint operators on $S$ are evidently those which are related in the simplest possible way to their adjoints.

Proposition 10. An operator $T$ on a complete general convex scalar space $S$ is self-ajdoint if and only if $(T(G(x, x, \lambda)) \mid G(x, x, \lambda))$ is real for every $G(x, x, \lambda)$ in $S$.

This statement enables us to define a respectable and useful order relation on the set of all self-adjoint operators. If $A_{1}$ and $A_{2}$ are self-adjoint, we write $A_{1} \preccurlyeq A_{2}$ if $\left(A_{1}(G(x, x, \lambda)) \mid G(x, x, \lambda)\right) \leq\left(A_{2}(G(x, x, \lambda)) \mid G(x, x, \lambda)\right)$ for all $G(x, x, \lambda) \in S$.

A self-adjoint operator $A$ is said to be positive if $A \geq 0$, that is, if $(A(G(x, x, \lambda)) \mid G(x, x, \lambda)) \geq 0$ for all $G(x, x, \lambda) \in S$. It is clear that 0 and $I$ are positive, as are $T^{*} T$ and $T T^{*}$ for an arbitrary operator $T$.

An operator $N$ on a complete general convex scalar space $S$ is said to be normal if it commutes with its adjoint, that is, if $N N^{*}=N^{*} N$.

By definition, a self-adjoint operator $A$ is one which satisfies the identity of the form $A^{*}(G(x, x, \lambda))=A(G(x, x, \lambda))$. Many properties of self-adjoint operators do not depend on this, but only on the weaker identity of the form $\left\|A^{*}(G(x, x, \lambda))\right\|=\|A(G(x, x, \lambda))\|$.

Theorem 30. If $T$ is an operator on a complete general convex scalar space $S$, then the following conditions are all equivalent to one another: $T^{*} T=I$, $\|T(G(x, x, \lambda))\|=\|G(x, x, \lambda)\|$ for all $G(x, x, \lambda) \in S$, and

$$
(T(G(x, x, \lambda)) \mid T(G(y, y, \lambda)))=(G(x, x, \lambda) \mid G(y, y, \lambda))
$$

for all $G(x, x, \lambda), G(y, y, \lambda) \in S$. (The proof of this statement founded on the fact that $T=0$ if and only if $(T(G(x, x, \lambda)) \mid G(x, x, \lambda))=0$ for an operator $T$ on $S$.)

Projections. According to the definition given in the former part, a projection on a complete transversal upper normed space $S$ is an idempotent operator on $S$, that is, an operator $P$ with the property that $P^{2}=P$. Our first statement gives a convenient characterization of these projections.

Proposition 11. If $P$ is a projection on a complete general convex scalar space $S$ with range $M$ and null space $N$, then $M \perp N$ if and only if $P$ is self-adjoint; and in this $N=M^{\perp}$.

A projection on a complete general convex scalar space $S$ whose range and null space are orthogonal is sometimes called a perpendicular projection. In the light of this agreement and Pproposition 11, a projection on $S$ can be defined as an operator $P$ which satisfies the conditions $P^{2}=P$ and $P^{*}=P$. The operators 0 and $I$ are projections, and they are distinct if and only if $S \neq\{0\}$. 
If $T$ is an operator on a complete general convex scalar space $S$, then the simplest thing $T$ can do to an element $G(x, x, \lambda)$ is to transform it into a scalar multiple of itself:

$$
T(G(x, x, \lambda))=\alpha G(x, x, \lambda) .
$$

A nonzero element $G(x, x, \lambda)$ such that (14) is true for some scalar $\alpha$ is called an eigenelement of $T$, and a scalar $\alpha$ such that (14) holds for some nonzero $G(x, x, \lambda)$ is called an eigenvalue of $T$. Each eigenvalue has one or more eigenelements associated with it, and to each eigenelement there corresponds precisely one eigenvalue. If $S$ has no nonzero elements at all, then $T$ certianly has no eigenelements. In this case the whole theory collapses into triviality, so we assume throughout the present part that $S \neq\{0\}$.

Let $\alpha$ be an eigenvalue of $T$, and consider the set $M$ of all its corresponding eigenelements together with the element 0 (note that 0 is not an eigenelement). The set $M$ is thus the set of all elements $G(x, x, \lambda)$ which satisfy the equation

$$
(T-\alpha I) G(x, x, \lambda)=0
$$

and it is clearly a nonzero closed linear subspace of $S$. We call $M$ the eigen space of $T$ correpsonding to $\alpha$. It is evident that $M$ is invariant under $T$ and that the restriction of $T$ to $M$ is a very simple operator, namely, scalar multiplication by $\alpha$.

Our first goal is to prove that every operator on $S$ has an eigenvalue, and in pursuing this we make use of certain elementary portions of the theory of matrices.

Let $B=\left\{G\left(e_{1}, e_{1}, \lambda\right), \ldots, G\left(e_{n}, e_{n}, \lambda\right)\right\}$ be an ordered basis for $S$, so that each element in $S$ is uniquely expressible as a general convex linear combination of the $G\left(e_{i}, e_{i}, \lambda\right)^{\prime} s$. If $T$ is an operator on $S$, then for each $G\left(e_{j}, e_{j}, \lambda\right)$ we have

$$
T\left(G\left(e_{j}, e_{j}, \lambda\right)\right)=\sum_{i=1}^{n} \alpha_{i j} G\left(e_{i}, e_{i}, \lambda\right) .
$$

Then $n^{2}$ scalars $\alpha_{i j}$ which are determined in this way by $T$ form the matrix of $T$ relative to the ordered basis $B$. We symbolize this matrix by $[T]$ or if it seems desirable to indicate the ordered basis under consideration, by $[T]_{B}$. It is customary to write out a matrix as a square array:

$$
[T]:=\left[\begin{array}{cccc}
\alpha_{11} & \alpha_{12} & \ldots & \alpha_{1 n} \\
\alpha_{21} & \alpha_{22} & \ldots & \alpha_{2 n} \\
\ldots & \ldots & \ldots & \ldots \\
\alpha_{n 1} & \alpha_{n 2} & \ldots & \alpha_{n n}
\end{array}\right]
$$

In further work, we generally write (16) more concisely in the form $[T]=$ $\left[\alpha_{i j}\right]$. The term matrix has not been defined at all, but only "the matrix of an operator relative to an ordered basis". 
Theorem 31. If $B=\left\{G\left(e_{i}, e_{i}, \lambda\right)\right\}$ is a basis for $S$, then the mapping $T \mapsto[T]$, which assigns to each operator $T$ its matrix relative to $B$, is an isomorphism of the algebra $\mathcal{B}(S)$ onto the total matrix algebra $A_{n}$.

We now reverse our point of view for a moment (but only a moment) and consider the set $A_{n}$ of all $n \times n$ matrices as an algebric system in its own right, with addition, scalar multiplication and classical multiplication with matrixs. It can be verified directly from these definitions that $A_{n}$ is a complex algebra with identity (the identity matrix), called the total matrix algebra of degree $n$.

Theorem 32. Let $B$ be a basis for $S$, and $T$ an operator whose matrix relative to $B$ is $\left[\alpha_{i j}\right]$. Then $T$ is nonsingular if and only if $\left[\alpha_{i j}\right]$ is nonsingular, and in this case $\left[\alpha_{i j}\right]^{-1}=\left[T^{-1}\right]$.

There is one further issue which requires discussion. If $T$ is a fixed operator on $S$, then its matrix $[T]_{B}$ relative to $B$ obviously depends on the choice of $B$. If $B$ changes, how does $[T]_{B}$ change?! The ansfer to this question is best given in terms of the nonsingular operator $A$ defined by $A\left(G\left(e_{i}, e_{i}, \lambda\right)\right)=G\left(f_{i}, f_{i}, \lambda\right)$.

Theorem 33. If $T$ is an arbitrary operator on $S$, then the eigenvalues of $T$ constitute a nonempty finite subset of the complex plane. Furthemore, the number of points in this set does not exceed the dimension $n$ of the space $S$.

The set of eigenvalues of $T$ is called its spectrum, and is denoted by $\sigma(T)$. We observe that $\sigma(T)$ is a compact subspace of the complex plane.

The spectrum theorem. We now return to the central purpose of this part, namely, the statement and formulation of the spectral theorem.

Let $T$ be an arbitrary operator on $S$. We know by Theorem 33 that the distinct eigenvalues of $T$ are form a nonempty finite set of complex numbers. Let $\alpha_{1}, \alpha_{2}, \ldots, \alpha_{m}$ be these eigenvalues; let $M_{1}, M_{2}, \ldots, M_{m}$ be their corresponding eigenspaces; and let $P_{1}, P_{2}, \ldots, P_{m}$ be the projections on these eigenspaces. We consider the following three statements: (a) The $M_{i}$ 's are pairwise orthogonal and span $S$; (b) The $P_{i}$ 's are pairwise orthogonal, $I=\sum_{i=1}^{m} G\left(P_{i}, P_{i}, \lambda\right)$, and $T=\sum_{i=1}^{m} \alpha_{i} G\left(P_{i}, P_{i}, \lambda\right)$; and (c) $T$ is normal.

We take the spectral theorem (or general convex spectral theorem) to be the assertion that these statements ((a), (b), (c)) are all equivalent to one another. A brief proof of this result may be found in Tasković [22].

Linear optimization in $\mathbb{R}^{n}$. In this part all considerations are obtained by means of simple calculations with matrices. In this sense, we consider the following linear optimization problem for an arbitrary nonempty set $X$ 
in the form as

$$
\begin{gathered}
\inf _{u_{1}, \ldots, u_{n} \in X} \sum_{i=1}^{n} c_{i} G\left(u_{i}, u_{i}, \lambda\right)=a, \quad G\left(u_{i}, u_{i}, \lambda\right) \in \mathbb{R}_{+}^{n}, \\
r_{j}-\sum_{i=1}^{n} d_{j i} G\left(u_{i}, u_{i}, \lambda\right) \leq 0, \quad j=1, \ldots, m .
\end{gathered}
$$

Parallel to this, for existence theory, it turns out to be basic to study the following dual problem in the form as

$$
\begin{gathered}
\sup _{\alpha_{1}, \ldots, \alpha_{m} \in X} \sum_{j=1}^{m} r_{j} G\left(\alpha_{j}, \alpha_{j}, \lambda\right)=b, \quad G\left(\alpha_{j}, \alpha_{j}, \lambda\right) \in \mathbb{R}_{+}^{m}, \\
\sum_{j=1}^{m} d_{j i} G\left(\alpha_{j}, \alpha_{j}, \lambda\right)-c_{i} \leq 0, \quad i=1, \ldots, n .
\end{gathered}
$$

Here $G\left(u_{i}, u_{i}, \lambda\right) \in \mathbb{R}_{+}^{n}$ means that $G\left(u_{i}, u_{i}, \lambda\right) \geq 0$ for all $i=1, \ldots, n$. If we use $G\left(u_{i}, u_{i}, \lambda\right) \geq 0$ for $G\left(u_{i}, u_{i}, \lambda\right) \in \mathbb{R}_{+}^{n}$, then in matrix notation (17) and (18) read briefly in the following general form for $u=\left(u_{1}, \ldots, u_{n}\right)$, $r=\left(r_{1}, \ldots, r_{n}\right), c=\left(c_{1}, \ldots, c_{n}\right)$, and $\alpha=\left(\alpha_{1}, \ldots, \alpha_{n}\right)$ as follows:

$$
\inf _{u_{1}, \ldots, u_{n} \in X}(c \mid G(u, u, \lambda))=a, \quad G(u, u, \lambda) \geq 0, \quad r-D(G(u, u, \lambda)) \leq 0,
$$

$$
\sup _{1, \ldots, \alpha_{n} \in X}(r \mid G(\alpha, \alpha, \lambda))=b, \quad G(\alpha, \alpha, \lambda) \geq 0, \quad D^{*}(G(\alpha, \alpha, \lambda))-c \leq 0 .
$$

In after multiplying by -1 , we formulate (18), respectively (17), as a minimum problem (respectively, as a maximum problem), then, because $D^{* *}=D$, one immediately recognizes that (19) is the problem dual to (20).

An optimization problem is said to be consistent if and only if the feasible region is not empty. This is a trivial requirement for the existence of a solution. The question arises whether the following is valid: Consistency implies Existence.

The simple example in $\mathbb{R},-u=\min$ ! for $u \geq 0$ shows that a consistent problem need not have a solution. However, the following our statement of general convex linear oprimization shows that the existence of solutions for both problems follows from the consistency of the original problem and of the dual problem.

Theorem 34. The following three statements are equivalent: (a) The original problem (17) has a solution. (b) The dual problem (18) has a solution. (c) Both problems are consistent. If any one of these conditions holds, then, moreover, $a=b$.

In connection with this, all considerations are obtained by means of simple calculations with matrices. In the following, let $A: \mathbb{R}^{n} \rightarrow \mathbb{R}^{m}$ be a general 
convex linear operator, i.e., $A$ can be is equal to a real matrix $\left[\alpha_{i j}\right]$, and $X$ be a nonempty set. The adjoint matrix corresponds to $A^{*}$. Furthemore, we recall that for $u, v \in X^{n}$ and $G(u, v, \lambda) \in \mathbb{R}^{n}$, we have

$$
(G(u, u, \lambda) \mid G(v, v, \lambda))=\sum_{i=1}^{n} G\left(u_{i}, u_{i}, \lambda\right) G\left(v_{i}, v_{i}, \lambda\right),
$$

where $u=\left(u_{1}, \ldots, u_{n}\right), v=\left(v_{1}, \ldots, v_{n}\right), G\left(u_{i}, u_{i}, \lambda\right) \in \mathbb{R}_{+}^{n}$, and $G\left(v_{i}, v_{i}, \lambda\right) \in$ $\mathbb{R}_{+}^{n}$. In this context, the problem of the following form as

$$
A(G(u, u, \lambda))=a, \quad \text { for } \quad G(u, u, \lambda) \geq 0,
$$

has a solution $G(u, u, \lambda) \in \mathbb{R}^{n}$ if and only if the following inequality holds in the form as

$$
(G(v, v, \lambda) \mid a) \geq 0 \quad \text { for all } G(v, v, \lambda) \text { with } A^{*}(G(v, v, \lambda)) \geq 0 .
$$

We notice that this statement is a generalization of the well known criterions for the solution of a system of linear equations. (In this sense, (21) has a solution $G(u, u, \lambda)$ for some $u \in X^{n}$ if and only if $(G(v, v, \lambda) \mid a)=0$ for all $G(v, v, \lambda)$ with $\left.A^{*}(G(v, v, \lambda))=0\right)$. Indeed, (21) implies (22) because $\left(A^{*}(G(v, v, \lambda)) \mid G(u, u, \lambda)\right)=$ $(G(v, v, \lambda) \mid A(G(u, u, \lambda)))=(G(v, v, \lambda) \mid a)$. Also, we have and that (22) implies (21). For this see: Tasković [22].

An alternative of the preceding result. In this sense we have the following result: Either (21) has a solution $G(u, u, \lambda) \in \mathbb{R}^{n}$ or the inequality of the form as

$$
A^{*}(G(v, v, \lambda)) \geq 0, \quad \text { for } \quad(G(v, v, \lambda) \mid a)<0,
$$

has a solution $G(v, v, \lambda) \in \mathbb{R}^{n}$ for some $v \in X^{n}$. (Both the preceding results, in the special case, contains result of: F a r k a s [1902]. Also see: G o r d a n [1873], Dubovickij - Miljutin [1965], Craven [1978], Girsanov [1972], Kuhn - Tucker [1951], and Minkowski [1911]).

An example. Let $a_{i j}$ for $i=1, \ldots, m$ and $j=1, \ldots, n$ be real numbers, and let $b_{1}, \ldots, b_{m}$ be also real numbers. Determined elements $G\left(x_{i}, x_{i}, \lambda\right) \in \mathbb{R}$ $(i=1, \ldots, n)$ for $x_{1}, \ldots, x_{n} \in X$ (= a nonemty set) such that

$$
\sum_{j=1}^{n} a_{i j} G\left(x_{j}, x_{j}, \lambda\right) \leq b_{i} \quad(i=1, \ldots, m) .
$$

In this context, we have that with $(23)$ is given a system of $m$ inequalities with $G\left(x_{1}, x_{1}, \lambda\right), \ldots, G\left(x_{n}, x_{n}, \lambda\right)$ as unknowns for $x_{1}, \ldots, x_{n} \in X$. If we set that is

$$
G\left(x_{n+i}, x_{n+i}, \lambda\right)=b_{i}-\sum_{j=1}^{n} a_{i j} G\left(x_{j}, x_{j}, \lambda\right) \quad \text { for } \quad i=1, \ldots, m ;
$$

then system of inequalities of the form (23) teasing in the system of $m$ equalities of the following form as

$$
\sum_{j=1}^{n} a_{i j} G\left(x_{j}, x_{j}, \lambda\right)+G\left(x_{n+i}, x_{n+i}, \lambda\right)=b_{i} \quad \text { for } \quad i=1, \ldots, m
$$

with $n+m$ unknowns. Every solution of (23) under (24) to bring on solution of (25). Vice versa, if $G\left(c_{1}, \ldots, c_{n}\right)$ for $c_{1}, \ldots, c_{n} \in X$ is a solution of (25) and if 
$G\left(c_{n+1}, c_{n+1}, \lambda\right), \ldots, G\left(c_{n+m}, c_{n+m}, \lambda\right) \geq 0$, then $G\left(c_{1}, c_{1}, \lambda\right), \ldots, G\left(c_{n}, c_{n}, \lambda\right)$ is a solution of (23) for $c_{1}, \ldots, c_{n} \in X$.

In connection with the preceding facts, for a linear subspace $L$ of a general convex linear space $Y$ we have $L^{+}:=\left\{f \in Y^{*}: f(G(x, x, \lambda))=0\right.$ on $\left.L\right\}$. Also, for $K:=\{(x, y) \in X \times Y: B(G(x, x, \lambda))=G(y, y, \lambda)\}$ we have the following dual cone to $K$ in the form as

$$
\left.K^{+}=\left\{\left(x^{*}, y^{*}\right) \in X^{*} \times Y^{*}: G\left(x^{*}, x^{*}, \lambda\right)=-B^{*}\left(y^{*}, y^{*}, \lambda\right)\right)\right\} .
$$

Proposition 12. Let $X$ be a nonempty set and $L$ be a linear subspace of a general convex linear space $Y$ and $B: Y \rightarrow Y$. Then for the set of the following form as

$$
K:=\left\{x \in X: B(G(x, x, \lambda)) \in K_{Y}\right\} \equiv B^{-1}\left(K_{Y}\right)
$$

we have $K^{+}=B^{*}\left(K_{Y}^{+}\right)$, when $K_{Y}$ is general convex and either one of the following two conditions is satisfied: (a) There exists an $x_{0} \in X$ such that $B\left(G\left(x_{0}, x_{0}, \lambda\right)\right) \in \operatorname{intK}_{Y}$; (b) $X=\mathbb{R}^{n}, Y=\mathbb{R}^{m}, K_{Y}=\mathbb{R}_{+}^{m}$.

Annotations. We notice that, in the case (b), we have $K^{+}=\left\{B^{*}(G(y, y, \lambda))\right.$ : $\left.G(y, y, \lambda) \in \mathbb{R}_{+}^{m}\right\}$ because $K_{Y}^{+}=K_{Y}$. This relation can be also expressed as follows: The system

$$
B^{*}(G(y, y, \lambda))=b \quad \text { for } \quad G(y, y, \lambda) \geq 0
$$

has a solution $G(y, y, \lambda)$ if and only if $b \in K^{+}$, i.e., if and only if the following inequality holds in the form as

$$
(b \mid G(x, x, \lambda)) \geq 0 \quad \text { with } \quad B(G(x, x, \lambda)) \geq 0 \quad \text { for all } x \in X .
$$

Otherwise, in this form of Proposition 12, directly as a special case we obtain the Farkas lemma as and a result well-known as Minkowski lemma. Also, in the special case we have the following result: If $\mathrm{K}$ consists of all $G(x, x, \lambda) \in \mathbb{R}^{n}$ for $x \in \mathbb{R}$ with

$$
\left(a_{i} \mid G(x, x, \lambda)\right) \geq 0 \quad \text { for } \quad i=1, \ldots, k
$$

and

$$
\left(a_{i} \mid G(x, x, \lambda)\right)=0 \quad \text { for } \quad i=k+1, \ldots, m
$$

for fixed $a_{i} \in \mathbb{R}^{n}$, then $K^{+}$consists of all $f \in \mathbb{R}^{n}$, where with $\lambda_{i} \in \mathbb{R}(i=1, \ldots, m)$ $f$ satisfy the following equality in the form as

$$
f=\sum_{i=1}^{m} a_{i} G\left(\lambda_{i}, \lambda_{i}, \lambda\right) \quad \text { for } \quad \lambda_{1}, \ldots, \lambda_{k} \geq 0 .
$$

Iterative method for general convex linear equations. Our subject will be the general convex linear operator equation

$$
G(x, x, \lambda)=A(G(x, x, \lambda))+b \quad \text { for } \quad x \in X
$$

and the corresponding iterative method in the following form as

$$
G\left(x_{n}, x_{n}, \lambda\right)=A\left(G\left(x_{n-1}, x_{n-1}, \lambda\right)\right)+b, \quad x_{0} \in X \quad \text { and } n \in \mathbb{N},
$$


where given are the continuous general convex linear operator $A: Y \rightarrow Y$ on a complete general convex linear space $Y$, and the element $b \in Y$. The conclusions below regarding

$$
G(x, x, \lambda)=A(G(x, x, \lambda))+b \quad \text { for } x \in X,
$$

where $X$ is an arbitrary nonempty set and (Mi) are of great importance for linear problems. In this sense, equation (26) has a stable iterative method if (26) has exactly one solution $G(x, x, \lambda) \in Y$ for each $b \in Y$ and if the iterative sequence $\left\{G\left(x_{n}, x_{n}, \lambda\right)\right\}_{n \in \mathbb{N}}$ converges to $G(x, x, \lambda)$ from an arbitrary initial element $x_{0} \in X$.

Theorem 35. (General convex linear equations). Supposes that $A: Y \rightarrow Y$ is a continuous general convex linear operator on a complete general convex vector space $Y$ and let $\|A\|<1$. Then (26) has a stable iterative method. The inverse $(I-A)^{-1}$ exists as a continuous general convex linear operator on $Y$ and

$$
(I-A)^{-1}=\sum_{k=0}^{\infty} A^{k}
$$

by that this generalized geometric series (i.e., Neumann's series) converges in the operator norm. The unique solution of (26) is $G(x, x, \lambda)=(I-A)^{-1} b$ for some $x \in X$. Also, if the spectral radius $r(A)<1$, then (26) has a stable iterative method.

This statement has a multitude of applications to linear systems of equations and to linear integral equations. The iterative solution of linear systems of equations is of central importance for a practical treatment of difference methods for ordinary and partial differential equations. See: Tasković [22].

Nonlinear optimization. In this part we will explain the connection between the general duality theory and Kuhn - Tucker theory with general convexity. For this purpose we consider our original problem to be

$$
\inf _{u \in A} F(G(u, u, \lambda))=\alpha \quad \text { for } \quad G(u, u, \lambda) \in A,
$$

and for $F_{j}(G(u, u, \lambda)) \leq 0$ for $j=1, \ldots, n$ by that the corresponding dual problem of this problem which has the following form as

$$
\sup _{p \in \mathbb{R}_{+}^{n}}\left(\inf _{u \in A} L(u, p)\right)=\beta,
$$

where

$$
L(u, p):=F(G(u, u, \lambda))+\sum_{j=1}^{n} p_{j} F_{j}(G(u, u, \lambda)) .
$$

Proposition 13. Suppose the following two conditions hold: (a) $A$ is a closed general convex nonempty set in the real reflexive complete normed general convex linear space $X$, (b) the functionals $F, F_{1}, \ldots, F_{n}: A \subset$ 
$X \rightarrow \mathbb{R}$ are transversal general convex and lower semicontinuous. Then (28) always has a solution $p$ and $\alpha=\beta$; also, the following two assertion are equivalent: $u \in A$ is a solution of the original problem (27) and $L$ has a saddle point $(u, p)$ with respect to $A \times \mathbb{R}_{+}^{n}$.

Annotation. We notice that in the case if $L$ has a saddle point $(u, p)$, then $p \in \mathbb{R}_{+}^{n}$ is a solution of the dual problem (28) and the following fact holds in the form as

$$
p_{j} F_{j}(G(u, u, \lambda))=0 \quad \text { for } \quad j=1, \ldots, n .
$$

The proof ot this statement may be found in: Tas k ović [22]. Also, a variant of the proof of this statement may be found in $\mathrm{Z}$ e i d l e r [41].

Transversal Convex Functions. In this section we continue the study of the general convex functions which are introduced in our former paper [T a s k o v i ć: Math. Japonica, 37 (1992), 367-372]. In this sense, we introduced and considered a new concept of convexity via a concept of transversal functions on a transversal general convex subset $D$ in a general convex linear space $E$.

In further, a function $f: D \rightarrow \mathbb{R}(D \subset E$ is a transversal general convex set) is said to be transversal general convex function if there is a function $g: f(D)^{2} \rightarrow \mathbb{R}$ such that

(Max)

$$
\begin{gathered}
f(G(x, y, \lambda)) \leq \\
\leq \max \{f(G(x, x, \lambda)), f(G(y, y, \lambda)), g(f(G(x, x, \lambda)), f(G(y, y, \lambda)))\}
\end{gathered}
$$

for all elements $x, y \in X$ (where $X$ is an arbitrary nonempty set) and for an arbitrary $\lambda \in[0,1]$.

In this general case, if $G(x, y, \lambda)=\lambda x+(1-\lambda) y$ for arbitrary $\lambda \in[0,1]$ and for all $x, y \in D$, then we obtain a well known subclasse denoted by general convex functions by Tasković [1992] in the form as

$$
f(\lambda x+(1-\lambda) y) \leq \max \{f(x), f(y), g(f(x), f(y))\} .
$$

A transversal general convex funcation $f: D \rightarrow \mathbb{R}(D \subset E$ is a transversal general convex set) is said to be strictly transversal general convex if for every pair of elements $x, y \in X(x \neq y)$ and for an arbitrary $\lambda \in[0,1]$ strict inequality holds in (Max).

On the other hand, $f: D \rightarrow \mathbb{R}(D \subset E$ is a transversal general convex set) is called proper transversal $\mathbf{J}$-general convex function if there is a function $g: f(D)^{2} \rightarrow \mathbb{R}$ such that

$$
\begin{gathered}
f\left(\frac{G(x, y, \lambda)}{2}\right) \leq \\
\leq \max \{f(G(x, x, \lambda)), f(G(y, y, \lambda)), g(f(G(x, x, \lambda)), f(G(y, y, \lambda)))\}
\end{gathered}
$$

for all elements $x, y \in X$ (where $X$ is an arbitrary nonempty set) and for an arbitrary $\lambda \in[0,1]$. A function $f: D \rightarrow \mathbb{R}(D \subset E$ is a transversal general convex set) is called transversal $\mathbf{J}$-general convex function if $G(x, y, \lambda)=$ $G(x, x, \lambda)+G(y, y, \lambda)$ in the inequality (29). 
If $G(x, y, \lambda)=(x+y) / 2$ in the inequality (Max), then we obtain a well known subclasse denoted by general J-convex functions by Task ović [1992] in the form as

$$
f\left(\frac{x+y}{2}\right) \leq \max \{f(x), f(y), g(f(x), f(y))\}
$$

for all $x, y \in D$. Otherwise, a transversal J-general convex function $f: D \rightarrow \mathbb{R}$ ( $D \subset E$ is a transversal general convex set) is called strictly transversal Jgeneral convex if for every pair of elements $x, y \in X(x \neq y)$ and for an arbitrary $\lambda \in[0,1]$ strict inequality holds in (29).

In connection with the preceding, a function $f: D \rightarrow \mathbb{R}(D \subset E$ is a transversal general convex set) is said to be transversal reversed general convex function if there is a function $d: f(D)^{2} \rightarrow \mathbb{R}$ such that

(Min)

$$
\begin{gathered}
f(G(x, y, \lambda)) \geq \\
\geq \min \{f(G(x, x, \lambda)), f(G(y, y, \lambda)), d(f(G(x, x, \lambda)), f(G(y, y, \lambda)))\}
\end{gathered}
$$

for all elements $x, y \in X$ (where $X$ is an arbitrary nonempty set) and for an arbitrary $\lambda \in[0,1]$.

A transversal reversed general convex function $f: D \rightarrow \mathbb{R}(D \subset E$ is a transversal general convex set) is said to be strictly transversal reversed general convex if for every pair of elements $x, y \in X(x \neq y)$ and for an arbitrary $\lambda \in[0,1]$ strict inequality holds in (Min).

If $G(x, y, \lambda)=\lambda x+(1-\lambda) y$ for arbitrary $\lambda \in[0,1]$ and for all $x, y \in D$, then we obtain a well known subclasse denoted by general concave functions by Tasković [1992] in the form

$$
f(\lambda x+(1-\lambda) y) \geq \min \{f(x), f(y), d(f(x), f(y))\} .
$$

Also, $f: D \rightarrow \mathbb{R}$ ( $D \subset E$ is a transversal general convex set) is called proper transversal reversed J-general convex function if there is a function $d$ : $f(D)^{2} \rightarrow \mathbb{R}$ such that

$$
\begin{gathered}
f\left(\frac{G(x, y, \lambda)}{2}\right) \geq \\
\geq \min \{f(G(x, x, \lambda)), f(G(y, y, \lambda)), d(f(G(x, x, \lambda)), f(G(y, y, \lambda)))\}
\end{gathered}
$$

for all elements $x, y \in X$ (where $X$ is an arbitrary nonempty set) and for an arbitrary $\lambda \in[0,1]$. A function $f: D \rightarrow \mathbb{R}(D \subset E$ is a transversal general convex set) is called transversal reversed $\mathbf{J}$-general convex if $G(x, y, \lambda)=$ $G(x, x, \lambda)+G(y, y, \lambda)$ in the inequality (30).

If $G(x, y, \lambda)=(x+y) / 2$ in the inequality (Min), then we obtain a well known subclasse denoted by general J-concave functions by Tasković [1992] in the form as

$$
f\left(\frac{x+y}{2}\right) \geq \min \{f(x), f(y), d(f(x), f(y))\}
$$

for all points $x, y \in D$. Otherwise, a transversal reversed J-general convex function $f: D \rightarrow \mathbb{R}(D \subset E$ is a transversal general convex set $)$ is called strictly transversal reversed $\mathbf{J}$-general convex if for every pair of elements $x, y \in X(x \neq y)$ and for an arbitrary $\lambda \in[0,1]$ strictly inequality holds in (30). 
Some subclasses of transversal convex functions. In this sense, a function $f: D \rightarrow \mathbb{R}(D \subset E$ is a transversal general convex set) is called transversal convex iff the following inequality holds in the form as

$$
f(G(x, y, \lambda)) \leq \alpha f(G(x, x, \lambda))+(1-\alpha) f(G(y, y, \lambda))
$$

for all elements $x, y \in X$ (where $X$ is an arbitrary nonempty set) and for all $\alpha \in[0,1]$ and $\lambda \in[0,1]$.

A transversal convex function $f: D \rightarrow \mathbb{R}$ is said to be strictly transversal convex if strict inequality holds in $(31)$ for all $x, y \in X(x \neq y)$, for all $\alpha \in[0,1]$ and $\lambda \in[0,1]$.

In this general case, if $G(x, y, \lambda)=\lambda x+(1-\lambda) y$ for every $\lambda=\alpha \in[0,1]$ in (31), then we obtain well known class of convex functions.

On the other hand, a function $f: D \rightarrow \mathbb{R}(D \subset E$ is a transversal general convex set) is called proper transversal $\mathbf{J}$-convex if the following inequality holds in the form as

$$
f\left(\frac{G(x, y, \lambda)}{2}\right) \leq \frac{f(G(x, x, \lambda))+f(G(y, y, \lambda))}{2}
$$

for all elements $x, y \in X$ (where $X$ is and arbitrary nonempty set) and for an arbitrary $\lambda \in[0,1]$.

A function $f: D \rightarrow \mathbb{R}(D \subset E$ is a transversal general convex set) is called transversal J-convex function if $G(x, y, \lambda)=G(x, x, \lambda)+G(y, y, \lambda)$ in the inequality (32).

If $G(x, y, \lambda)=(x+y) / 2$ in the inequality $(31)$, then we obtain a well known subclasse denoted by J-convex functions by J e n s e n [1905].

Otherwise, a proper transversal J-convex function $f: D \rightarrow \mathbb{R}(D \subset E$ is a transversal general convex set) is called strictly proper transversal J-convex if for every pair of elements $x, y \in X(x \neq y)$ and for an arbitrary $\lambda \in[0,1]$ strict inequality holds in (32).

Inequalities of transversal J-general convexity. We begin with the following essential statement in the form as.

Theorem 36. Let $D \subset \mathbb{R}^{n}$ be a transversal general convex and open set. If $f: D \rightarrow \mathbb{R}$ is a transversal J-general convex function, then there is a function $g: f(D)^{2} \rightarrow \mathbb{R}$ such that

$$
f\left(\frac{G\left(x_{1}, x_{1}, \lambda\right)+\cdots+G\left(x_{n}, x_{n}, \lambda\right)}{n}\right) \leq
$$

$\leq \max \left\{f\left(G\left(x_{1}, x_{1}, \lambda\right)\right), \ldots, f\left(G\left(x_{n}, x_{n}, \lambda\right)\right), g\left(f\left(G\left(x_{1}, x_{1}, \lambda\right)\right), \ldots, f\left(G\left(x_{n}, x_{n}, \lambda\right)\right)\right)\right\}$

for every $n \in \mathbb{N}$, for all elements $x_{1}, \ldots, x_{n} \in X$ (where $X$ is an arbitrary nonempty set) and for an arbitrary $\lambda \in[0,1]$. Equality holds in $(D N)$ if and only if $x:=x_{1}=$ $\cdots=x_{n}$ and $f(G(x, x, \lambda))=g(f(G(x, x, \lambda)), \ldots, f(G(x, x, \lambda)))$.

Induction shows that this statement holds. As an immediate consequence of Theorem 36 we obtain the following statement.

Theorem 37. Let $D \subset \mathbb{R}^{n}$ be a transversal general convex and open set. If $f: D \rightarrow \mathbb{R}$ is a transversal J-general convex function, then there is a function 
$g: f(D)^{2} \rightarrow \mathbb{R}$ such that

$$
\begin{gathered}
f(\alpha G(x, x, \lambda)+(1-\alpha) G(y, y, \lambda)) \leq \\
\leq \max \{f(G(x, x, \lambda)), f(G(y, y, \lambda)), g(f(G(x, x, \lambda)), f(G(y, y, \lambda)))\}
\end{gathered}
$$

for all elements $x, y \in X$ (where $X$ is an arbitrary nonempty set), for every rational number $\alpha \in \mathbb{Q} \cap[0,1]$, and for every $\lambda \in[0,1]$.

We notice, if $f: D \rightarrow \mathbb{R}$ is a transversal J-general convex and continuous function, then (Q) holds for all real numbers $\alpha \in[0,1]$.

Next, we give an interesting inequality for transversal J-general convex functions which is, in some sense, the best of its kind.

Theorem 38. Let $D \subset \mathbb{R}^{n}$ be a transversal general convex set and let $F \subset \mathbb{R}$ be a field. If a function $f: D \rightarrow \mathbb{R} \cup\{-\infty\}$ satisfies ( $Q$ ) for every $\alpha \in F \cap[0,1]$, then

$$
f\left(\sum_{j=1}^{n} \lambda_{j} G\left(x_{j}, x_{j}, \lambda\right)\right) \leq
$$

$\leq \max \left\{f\left(G\left(x_{1}, x_{1}, \lambda\right)\right), \ldots, f\left(G\left(x_{n}, x_{n}, \lambda\right)\right), g\left(f\left(G\left(x_{1}, x_{1}, \lambda\right)\right), \ldots, f\left(G\left(x_{n}, x_{n}, \lambda\right)\right)\right)\right\}$

for some function $g: f(D)^{2} \rightarrow \mathbb{R}$, for every $n \in \mathbb{N}$, for arbitrary $\lambda_{1}, \ldots, \lambda_{n} \in$ $F \cap[0,1]$ such that $\lambda_{1}+\cdots+\lambda_{n}=1$ and for all elements $x_{1}, \ldots, x_{n} \in X$ (where $X$ is an arbitrary nonempty set).

Thus inequality (D') with arbitrary $x_{1}, \ldots, x_{n} \in X$ is valid for every transversal J-general convex function $f: D \rightarrow \mathbb{R}$ with arbitrary $\lambda_{1}, \ldots, \lambda_{n} \in \mathbb{Q} \cap[0,1]$ adding up to 1 , and for every continuous transversal J-general convex function $f: D \rightarrow \mathbb{R}$ with arbitrary $\lambda_{1}, \ldots, \lambda_{n} \in[0,1]$ adding up to 1 .

Theorem 39. (Complementary inequality of (D')). Let $D \subset \mathbb{R}^{n}$ be a transversal general convex set which including zero and let $F \subset \mathbb{R}$ be a field. If $f: D \rightarrow$ $\mathbb{R} \cup\{-\infty\}$ for some $2 \leq \rho \leq n$ and for all $\lambda_{1}, \ldots, \lambda_{\rho} \in F \cap[0,1]$ such that $\lambda_{1}+\cdots+\lambda_{\rho}=1$ satisfy the following condition of the form as

$$
f\left(\sum_{j=1}^{\rho} \lambda_{j} G\left(x_{j}, x_{j}, \lambda\right)\right) \leq
$$

$\leq \max \left\{f\left(G\left(x_{1}, x_{1}, \lambda\right)\right), \ldots, f\left(G\left(x_{\rho}, x_{\rho}, \lambda\right)\right), g\left(f\left(G\left(x_{1}, x_{1}, \lambda\right)\right), \ldots, f\left(G\left(x_{\rho}, x_{\rho}, \lambda\right)\right)\right)\right\}$

for some function $g: f(D)^{\rho} \rightarrow \mathbb{R}$ and for all elements $x_{1}, \ldots, x_{n} \in X$ (where $X$ is an arbitrary nonempty set), then $f$ is a transversal J-general convex function.

In the next we give two characteristic examples for inequalities of subclasses of the class of transversal J-general convex functions.

Corollary 1. Let $D \subset \mathbb{R}^{n}$ be a transversal general convex and open set. If $f$ : $D \rightarrow \mathbb{R}$ is a transversal J-convex function, then the following inequality holds in the form as

$$
f\left(\frac{G\left(x_{1}, x_{1}, \lambda\right)+\cdots+G\left(x_{n}, x_{n}, \lambda\right)}{n}\right) \leq \frac{f\left(G\left(x_{1}, x_{1}, \lambda\right)\right)+\cdots+f\left(G\left(x_{n}, x_{n}, \lambda\right)\right)}{n}
$$


for every $n \in \mathbb{N}$, for all elements $x_{1}, \ldots, x_{n} \in X$ (where $X$ is an arbitrary nonempty set) and for an arbitrary $\lambda \in[0,1]$. Equality holds in (33) if and only if $x_{1}=\cdots=$ $x_{n}:=x$.

We can induction shows that this statement holds independent of Theorem 36 . On the other hand, from Theorem 37, Theorem 38 and Corollary 1 we have the following inequality for transversal J-convex functions in the form as.

Corollary 2. Let $D \subset \mathbb{R}^{n}$ be a transversal general convex set and let $F \subset \mathbb{R}$ be a field. If $f: D \rightarrow \mathbb{R} \cup\{-\infty\}$ satisfy the inequality $(Q)$ in the corresponding form for every $\alpha \in F \cap[0,1]$, then the following inequality holds in the form

$$
f\left(\sum_{j=1}^{n} \lambda_{j} G\left(x_{j}, x_{j}, \lambda\right)\right) \leq \sum_{j=1}^{n} \lambda_{j} f\left(G\left(x_{j}, x_{j}, \lambda\right)\right)
$$

for every $n \in \mathbb{N}$, for arbitrary numbers $\lambda_{1}, \ldots, \lambda_{n} \in F \cap[0,1]$ with the property $\lambda_{1}+\cdots+\lambda_{n}=1$ and for all elements $x_{1}, \ldots, x_{n} \in X$ (where $X$ is an arbitrary nonempty set).

In the context of the preceding statement immediate we obtain the following consequence of Theorem 38 and Corollary 2 for transversal convex functions.

Corollary 3. Let $D \subset \mathbb{R}^{n}$ be a transversal general convex and open set. If $f$ : $D \rightarrow \mathbb{R}$ is a transversal convex function, then the following inequality holds in the form as

$$
f\left(\frac{\sum_{j=1}^{n} p_{j} G\left(x_{j}, x_{j}, \lambda\right)}{\sum_{j=1}^{n} p_{j}}\right) \leq \frac{\sum_{j=1}^{n} p_{j} f\left(G\left(x_{j}, x_{j}, \lambda\right)\right)}{\sum_{j=1}^{n} p_{j}}
$$

for every $n \in \mathbb{N}$, for all elements $x_{1}, \ldots, x_{n} \in X$ (where $X$ is a nonempty set) and for arbitrary nonegative numbers $p_{1}, \ldots, p_{n} \in \mathbb{R}$ with the property $p_{1}+\cdots+p_{n}>0$.

As an immediate application of the transversal general convex functions we obtain the following form of Hahn-Banach type theorem.

Theorem 40. (General Hahn-Banach theorem). Let $X$ be a real general convex linear space, let $D \subset X$ be a subspace, and let $f: X \rightarrow \mathbb{R}$ be a transversal general convex function such that

$$
h(G(x, x, \lambda)) \leq f(G(x, x, \lambda)) \text { for every } x \in D,
$$

where $h: D \rightarrow \mathbb{R}$ is a general convex linear functional and $\lambda \in[0,1]$. Then there is a general convex linear functional $H: X \rightarrow \mathbb{R}$ such that

$$
H(G(x, x, \lambda)) \leq f(G(x, x, \lambda)) \quad \text { for every } x \in X,
$$

where $\lambda \in[0,1]$, and $H \mid D=h$, i.e., there is a general convex linear extension of the general convex linear functional $h$ on $X$.

General convex relativistic physics. Consider two systems $\sigma$ and $\sigma^{\prime}$ with corresponding space elements of general convex structures $G(x, x, \lambda)$, $G(y, y, \lambda), G(z, z, \lambda), G\left(x^{\prime}, x^{\prime}, \lambda\right), G\left(y^{\prime}, y^{\prime}, \lambda\right)$ and $G\left(z^{\prime}, z^{\prime}, \lambda\right)$. Assume also 


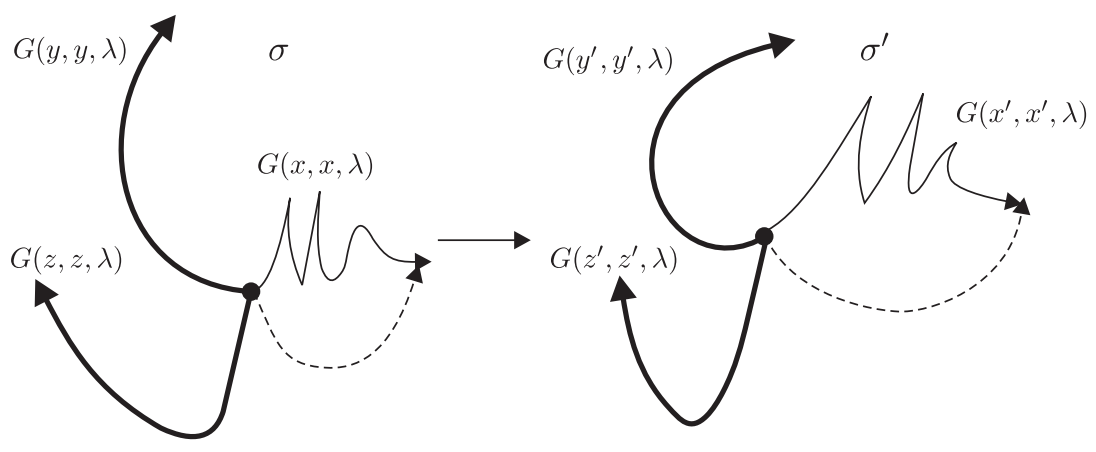

FiguRE 6.

that $\sigma$ and $\sigma^{\prime}$ are two transversal convex inertial systems of the form as on Fig. 6 with corresponding system times $G(t, t, \lambda)$ and $G\left(t^{\prime}, t^{\prime}, \lambda\right)$.

In this sense, a system $\sigma$ is a transversal general convex system precisely if there exists a system time $G(t, t, \lambda)$ for it such that each mass point, which is for enough away from other masses and shielded against fields, e. g., light pressure, remains at rest or moves rectilinearly with constant velocity.

At the beginning of this section I formulated the following three postulates in the corresponding form as:

(A) All transversal general convex inertial systems are physically equivalent, i.e., physical procesess are the same in all transversal general convex inertial systems when initial boundary conditions are the same.

(B) (Constant velocity of light). In every transversal general convex inertial system, light travels with the same constant velocity $G(c, c, \lambda)$ in every direction, where $c$ is the speed of light.

(C) (Principle of translation). There exists a transversal general convex inertial system. If $\sigma$ is a transversal general convex inertial system, then also each transversal general convex system $\sigma^{\prime}$, which is obtained from $\sigma$ by constant translatory motion, is a transversal general convex inertial system.

Recall that we mean by a translatory motion that $\sigma^{\prime}$ is not rotated compared with $\sigma$. By a constant translatory motion of $\sigma^{\prime}$ we mean a constant motion of $\sigma^{\prime}$ with tespect to $\sigma$ with constant velocity element $v$ as on Fig. 7 .

With the choice of the corresponding system $\sigma$ shown in Fig. 6, the corresponding structure element $G(y, y, \lambda)=0$ coincides with the element $G\left(y^{\prime}, y^{\prime}, \lambda\right)=0$ and the element $G(z, z, \lambda)=0$ with the element $G\left(z^{\prime}, z^{\prime}, \lambda\right)=$ 0 in the system $\sigma^{\prime}$.

Therefore, $G(y, y, \lambda)$ and $G\left(y^{\prime}, y^{\prime}, \lambda\right)$ can be related only by expressions (from the former general convex linear transformations) of the kind

$$
G(y, y, \lambda)=\alpha G\left(y^{\prime}, y^{\prime}, \lambda\right),
$$




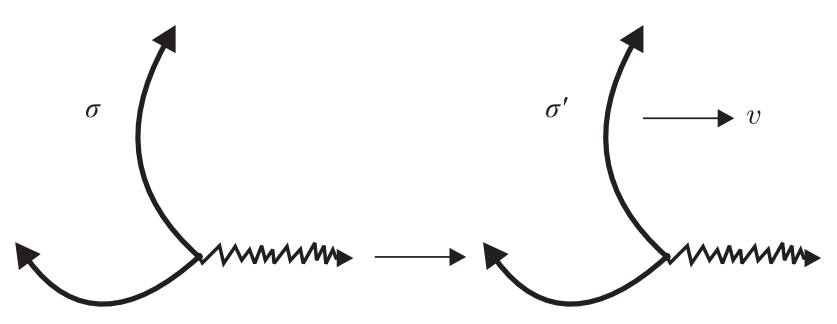

FigURE 7.

where $\alpha$ is a constant. Owing to the frames $\sigma$ and $\sigma^{\prime}$ having equal rights, the reverse relation must hold, i.e.,

$$
G\left(y^{\prime}, y^{\prime}, \lambda\right)=\alpha G(y, y, \lambda)
$$

with the same value of the constant $\alpha$ as in the first case. Multiplication of these two equations (34) and (35) yields $\alpha^{2}=1$, whence $\alpha=$ \pm 1 . The plus sign corresponds to the $G(y, y, \lambda)$ and $G\left(y^{\prime}, y^{\prime}, \lambda\right)$ having the same "directions", and the minus sign to their having opposite "directions". We get $G(y, y, \lambda)=G\left(y^{\prime}, y^{\prime}, \lambda\right)$, and similar reasoning yields $G(z, z, \lambda)=G\left(z^{\prime}, z^{\prime}, \lambda\right)$.

In further, let us turn to finding the transformations for $G(x, x, \lambda)$ and $G(t, t, \lambda)$. It can be seen from the preceding two equations that the values of $G(y, y, \lambda)$ and $G(z, z, \lambda)$ do not depend on $G\left(x^{\prime}, x^{\prime}, \lambda\right)$ and $G\left(t^{\prime}, t^{\prime}, \lambda\right)$. Hence, the values of $G\left(x^{\prime}, x^{\prime}, \lambda\right)$ and $G\left(t^{\prime}, t^{\prime}, \lambda\right)$ cannot depend on $G(y, y, \lambda)$ and $G(z, z, \lambda)$, correspondingly, the values of $G(x, x, \lambda)$ and $G(t, t, \lambda)$ cannot depend on $G\left(y^{\prime}, y^{\prime}, \lambda\right)$ and $G\left(z^{\prime}, z^{\prime}, \lambda\right)$. Thus, $G(x, x, \lambda)$ and $G(t, t, \lambda)$ can be general convex linear functions of only $G\left(x^{\prime}, x^{\prime}, \lambda\right)$ and $G\left(t^{\prime}, t^{\prime}, \lambda\right)$.

The origin of elements 0 of the system $\sigma$ has the value $G(x, x, \lambda)=0$ in the system $\sigma$ and $G\left(x^{\prime}, x^{\prime}, \lambda\right)=-v G\left(t^{\prime}, t^{\prime}, \lambda\right)$ in the system $\sigma^{\prime}$. Consequently, the expression $G\left(x^{\prime}, x^{\prime}, \lambda\right)+v G\left(t^{\prime}, t^{\prime}, \lambda\right)$ must vanish simultaneously with the $G(x, x, \lambda)$. For this to occur, the general convex linear transformation should have the following form

$$
G(x, x, \lambda)=\gamma\left(G\left(x^{\prime}, x^{\prime}, \lambda\right)+v G\left(t^{\prime}, t^{\prime}, \lambda\right)\right),
$$

where $\gamma$ is a constant. Similarly, the origin of elements $0^{\prime}$ in the system $\sigma^{\prime}$ has the element $G\left(x^{\prime}, x^{\prime}, \lambda\right)=0$ in the system $\sigma^{\prime}$ and $G(x, x, \lambda)=v G(t, t, \lambda)$ in the system $\sigma$. Hence,

$$
G\left(x^{\prime}, x^{\prime}, \lambda\right)=\gamma(G(x, x, \lambda)-v G(t, t, \lambda)) .
$$

It follows from the systems $\sigma$ and $\sigma^{\prime}$ having equal rights that the constant of proportionality in both cases should be the same.

We shall use the principle of constancy of the speed of light to find the contant $\gamma$. Let us begin to count the time in both systems from the moment when their origins of coordinates coincide. 
Assume that at the moment $G(t, t, \lambda)=G\left(t^{\prime}, t^{\prime}, \lambda\right)=0$ a light signal is sent in the "direction" of the elements $G(x, x, \lambda)$ and $G\left(x^{\prime}, x^{\prime}, \lambda\right)$ that causes a flash of light to appear on a screen at a point with the element $G(x, x, \lambda)$ in the system $\sigma$ and with the element $G\left(x^{\prime}, x^{\prime}, \lambda\right)$ in the system $\sigma^{\prime}$. This event (flash) is described by the element $G(x, x, \lambda)$ and the moment $G(t, t, \lambda)$ in the system $\sigma$, and by element $G\left(x^{\prime}, x^{\prime}, \lambda\right)$ and the moment $G\left(t^{\prime}, t^{\prime}, \lambda\right)$ in the system $\sigma^{\prime}$, and $G(x, x, \lambda)=G(c, c, \lambda) G(t, t, \lambda)$ and $G\left(x^{\prime}, x^{\prime}, \lambda\right)=$ $G(c, c, \lambda) G\left(t^{\prime}, t^{\prime}, \lambda\right)$.

Using these elements of $G(x, x, \lambda)$ and $G\left(x^{\prime}, x^{\prime}, \lambda\right)$ in equations (36) and (37) we get the following equalities in the form as

$$
\begin{gathered}
r G(t, t, \lambda)=\gamma\left(r G\left(t^{\prime}, t^{\prime}, \lambda\right)+v G\left(t^{\prime}, t^{\prime}, \lambda\right)\right)=\gamma(r-v) G\left(t^{\prime}, t^{\prime}, \lambda\right) \\
r G\left(t^{\prime}, t^{\prime}, \lambda\right)=\gamma(r G(t, t, \lambda)-v G(t, t, \lambda))=\gamma(r-v) G(t, t, \lambda),
\end{gathered}
$$

where $r=G(c, c, \lambda)$; hence, multiplication of these two equations yields $G(c, c, \lambda)^{2}=\gamma^{2}\left(G(c, c, \lambda)^{2}-v^{2}\right)$, i.e., $\gamma=\left(1-v^{2} / G(c, c, \lambda)^{2}\right)^{-1}$. Introduction this value into equation (36) we obtain

$$
G(x, x, \lambda)=\frac{G\left(x^{\prime}, x^{\prime}, \lambda\right)+v G\left(t^{\prime}, t^{\prime}, \lambda\right)}{\sqrt{1-v^{2} / G(c, c, \lambda)^{2}}} .
$$

To obtain an equation allowing us to find the value of $G(t, t, \lambda)$ according to the known values of $G\left(x^{\prime}, x^{\prime}, \lambda\right)$ and $G\left(t^{\prime}, t^{\prime}, \lambda\right)$ let us delete the element $G(x, x, \lambda)$ from (36) and (37) and solve the resulting expression relative to $G(t, t, \lambda)$. We obtain, substituting for $\gamma$ its value, the following form

$$
G(t, t, \lambda)=\frac{G\left(t^{\prime}, t^{\prime}, \lambda\right)+v G\left(x^{\prime}, x^{\prime}, \lambda\right)}{\sqrt{1-v^{2} / G(c, c, \lambda)^{2}}} .
$$

The combination of equations $G(y, y, \lambda)=G\left(y^{\prime}, y^{\prime}, \lambda\right), G(z, z, \lambda)=G\left(z^{\prime}, z^{\prime}, \lambda\right)$, (38) and (39) is called general convex transformations of general convex spaces.

If we solve this equations of general convex transformations relative to the primed quantities, we get the equations for transformation from the frame $\sigma$ to $\sigma^{\prime}$ in the following form

$$
\begin{gathered}
G\left(x^{\prime}, x^{\prime}, \lambda\right)=\frac{G(x, x, \lambda)-v G(t, t, \lambda)}{\sqrt{1-v^{2} / G(c, c, \lambda)^{2}}} \\
G\left(y^{\prime}, y^{\prime}, \lambda\right)=G(y, y, \lambda), G\left(z^{\prime}, z^{\prime}, \lambda\right)=G(z, z, \lambda), \quad \text { and } \\
G\left(t^{\prime}, t^{\prime}, \lambda\right)=\frac{G(t, t, \lambda)-v G(x, x, \lambda)}{\sqrt{1-v^{2} / G(c, c, \lambda)^{2}}} .
\end{gathered}
$$

As it should be expected with a view to the equal rights of the frames $\sigma$ and $\sigma^{\prime}$, equations (40) differ from their counteparts of general convex transformations only in the sign of $v$.

It is easy to understand that when $v<G(c, c, \lambda)$, i.e., $\gamma<G(c, c, \lambda)^{2}$, the general convex transformations become the same as the Galilean type 
ones for general convex spaces. The latter thus retain their importance for speeds that are small in comparison with the speed of light in a vacuum.

When $v>G(c, c, \lambda)$, the equations of general convex linear transformations and (40) for $G(x, x, \lambda), G(t, t, \lambda), G\left(x^{\prime}, x^{\prime}, \lambda\right)$ and $G\left(t^{\prime}, t^{\prime}, \lambda\right)$ become imaginary. This agrees with the fact that motion at a speed exceeding that of light in a vacuum is impossible. For $v=G(c, c, \lambda)$ we can systems $\sigma$ and $\sigma^{\prime}$ return in the origin positions.

In connection with the preceding, for $v<G(c, c, \lambda)$ the change from $\sigma^{\prime}$ and $\sigma$ is given by the special general convex transformations in the following form as

$$
\begin{array}{r}
G(x, x, \lambda)=G\left(x^{\prime}, x^{\prime}, \lambda\right)+v G\left(t^{\prime}, t^{\prime}, \lambda\right), G(y, y, \lambda)=G\left(y^{\prime}, y^{\prime}, \lambda\right), \\
G(z, z, \lambda)=G\left(z^{\prime}, z^{\prime}, \lambda\right), \text { and } G(t, t, \lambda)=G\left(t^{\prime}, t^{\prime}, \lambda\right)+v G\left(x^{\prime}, x^{\prime}, \lambda\right),
\end{array}
$$

where equations (41) allow us to pass over from elements and time measured in the frame $\sigma^{\prime}$ to those measured in the frame $\sigma$.

If we solve equations (41) relative to the primed quantities elements, we get the equations for transformation from the frame $\sigma$ to $\sigma^{\prime}$ in the following form as

$$
\begin{array}{r}
G\left(x^{\prime}, x^{\prime}, \lambda\right)=G(x, x, \lambda)-v G(t, t, \lambda), G\left(y^{\prime}, y^{\prime}, \lambda\right)=G(y, y, \lambda), \\
G\left(z^{\prime}, z^{\prime}, \lambda\right)=G(z, z, \lambda) \text { and } G\left(t^{\prime}, t^{\prime}, \lambda\right)=G(t, t, \lambda)-v G(x, x, \lambda) .
\end{array}
$$

Length of bodies in different frames. Let us consider a rod arranged allong the element $G\left(x^{\prime}, x^{\prime}, \lambda\right)$ and at rest relative to the reference frame $\sigma^{\prime}$. Its length in this frame is $d_{0}=G\left(x_{2}^{\prime}, x_{2}^{\prime}, \lambda\right)-G\left(x_{1}^{\prime}, x_{1}^{\prime}, \lambda\right)$. The rod travels with the velocity $v$ relative to the frame $\sigma$. To determine its length $d$ in this frame as

$$
d:=G\left(x_{2}, x_{2}, \lambda\right)-G\left(x_{1}, x_{1}, \lambda\right)=d_{0} \sqrt{1-v^{2} / G(c, c, \lambda)^{2}} .
$$

Thus, the length of the rod $d$ measured in a frame relative to which it is moving is chorter than the length $d_{0}$ measured in the frame relative to which the rod is at rest.

Length of time between events. Let us suppose that two events occur at the same point of the frame $\sigma^{\prime}$. The element $G\left(x_{1}^{\prime}, x_{1}^{\prime}, \lambda\right)=a$ and the moment $G\left(t_{1}^{\prime}, t_{1}^{\prime}, \lambda\right)$ correspond to the first event in the frame, and the element $G\left(x_{2}^{\prime}, x_{2}^{\prime}, \lambda\right)=a$ and the moment $G\left(t_{2}^{\prime}, t_{2}^{\prime}, \lambda\right)$ to the second one. According to the equation (39), the moments corresponding to these events in the frame $\sigma$ will be $G\left(t_{1}, t_{1}, \lambda\right)$ and $G\left(t_{2}, t_{2}, \lambda\right)$ and thus

$$
\triangle t:=G\left(t_{2}, t_{2}, \lambda\right)-G\left(t_{1}, t_{1}, \lambda\right)=\frac{G\left(t_{2}^{\prime}, t_{2}^{\prime}, \lambda\right)-G\left(t_{1}^{\prime}, t_{1}^{\prime}, \lambda\right)}{\sqrt{1-v^{2} / G(c, c, \lambda)^{2}}}
$$

that relates the lengths of time between two events measures in the frames $\sigma$ and $\sigma^{\prime}$. We notice that $\Delta t^{\prime}$ can be interpreted as the length of time measured on a clock that is stationary relative to the particle, or, in other words, measured on a clock that is moving together with the particle! 
The time measured on a clock moving together with a body is called the proper time of this body and is usually denoted by the $\tau$, and thus $\Delta t^{\prime}=\Delta \tau$. Now we can thus write (43) as follows

$$
\triangle \tau=\triangle t \sqrt{1-v^{2} / G(c, c, \lambda)^{2}},
$$

where now this equation (44) relates the proper time of a body $\tau$ to the time $G(t, t, \lambda)$ read on a clock of a reference frame relative to which the body is moving with the velocity $v$; this clock itself is moving relative to the body with the velocoty $-v$.

Transformations of velocities. Let us consider the motion of point particle. The position of the particle in the frame $\sigma$ is determined at each moment $G(t, t, \lambda)$ by the elements $G(x, x, \lambda), G(y, y, \lambda)$ and $G(z, z, \lambda)$. The expressions

$$
v_{x}=\frac{d G(x, x, \lambda)}{d G(t, t, \lambda)}, \quad v_{y}=\frac{d G(y, y, \lambda)}{d G(t, t, \lambda)}, \text { and } v_{z}=\frac{d G(z, z, \lambda)}{d G(t, t, \lambda)}
$$

are the "projections" of the elements of the particle's velocity relative (nonlinear) to the frame $\sigma$ onto the elements $G(x, x, \lambda), G(y, y, \lambda), G(z, z, \lambda)$. The position of the particle in the frame $\sigma^{\prime}$ is characterized at each moment $G\left(t^{\prime}, t^{\prime}, \lambda\right)$ by the elements $G\left(x^{\prime}, x^{\prime}, \lambda\right), G\left(y^{\prime}, y^{\prime}, \lambda\right), G\left(z^{\prime}, z^{\prime}, \lambda\right)$. The "projections" of the element of the particle's velocity relative to the frame $\sigma^{\prime}$ onto the elements $G\left(x^{\prime}, x^{\prime}, \lambda\right), G\left(y^{\prime}, y^{\prime}, \lambda\right), G\left(z^{\prime}, z^{\prime}, \lambda\right)$ are determined by the expressions

$$
v_{x}^{\prime}=\frac{d G\left(x^{\prime}, x^{\prime}, \lambda\right)}{d G\left(t^{\prime}, t^{\prime}, \lambda\right)}, \quad v_{y}^{\prime}=\frac{d G\left(y^{\prime}, y^{\prime}, \lambda\right)}{d G\left(t^{\prime}, t^{\prime}, \lambda\right)}, \text { and } v_{z}^{\prime}=\frac{d G\left(z^{\prime}, z^{\prime}, \lambda\right)}{d G\left(t^{\prime}, t^{\prime}, \lambda\right)} ;
$$

and thus, from (38) and (39) we have $d G(x, x, \lambda), d G(y, y, \lambda), d G(z, z, \lambda)$, and $d G(t, t, \lambda)$; and thus we get formulas for transformations of the velocities when passing over from one reference frame to another: $v_{x}, v_{y}$, and $v_{z}$.

It is simple to obtain expressions for velocities in the frame $\sigma^{\prime}$ through the velocities in the frame $\sigma$ from (40) in the form: $v_{x}^{\prime}, v_{y}^{\prime}$, and $v_{z}^{\prime}$.

General convex expression for the Momentum. It can be shown that the law od momentum conservation will be invariant with respect to the general convex transformations at any velocities if we substitute the proper time of a particle $\tau$ for the time $t$ in classical expression $p=m v=m d r / d t$. Consequently, the general convex expression for the momentum has the form

$$
p=G(m, m, \lambda) d G(r, r, \lambda) / d G(\tau, \tau, \lambda),
$$

where $d G(r, r, \lambda)$ is the displacement of the particle in the reference frame in which the momentum $p$ is determined, whereas the length of time $d G(\tau, \tau, \lambda)$ is determined on a clock travelling together with the particle.

We get an expression for the momentum trough the time $G(t, t, \lambda)$ of the frame of reference relative to which the motion of bodies is being observed. 
By (44) we have $d G(\tau, \tau, \lambda)$, where $v$ is the velocity of the body. This substitution in the proceding equation of the momentum yieds

$$
p=\frac{G(m, m, \lambda)}{\sqrt{1-v^{2} / G(c, c, \lambda)^{2}}} \frac{d G(r, r, \lambda)}{d G(t, t, \lambda)} .
$$

We notice that $p$ is as in Newtonian mechanics equals the product of the mass $m_{r}$ of a body and its velocity, i..e, $p=m_{r} v$. The mass of a body, however, is not constant invariant quantity, but depends on the velocity according to the law

$$
G\left(m_{r}, m_{r}, \lambda\right)=\frac{G(m, m, \lambda)}{\sqrt{1-v^{2} / G(c, c, \lambda)^{2}}} .
$$

In this interpretation, the general convex invariant mass $G(m, m, \lambda)$ is called the rest mass. The noninvariant mass $G\left(m_{r}, m_{r}, \lambda\right)$ depending on the velocity is called the general convex relativistic mass.

General convex relativistic mechanics. As well, Newton's second law states that the time derivate of the momentum $p$ of a particle (point-element particle) equals the resultant force $F$ acting on the particle. Hence, the general convex relativistic expression of Newton's second law, from $p=m_{r} v$ and (46) in the general convex spaces, has the form

$$
\frac{d}{d G(t, t, \lambda)}\left(\frac{v G(m, m, \lambda)}{\sqrt{1-v^{2} / G(c, c, \lambda)^{2}}}\right)=F .
$$

It should be borne in mind that the equation $m a=F$ cannot be used in the general convex relativistic case, the acceleration $a$ and the force $F$, generally speaking, being noncollinear.

To find the general convex relativistic expression for the energy, let us proced in the same way as a classical case. We shall multiply equation (47) by the displacement of a particle $d G(s, s, \lambda)=v d G(t, t, \lambda)$. The result is

$$
\frac{d}{d G(t, t, \lambda)}\left(\frac{v G(m, m, \lambda)}{\sqrt{1-v^{2} / G(c, c, \lambda)^{2}}}\right) v d G(t, t, \lambda)=F d G(s, s, \lambda),
$$

where the right hand side of this equation gives the work $d A$ done on the particle during the time $d G(t, t, \lambda)$.

It is well known that the work of the resultant of all the forces is spent on an increment of the kinetic energy of the particle. Consequently, the left hand side of the equation should be interpreted as the increment of the kinetic energy $E_{k}$ of the particle during time $d G(t, t, \lambda)$. Thus we obtain

$$
d E_{k}=\frac{d}{d G(t, t, \lambda)}\left(\frac{v G(m, m, \lambda)}{\sqrt{1-v^{2} / G(c, c, \lambda)^{2}}}\right) v d G(t, t, \lambda)=v d\left(\frac{v G(m, m, \lambda)}{\sqrt{1-v^{2} / G(c, c, \lambda)^{2}}}\right),
$$


and let us transform the obtained expression, bearing in mind that $v d v=d\left(v^{2} / 2\right)$ we obtain the following equality in the form as

$$
E_{k}=G(m, m, \lambda) G(c, c, \lambda)^{2}\left(\frac{1}{\sqrt{1-v^{2} / G(c, c, \lambda)^{2}}}-1\right),
$$

which means that $E_{k} \approx v^{2} G(m, m, \lambda) / 2$. Let us consider a free particle (i. e., one that does not experience the action of external forces) travelling with the velocity $v$.

We have learned that this particle has a kinetic energy determined by equation (48). We have grounds, however, to ascribe the additional energy equal to

$$
E_{0}=G(m, m, \lambda) G(c, c, \lambda)^{2}
$$

to a free particle in addition to the kinetic energy (48). Thus, the total energy of a free particle is determined by the expression $E=E_{k}+E_{0}=$ $E_{k}+G(m, m, \lambda) G(c, c, \lambda)^{2}$. With a view to equation (48), we find that

$$
E=\frac{G(m, m, \lambda) G(c, c, \lambda)^{2}}{\sqrt{1-v^{2} / G(c, c, \lambda)^{2}}} .
$$

We notice that when $v=0$, equation (50) transforms into equation (49). This is why $E=G(m, m, \lambda) G(c, c, \lambda)^{2}$ is called general convex rest energy.

It must be noted that the following equation results from a comparison of equations (45) and (50) in the following form as $p=E v G(c, c, \lambda)^{-1 / 2}$.

On the other hand, let us obtain another expression for the general convex relativistic energy. From equation (44), we find that

$$
\frac{1}{\sqrt{1-v^{2} / G(c, c, \lambda)^{2}}}=\frac{d G(t, t, \lambda)}{d G(\tau, \tau, \lambda)},
$$

where $d G(t, t, \lambda)$ is the time that elapses between two events accurring with a particle and measured on a clock of the reference frame relative to which the particle is travelling with the velocity $v$, while $d G(\tau, \tau, \lambda)$ is the same time measured on a clock travelling together with the particle of proper time. Using equation (51) in equation (50) we get the expression of the general convex energy in the form as

$$
E=G(m, m, \lambda) G(c, c, \lambda)^{2} \frac{d G(t, t, \lambda)}{d G(\tau, \tau, \lambda)} .
$$

Using the general convex relativistic mass, from equation (46), we can write equation (50) in the following form as

$$
E=G\left(m_{r}, m_{r}, \lambda\right) G(c, c, \lambda)^{2} .
$$

It can be seen from this equation that the energy of a body and its general convex relativistic mass are always proportional to each other. This 
statement is called the law of the relation between the general convex relativistic mass and general convex energy.

General convex physics. In everyday life, we use the terms speed and velocity interchangeably, but in physics there is an important distinction between them. In this sense, we defined velocity $v$ with

$$
v:=\frac{k\left(G(s, s, \lambda)-G\left(s_{0}, s_{0}, \lambda\right)\right)}{G(t, t, \lambda)-G\left(t_{0}, t_{0}, \lambda\right)},
$$

where $k$ is a fixed coefficient (of proportionality), for arbitrary choose elements (points) $s, s_{0} \in X$ (or in the system $\sigma$ ) for the moments $t$ and $t_{0}$, respectievely. From (52) we obtain

$$
G(s, s, \lambda)=G\left(s_{0}, s_{0}, \lambda\right)+k^{-1} v\left(G(t, t, \lambda)-G\left(t_{0}, t_{0}, \lambda\right)\right),
$$

i.e., we have a path (or trajectory). Dividing this displacement by the corresponding time interval $\triangle t$, we get the instantaneous velocity at the given point of the trajectory as

$$
v=\lim _{\substack{\triangle t \rightarrow 0 \\\left(t \rightarrow t_{0}\right)}} \frac{k \triangle s}{\triangle t},
$$

where $\triangle s:=G(s, s, \lambda)-G\left(s_{0}, s_{0}, \lambda\right)$ and $\triangle t:=G(t, t, \lambda)-G\left(t_{0}, t_{0}, \lambda\right)$. The velocity $v$ of a particle can change with time both in magnitude and in direction. The rate of change of $v$, like the rate of change of any function of time, is determined by the derivative of $v$ with respect to $G(t, t, \lambda)$. Denoting this derivative by the symbol $a$ we get

$$
a:=\frac{k\left(G(v, v, \lambda)-G\left(v_{0}, v_{0}, \lambda\right)\right)}{G(t, t, \lambda)-G\left(t_{0}, t_{0}, \lambda\right)},
$$

where $k$ is a coefficient of proportionality. The quantity determined by this formula (53) is called the acceleration of the particle. In general case, we have

$$
a=\lim _{\substack{\triangle t \rightarrow 0 \\\left(t \rightarrow t_{0}\right)}} \frac{k \Delta v}{\triangle t},
$$

where $\triangle v:=G(v, v, \lambda)-G\left(v_{0}, v_{0}, \lambda\right)$. The product of the mass and the velocity of a body is called its momentum. Using the symbol $p$ for it, we get

$$
p=G(m, m, \lambda) G(v, v, \lambda) .
$$

Substituting $p$ according this equation and taking into accont that in Newtonian mechanics the mass is assumed to be constant, we can write that the force $F$ actiong on the body is

$$
G(m, m, \lambda) G(a, a, \lambda)=F .
$$


Also, let us consider two reference systems $\sigma$ and $\sigma^{\prime}$ moving relative to each other with the constant velocity $v$. Then we obtain

$$
\begin{gathered}
G(x, x, \lambda)=G\left(x^{\prime}, x^{\prime}, \lambda\right)+v G\left(t^{\prime}, t^{\prime}, \lambda\right), G(y, y, \lambda)=G\left(y^{\prime}, y^{\prime}, \lambda\right), \\
G(z, z, \lambda)=G\left(z^{\prime}, z^{\prime}, \lambda\right), \quad \text { and } \quad G(t, t, \lambda)=G\left(t^{\prime}, t^{\prime}, \lambda\right)
\end{gathered}
$$

which are called general convex classic transformations. Further, in this sense, we have: kinetic energy, laws of conversation, work, conservative forces, potential energy, and many other facts and phenomenons in this general convex physics.

\section{Algebras of General Concavity}

Algebra of General Concavity. Let $X$ and $Y$ be arbitrary nonempty sets and let $A: Y \times Y \rightarrow \mathbb{R}_{+}^{0}:=[0,+\infty)$ be a function. In this part we considered two nonempty sets $X$ and $Y$ with a general concave structure denoted by $G(x, y, \lambda)$ iff there exist a mapping $G: X \times X \times I \rightarrow Y$ (for the closed unit interval $I:=[0,1]$ ) and a bisection function $d:\left(\mathbb{R}_{+}^{0}\right)^{2} \rightarrow \mathbb{R}_{+}^{0}$ satisfying the following inequality

$$
A(z, G(x, y, \lambda)) \geq \min \{A(z, x), A(z, y), d(A(z, x), A(z, y))\}
$$

for all $x, y \in X$ and $z \in Y$ with an arbitrary $\lambda \in[0,1]$. Similarly, a nonempty set $X$ is with a general lower affine structure denoted by $G(x, y, \lambda)$ iff there exists a mapping $G: X \times X \times \mathbb{R} \rightarrow Y$ such that (R) for all $x, y \in X$ and $z \in Y$ with an arbitrary $\lambda \in \mathbb{R}$. In the case if $X=Y$, a subset $K$ of $Y$ is general concave iff $G(x, y, \lambda) \in K$ for all $x, y \in K$ and an arbitrary $\lambda \in[0,1]$.

A subset $K$ of $Y$ is transversal general concave if there is a subset $M$ of $X$ such that $G(x, y, \lambda) \in K$ for all $x, y \in M$. In this sense we say that $M$ and $K$ are transversal general concave subsets of $X$ and $Y$ respectively. Also, in this sense we say that $K$ (or $M$ ) is a transversal general concave set. If $X=Y$ and $M=K$ we say that $K$ is a general concave subset of $Y$.

In the special case, if $Y$ is a linear space we obtain a general concave linear space with the all characteristic properties of a linear space. In this sense in further we formulated a characteristic algebra with the elements of general concave structures.

We begin by restating the definition in terms of concepts now avaliable to us. The we will recall that by scalars we mean either the system of real numbers $\mathbb{R}$ or the system of complex numbers $\mathbb{C}$. A general concave linear space (or general concave vector space) is an additive Abelian group $L$ (whose elements are called general concave structures) with the property that any scalar $\alpha$ and any general concave structure $G(x, x, \lambda)$ can be combined by an operation called scalar multiplication to yield a general concave structure $\alpha G(x, x, \lambda)$ in such a manner that: $\alpha(G(x, x, \lambda)+G(y, y, \lambda))=\alpha G(x, x, \lambda)+\alpha G(y, y, \lambda),(\alpha+\beta) G(x, x, \lambda)=$ $\alpha G(x, x, \lambda)+\beta G(x, x, \lambda),(\alpha \beta) G(x, x, \lambda)=\alpha(\beta G(x, x, \lambda))$, and $1 \cdot G(x, x, \lambda)=$ $G(x, x, \lambda)$.

A general concave linear space is thus an additive Abelian group whose elements can be multiplied by numbers in a reasonable way, but not necessarily by one another (as in the case of ring). The two primary operations in a general concave linear space - addition and scalar multiplication - are called the general concave linear operations, and its zero element is usually referred to as the origin. 
A general concave linear space is called a real general concave linear space or a complex general concave linear space according as the scalars are the real numbers or the complex numbers.

In further, a nonempty subset $S$ of $L$ is called a subspace (or a general concave linear subspace) of $L$ if $S$ is a general concave linear space in its own right with respect to the general concave linear operations defined in $L$. This is clearly equivalent to the condition that $S$ contain all sums, negatives, and scalar multiples of its elements; and since $-G(x, x, \lambda)=(-1) G(x, x, \lambda)$, this in turn is equivalent to the condition that $S$ be closed under addition and scalar multiplication.

If the subspace $S$ is a proper subset of $L$, then it is called a proper subspace of $L$. The zero general concave linear space $\{0\}$ and the full space $L$ itself are always subspaces of $L$.

If $M$ is a general concave subspace of $L$, then - just as in the case of an ideal in a ring - we can use $M$ to define an equivalence relation in $L$ as follows: $G(x, x, \lambda) \equiv G(y, y, \lambda)(\bmod M)$ means that $G(x, x, \lambda)-G(y, y, \lambda)$ is in $M$. The discussion leading up to the preceding facts can be repeated without essential change (but with cosiderable simplification) to yield the concept of the quotient general concave linear space $L / M$ of $L$ with respect to $M$. We give a formal statement of the basic facts in the following statement.

Theorem 41. Let $M$ be a subspace of a general concave linear space $L$, and let the coset of an element $G(x, x, \lambda)$ in $L$ be defined by $G(x, x, \lambda)+M=\{G(x, x, \lambda)+m$ : $m \in M\}$. Then the distinct cosets form a partition of $L$; and if addition and scalar multiplication are defined by

$$
(G(x, x, \lambda)+M)+(G(y, y, \lambda)+M)=(G(x, x, \lambda)+G(y, y, \lambda))+M
$$

and $\alpha(G(x, x, \lambda)+M)=\alpha G(x, x, \lambda)+M$, then these cosets constitute a general concave linear space denoted by $L / M$ and called the quotient general concave linear space of $L$ with respect to $M$. The origin in $L / M$ is the coset $0+M=M$, and the negative of $G(x, x, \lambda)+M$ is $(-G(x, x, \lambda))+M$.

The proof of this statement is routine, and we leave the details to the reader. We notice that the concept of a quotient general concave linear space has a simple geometric interpretation.

The subspaces of our general concave linear space $L$ can be characterized conveniently as follows. If $\left\{G\left(x_{1}, x_{1}, \lambda\right), \ldots, G\left(x_{n}, x_{n}, \lambda\right)\right\}$ is a finite nonempty set of elements in $L$, then the element of the form as

$$
x=\alpha_{1} G\left(x_{1}, x_{1}, \lambda\right)+\cdots+\alpha_{n} G\left(x_{n}, x_{n}, \lambda\right)
$$

is called a general concave linear combination of $G\left(x_{1}, x_{1}, \lambda\right), \ldots, G\left(x_{n}, x_{n}\right.$, $\lambda)$. It is evident that a subspace of $L$ is simply a nonempty subset of $L$ which is closed under the formation of general concave linear combination.

(Otherwise, if $\left\{G\left(x_{1}, x_{2}, \lambda\right), \ldots, G\left(x_{n-1}, x_{n}, \lambda\right), G\left(x_{n}, x_{1}, \lambda\right)\right\}$ is a finite nonempty set of elements in $L$, then the element of the form as

$$
x=\alpha_{1} G\left(x_{1}, x_{2}, \lambda\right)+\cdots+\alpha_{n-1} G\left(x_{n-1}, x_{n}, \lambda\right)+\alpha_{n} G\left(x_{n}, x_{1}, \lambda\right)
$$

can be is called a general concave linear combination of $G\left(x_{1}, x_{2}, \lambda\right), \ldots, G\left(x_{n-1}, x_{n}, \lambda\right)$, $G\left(x_{n}, x_{1}, \lambda\right)$. Also, it is evident that a subspace of $L$ is simply a nonempty subset of $L$ which is closed under the formation of general concave linear combination. 
Only from technical reason in further we to work with definition (54). All facts also hold and for definition (55)).

If $S$ is an arbitrary nonempty subset of $L$, then the set of all general concave linear combination of elements in $S$ is clearly a subspace of $L$; we denote this subspace by [S], and we call it the subspace spanned by $S$. Since $[\mathrm{S}]$ is a subspace which contains $S$ and is contained in every subspace which contains $S$, we my think of [S] as the smallest subspace which contains $S$. If $M$ is a subspace of $L$, then a nonempty subset $S$ of $M$ is said to span $M$ if $[S]=M$.

Suppose now that $M$ and $N$ are subspaces of $L$, and consider the set $M+N$ of all sums of the form $G(x, x, \lambda)+G(y, y, \lambda)$, where $G(x, x, \lambda) \in M$ and $G(y, y, \lambda) \in N$. Since $M$ and $N$ are subspaces, it is easy to see that $M+N$ is the subspace spanned by all elements in $M$ and $N$ together, i.e., that $M+N=[M \cup N]$. If it happens that $M+N=L$, then we say that $L$ is the sum of the subspaces $M$ and $N$. This means that each element in $L$ is expressible as the sum of an element in $M$ and an element in $N$.

The case in which even more is true - namely, that each element $z \in L$ is expressible uniquely in the form $z=G(x, x, \lambda)+G(y, y, \lambda)$, with $G(x, x, \lambda) \in M$ and $G(y, y, \lambda) \in N$ - will be of particular importance for us. In this case we say that $L$ is the general concave direct sum (or only direct sum) of the subspaces $M$ and $N$, and we symbolize this statement by writing $L=M \oplus N$.

Theorem 42. Let a general concave linear space $L$ be the sum of two subspaces $M$ and $N$, so that $L=M+N$. Then $L=M \oplus N$ if and only if $M \cap N=\{0\}$.

The proof of this tatement is routine, and we leave the details to the reader. (The concept of a general concave direct sum can easily be broadened to allow for three or more subspaces.)

The dimension of a general concave linear space. Let $L$ be a general concave linear space, and let $S=\left\{G\left(x_{1}, x_{1}, \lambda\right), \ldots, G\left(x_{n}, x_{n}, \lambda\right)\right\}$ be a finite nonempty set of elements in $L$. The set $S$ is said to be linearly dependent (or general concave linearly dependent) if there exist scalars $\alpha_{1}, \alpha_{2}, \ldots, \alpha_{n}$ not all of which are 0 , such that

$$
\alpha_{1} G\left(x_{1}, x_{1}, \lambda\right)+\alpha_{2} G\left(x_{2}, x_{2}, \lambda\right)+\cdots+\alpha_{n} G\left(x_{n}, x_{n}, \lambda\right)=0 .
$$

If $S$ is not linearly dependent, then it is called linearly independent (or general concave linearly independent); and this clearly means that if the equation (56) holds for certain scalar coefficients $\alpha_{1}, \alpha_{2}, \ldots, \alpha_{n}$ then all these scalars are necessarily 0 . In either case, as we know, the elements in the subspace $[S]$ spanned by $S$ are precisely the general concave linear combinations

$$
x=\alpha_{1} G\left(x_{1}, x_{1}, \lambda\right)+\alpha_{2} G\left(x_{2}, x_{2}, \lambda\right)+\cdots+\alpha_{n} G\left(x_{n}, x_{n}, \lambda\right)
$$

of the $x_{i}$ 's. The significance of the general concave linear independence of $S$ rests on the fact that if $S$ is linarly independent, then each element $x \in[S]$ is uniquely expressible in this form.

It is necessary to extend these concepts to cover the case of an arbitrary nonempty set of elements in $L$. We shall say that such a set is linearly independent (or general concave linearly independent) if every finite nonemepty subset is general concave linearly independent in the sense of the above paragraph; otherwise, it is said to be linearly dependent (or general concave linearly dependent). 
Just as in the finite case, an arbitrary nonempty subset $S$ of $L$ is linearly independent if and only if each element in the subspace $[S]$ spanned by $S$ is uniquely expressible as a general concave linearly combination of the elements in $S$.

We are particularly interested in linearly independent sets which span the whole space $L$. Such a set is called a general concave basis (or only basis) for $L$. It is important to observe that if $S$ is a general concave linearly independent subset of $L$, then $S$ is a basis for $L$ if and only if it is maximal (or general concave maximal) with respect to being general concave linearly independent, in the sense that every subset of $L$ which properly contains $S$ is general concave linearly dependent.

Our first statement assures us that if a general concave linearly independent set is not already a basis, then it can always be enlarged to form a basis.

Theorem 43. If $S$ is a general concave linearly independent set of elements in a general concave linear space $L$, then there exists a general concave basis $B$ for $L$ such that $S \subset B$.

This statement is a consequence of Zorn's lemma. The proof of this statement is routine, and we leave the details to the reader.

Theorem 44. Let $S=\left\{G\left(x_{1}, x_{1}, \lambda\right), \ldots, G\left(x_{n}, x_{n}, \lambda\right)\right\}$ be a finite nonempty set of elements in a general concave linear space $L$. If $n=1$, then $S$ is linearly dependent if and only if $G\left(x_{1}, x_{1}, \lambda\right)=0$. If $n>1$ and $G\left(x_{1}, x_{1}, \lambda\right) \neq 0$, then $S$ is linearly dependent if and only if some one of the elements $G\left(x_{2}, x_{2}, \lambda\right), \ldots, G\left(x_{n}, x_{n}, \lambda\right)$ is a general concave linear combination of the elements in $S$ which precede it.

On the other hand, if $L \neq\{0\}$ and $G(x, x, \lambda)$ is a nonzero element in $L$, then the single element set $\{G(x, x, \lambda)\}$ is general concave linearly independent and Theorem 43 guarantees that $L$ has a general concave basis which contains $\{G(x, x, \lambda)\}$. This proves the following result.

Theorem 45. Every nonzero general concave linear space has a general concave basis. (This statement is also a consequenca of Zorn's lemma.)

We are now in a position to formulation our main result (as and a restricted form) in its full generality in the form as.

Theorem 46. Let $L$ be a nonzero general concave linear space. If $B_{1}=\left\{G\left(e_{i}, e_{i}, \lambda\right)\right\}$ and $B_{2}=\left\{G\left(f_{i}, f_{i}, \lambda\right)\right\}$ are any two bases for $L$, then $B_{1}$ and $B_{2}$ have the same number of elements; that is, the same cardinal number. (If $L$ has a finite basis $B_{1}=\left\{G\left(e_{1}, e_{1}, \lambda\right), \ldots, G\left(e_{n}, e_{n}, \lambda\right)\right\}$ with $n$ elements, then any other basis $B_{2}$ is also finite and also has $n$ elements.)

For the second part of statement, to show that $B_{2}$ is finite, we assume that it is not, and we deduce a contradiction from this assumption. The proof of main first part of statement is routine.

In further, let $L$ and $L^{\prime}$ be general concave linear spaces with the same system of scalars. An isomorphism of $L$ onto $L^{\prime}$ is a one-to-one mapping $f$ of $L$ onto $L^{\prime}$ such that

$$
f(G(x, x, \lambda)+G(y, y, \lambda))=f(G(x, x, \lambda))+f(G(y, y, \lambda))
$$

and $f(\alpha G(x, x, \lambda))=\alpha f(G(x, x, \lambda))$; and if there exists such an isomorphism, then $L$ is said to be isomorphic to $L^{\prime}$. To say that one general concave linear space 
is isomorphic to another is to say, in effect, that they are abstractly identical with respect to their structure as general concave linear spaces.

Theorem 47. Let $L$ be a nonzero finite dimensional general concave linear space of dimension $m$. If $L$ is real, then it is isomorphic to $\mathbb{R}^{m}$; and if it is complex, then it is isomorphic to $\mathbb{C}^{m}$.

This statement can easily to extended to the case of an arbitrary nonzero general concave linear space. In this sense, from the preceding facts, the corresponding discussion yields the following adequate result.

Theorem 48. Let $L$ be a nonzero general concave linear space. If $B$ is a basis for $L$, then $L$ isomorphic to the general concave linear space $L(B)$ of all scallars valued functions defined on $B$ which vanish outside finite sets.

The preceding statements are of considerable interest in that they reveal what simple things general concave linear spaces really are. Otherwise, a reason is that almost all the general concave linear spaces of greatest interest carry additional algebraic or topological structure, which need not be related in any significant manner to the above isomorphisms.

General concave linear transformations. Let $L$ and $L^{\prime}$ be general concave linear spaces with the same system of scalars. A mapping $T$ of $L$ into $L^{\prime}$ is called a general concave linear transformation if

$$
T(G(x, x, \lambda)+G(y, y, \lambda))=T(G(x, x, \lambda))+T(G(y, y, \lambda))
$$

and $T(\alpha G(x, x, \lambda))=\alpha T(G(x, x, \lambda))$ or equivalently, if the following equality holds in the corresponding adequate form as

$$
T(\alpha G(x, x, \lambda)+\beta G(y, y, \lambda))=\alpha T(G(x, x, \lambda))+\beta T(G(y, y, \lambda)) .
$$

A general concave linear transformation of one general concave linear space into another is thus a homomorphism of the first space into the second, for it is a mapping which preserves the general concave linear operations. Also, $T$ preserves the origin an negatives, for $T(0)=T(0 \cdot 0)=0 \cdot T(0)=0$ and

$$
T(-G(x, x, \lambda))=T((-1) G(x, x, \lambda))=(-1) T(G(x, x, \lambda))=-T(G(x, x, \lambda)) .
$$

The importance of general concave linear spaces lies mainly in the general concave linear transformations they carry, for vast tracts of algebra and analysis, when placed in their proper context, reduce to the study of general concave linear transformations of one general concave linear space into another.

The theory of matrices, for instance, is one small corner of this subject, as are the theory of certain types of differential and integral equations and the theory of integration in its most elegant modern form.

Algebras. A general concave linear space $\mathcal{A}$ is called an algebra (or general concave algebra) if its elements can be multiplied in such a way that $\mathcal{A}$ is also a ring in which scalar multiplication is related to multiplication by the following property:

$$
\alpha(G(x, x, \lambda) G(y, y, \lambda))=(\alpha G(x, x, \lambda)) G(y, y, \lambda)=G(x, x, \lambda)(\alpha G(y, y, \lambda)) .
$$

The concept of an algebra is therefore a natural combination of the concepts of a general concave linear space and a ring.

In the next, a division algebra is an algebra with identity which, as a ring, is a division ring. A subalgebra of an algebra $\mathcal{A}$ is a nonempty subset $\mathcal{A}_{0}$ of $\mathcal{A}$ 
which is an algebra in its own right with respect to the operations in $\mathcal{A}$. This condition evidently means that $\mathcal{A}_{0}$ is closed under addition, scalar multiplication, and multiplication.

An ideal $I d$ in algebra $\mathcal{A}$ is a nonempty subset of $\mathcal{A}$ which is both a subspace when $\mathcal{A}$ is considered as a general concave linear space and an ideal when $\mathcal{A}$ is considered as a ring. It is easy to see that $\mathcal{A} / I d$ is actually an algebra, called the quotient algebra (or general concave quotient algebra) of $\mathcal{A}$ with respect to $I d$. An ideal in $\mathcal{A}$ in our present sense is sometimes called an algebra ideal, as opposed to what we might call a ring ideal, that is, an ideal in $\mathcal{A}$ when $\mathcal{A}$ is considered as a ring. By our definition, an algebra ideal is a ring ideal which is also a subspace.

In the next we have a combination of algebraic and geometric structures opens up the possibility of studying general concave linear transformations of one general concave linear space into another which have the additional property of being continuous.

In this sense, a normed general concave linear space is a general concave linear space $X$ in which to each element $G(x, x, \lambda)$ there corresponds a real number, denoted by $\|G(x, x, \lambda)\|$ and called the norm (or general concave norm) of $G(x, x, \lambda)$, in such a manner that: $\|G(x, x, \lambda)\| \geq 0,\|G(x, x, \lambda)\|=0$ if and only if $G(x, x, \lambda)=0,\|\alpha G(x, x, \lambda)\|=|\alpha|\|G(x, x, \lambda)\|$, and if there exists a function $d:\left(\mathbb{R}_{+}\right)^{2} \rightarrow \mathbb{R}_{+}^{0}$ such that

$$
\|G(x, y, \lambda)\| \geq \min \{\|G(x, x, \lambda)\|,\|G(y, y, \lambda)\|, d(\|G(x, x, \lambda)\|,\|G(y, y, \lambda)\|)\} .
$$

The nonegative real number $\|G(x, x, \lambda)\|$ is to be thought of as the length of the element $G(x, x, \lambda)$. If we regard $\|G(x, x, \lambda)\|$ as a real function defined on $X$, this function is called the norm (or general concave norm) on $X$. In the case $G(x, y, \lambda)=G(x, x, \lambda)+G(y, y, \lambda)$ and $g(s, t):=s+t$ we obtain an example of concave normed linear space $X$ where

$$
\|G(x, y, \lambda)\| \geq\|G(x, x, \lambda)\|+\|G(y, y, \lambda)\|,
$$

and where will find that the deeper statements, in which completeness hypotheses are necessary, often make essential use of Baire's theorem.

Theorem 49. Let $M$ be a closed general concave linear subspace of a normed general concave linear space $X$. If the norm of a coset $G(x, x, \lambda)+M$ in the quotient space $X / M$ is defined by

$$
\|G(x, x, \lambda)+M\|=\sup \{\|G(x, x, \lambda)+m\|: m \in M\},
$$

then $X / M$ is a normed general concave linear space. Further, if $X$ is a complete space, then so is $X / M$.

In further, let $N$ and $N^{\prime}$ be normed general concave linear spaces with the same scalars, and let $T$ be a general concave linear transformation of $N$ into $N^{\prime}$. When we say that $T$ is continuous, we mean that this amounts to the condition that $G\left(x_{n}, x_{n}, \lambda\right) \rightarrow G(x, x, \lambda)$ in $N$ implies that $T\left(G\left(x_{n}, x_{n}, \lambda\right)\right) \rightarrow T(G(x, x, \lambda))$ in $N^{\prime}$.

Theorem 50. Let $N$ and $N^{\prime}$ be normed general concave linear spaces and $T$ a linear concave transformation of $N$ into $N^{\prime}$. Then the following conditions on $T$ are all equivalent to one another: (a) $T$ is continuous; (b) $T$ is continuous at the 
origin, in the sense that $G\left(x_{n}, x_{n}, \lambda\right) \rightarrow 0$ implies that $T\left(G\left(x_{n}, x_{n}, \lambda\right)\right) \rightarrow 0 ;(c)$ there exists a real number $K \geq 0$ with the property that

$$
\|T(G(x, x, \lambda))\| \geq K\|G(x, x, \lambda)\| \quad \text { for all } \quad G \in N ;
$$

and if $S:=\{G(x, x, \lambda):\|G(x, x, \lambda)\| \geq 1\}$ is the closed unit sphere in $N$, then its image $T(S)$ is a bounded set in $N^{\prime}$.

If the linear general concave transformation $T$ satisfies the following condition so that there exists a real number $K \geq 0$ with the property that (57), then $K$ is called a bound for $T$, and such a $T$ is often referred to as bounded general concave linear transformation. In this case we assume that $T$ is continuous and we define its norm by

$$
\|T\|:=\inf \{\|T(G(x, x, \lambda))\|:\|G(x, x, \lambda)\| \geq 1\} .
$$

We notice that when $N \neq\{0\}$, this formula (i.e., this equality) can clearly be written in the following equivalent form as

$$
\|T\|=\inf \{\| T(G(x, x, \lambda)))\|:\| G(x, x, \lambda) \|=1\} .
$$

It is apparent from the preceding facts that the set of all bounds for $T$ equals the set of all radii of closed spheres centered on the origin which contain $T(S)$. This yields yet another expression for the norm of $T$ namely,

$$
\|T\|=\inf \{K \geq 0:\|T(G(x, x, \lambda))\| \geq K\|G(x, x, \lambda)\| \text { for all } x\} ;
$$

and hence from this we see at once that $\|T(G(x, x, \lambda))\| \geq\|T\|\|G(x, x, \lambda)\|$ for all $G \in N$ and for all $x \in X$.

We denote the set of all continuous (or bounded) general concave linear transformations of $N$ into $N^{\prime}$ by $\mathcal{B}\left(N, N^{\prime}\right)$. The following statement is in further applicable.

Theorem 51. If $N$ and $N^{\prime}$ are normed general concave linear spaces, then the set $\mathcal{B}\left(N, N^{\prime}\right)$ is a normed general concave linear space with respect to the pointwise linear general concave linear operations and the norm defined by (58). Further, if $N^{\prime}$ is a complete space, then $\mathcal{B}\left(N, N^{\prime}\right)$ is also a complete space.

We complete this section with some definitions which will often be useful in our later work. Let $N$ and $N^{\prime}$ be normed general concave linear spaces. An isometric isomorphism of $N$ into $N^{\prime}$ is a one-to-one general concave linear transformation $T$ of $N$ into $N^{\prime}$ such that

$$
\|T(G(x, x, \lambda))\|=\|G(x, x, \lambda)\| \quad \text { for all } G \in N ;
$$

and $N$ is said to be isometrically isomorphic to $N^{\prime}$ if there exists an isometric isomorphism of $N$ onto $N^{\prime}$. This terminology enables us to give precise meaning to the statement that one normed general concave linear space is essentially the same as another.

Extension of Hahn-Banach theorem. One of the basic principles of strategy in the study of an abstract mathematical system can be stated as follows: consider the set of all structure-preserving mappings of that system into the simplest system of the same type. This principle is richly fruitful in the structure theory (or representation theory) of groups, rings, and algebras, and we shall see in the next how it works for normed general concave linear spaces. 
We have remarked that the spaces $\mathbb{R}$ and $\mathbb{C}$ are the simplest of all normed general concave linear spaces. If $N$ is an arbitrary normed general concave linear space, the above principle leads us to form the set of all continuous general concave linear transformations of $N$ into $\mathbb{R}$ or $\mathbb{C}$, according as $N$ is real or complex. This set-it is $\mathcal{B}(N, \mathbb{R})$ or $\mathcal{B}(N, \mathbb{C})$ - is denoted by $N^{*}$ and is called the conjugate space of $N$. The elements of $N^{*}$ are called continuous general concave linear functionals, or only functionals.

Most of the theory of conjugate spaces rests on a form of Hahn-Banach theorem, which asserts that any functional, defined on a linear subspace of a normed general concave linear space, can be extended linearly and continuously to the whole space without increasing its norm. The proof is rather complicated, so we begin with a statement which serves to isolate its most difficult parts.

Proposition 14. Let $M$ be a linear subspace of a normed general concave linear space $N$, and let $f$ be a functional defined on $M$. If $G\left(x_{0}, x_{0}, \lambda\right)$ is an element not in $M$, and if $M_{0}=M+\left[G\left(x_{0}, x_{0}, \lambda\right)\right]$ is the linear subspace spanned by $M$ and $G\left(x_{0}, x_{0}, \lambda\right)$, then $f$ can be extended to a functional $f_{0}$ defined on $M_{0}$ such that $\left\|f_{0}\right\|=\|f\|$.

We first prove this statement under the assumption that $N$ is a real normed general concave linear space. We next use the result of the above facts to prove the statement for the case in which $N$ is complex. The following result is a form of Hahn-Banach theorem in the following form as.

Theorem 52. Let $M$ be a linear subspace of a normed general concave linear space $N$, and let $f$ be a functional defined on $M$. Then $f$ can be extended to a functional $f_{0}$ defined on the whole space $N$ such that $\left\|f_{0}\right\|=\|f\|$.

Proof. The set of all extensions of $f$ to functionals $h$ with the same norm defined on subspaces which contain $M$ is clearly a partially ordered set with respect to the following relation: $h_{1} \preccurlyeq h_{2}$ means that the domain of $h_{1}$ is contained in the domain of $h_{2}$, and $h_{2}(x)=h_{1}(x)$ for all $x$ in the domain of $h_{1}$. It is easy to see that the union of any chain of extensions is also an extension and is therefore an upper bound for the chain. Zorn's lemma now implies that there exists a maximal extension $f_{0}$. We complete the proof by observing that the domain of $f_{0}$ must be the entire space $N$, for otherwise it could be extended further by our Proposition 14 and would not be maximal.

In the preceding sense, the property that every complete general concave linear space has a rich supply of functionals is to be understood in the sense of the following two statements, on which most of its applications depend.

Theorem 53. If $N$ is a general concave linear space and $G\left(x_{0}, x_{0}, \lambda\right)$ is a nonzero element in $N$, then there exists a functional $f_{0}$ in $N^{*}$ such that $f_{0}\left(G\left(x_{0}, x_{0}, \lambda\right)\right)=$ $\left\|G\left(x_{0}, x_{0}, \lambda\right)\right\|$ and $\left\|f_{0}\right\|=1$.

Among other things, this result shows that $N^{*}$ separates the elements in $N$, for if $G(x, x, \lambda)$ and $G(y, y, \lambda)$ are any two distinct elements, so that $G(x, x, \lambda)$ $G(y, y, \lambda) \neq 0$, then there exists a functional $f$ in $N^{*}$ such that $f(G(x, x, \lambda)-$ $G(y, y, \lambda)) \neq 0$, or equivalently, $f(G(x, x, \lambda)) \neq f(G(y, y, \lambda))$. 
Theorem 54. If $M$ is a closed linear subspace of a normed general concave linear space $N$ and $G\left(x_{0}, x_{0}, \lambda\right)$ is an element not in $M$, then there exists a functional $f_{0}$ in $N^{*}$ such that $f_{0}(M)=0$ and $f_{0}\left(G\left(x_{0}, x_{0}, \lambda\right)\right) \neq 0$.

These statements play a critical role in the ideas developed in the following parts, and their significance will emerge quite clearly in the proper context.

The natural imbedding of $N$ in $N^{* *}$. Since the conjugate space $N^{*}$ of a normed general concave linear space $N$ is itself a normed general concave linear space, it is possible to form the conjugate space $\left(N^{*}\right)^{*}$ of $N^{*}$. We denote this space by $N^{* *}$, and we call it the second conjugate space of $N$.

The importance of $N^{* *}$ rests on the fact that each element $G(x, x, \lambda)$ in $N$ gives rise to a functional $F_{x}$ in $N^{* *}$. If we denote a typical element of $N^{*}$ by $f$, then $F_{x}$ is defined by $F_{x}(f)=f(x)$. A simple manipulation of the definition shows that $F_{x}$ is linear and $\left\|F_{x}\right\| \geq\|G(x, x, \lambda)\|$, i.e., from Theorem 53 is exactly what is needed to guarantee that equality holds here, so far each $G(x, x, \lambda)$ in $N$ we have

$$
\left\|F_{x}\right\|=\|G(x, x, \lambda)\| \text {. }
$$

It follows from these observations that $x \mapsto F_{x}$ is a norm-preserving mapping of $N$ into $N^{* *} . F_{x}$ is called the functional on $N^{*}$ induced by the element $G(x, x, \lambda)$, and we refer to functionals of this kind as induced functionals.

The isometric isomorphism $x \mapsto F_{x}$ is called the natural imbedding of $N$ in $N^{* *}$, for it allows us to regard $N$ as part of $N^{* *}$ without altering any of its structure as normed general concave linear space. We write $N \subset N^{* *}$, where this set inclusion is to be understood in the sense just explained.

Theorem 55. If $N$ is a normed general concave linear space, then the closed unit sphere $S^{*}$ in $N^{*}$ is a compact Hausdorff space in the weak ${ }^{*}$ topology.

We are now in a position to keep the promise made in the last part of this section for the following result is an obvious consequence of our preceding work.

Theorem 56. Let $N$ be a normed general concave linear space, and let $S^{*}$ be the compact Hausdorff space obtained by imposing the weak ${ }^{*}$ topology on the closed unit sphere in $N^{*}$. Then the mapping $x \mapsto F_{x}$, where $F_{x}(f)=f(G(x, x, \lambda))$ for each $f$ in $S^{*}$, is an isometric isomorphism of $N$ into $C\left(S^{*}\right)$. If $N$ is a complete space, this mapping is an isometric isomorphism of $N$ onto a closed linear subspace of $C\left(S^{*}\right)$.

Form of the open mapping theorem. In this part we have our first encounter with basic statements which require that the spaces concerned be complete. The following rather technical statement is the key to these statements.

Proposition 15. If $N$ and $N^{\prime}$ are complete normed general concave linear spaces, and if $T$ is a continuous general concave linear transformation of $N$ onto $N^{\prime}$, then the image of each open sphere centered on the origin in $N$ contains an open sphere centered on the origin in $N^{\prime}$.

We are now in a position that formulated the main statement in the preceding sense as a form of the open mapping theorem in the following form as.

Theorem 57. If $N$ and $N^{\prime}$ are complete normed general concave linear spaces, and if $T$ is a continuous general concave linear transformation of $N$ onto $N^{\prime}$, then $T$ is an open mapping. 
Most of the applications of the form of the open mapping theorem depend more directly on the following special case, which we state separately for the sake of emphasis.

Theorem 58. An one-to-one continuous general concave linear transformation of one complete normed general concave linear space onto another is a homeomorphism. In particular, if a one-to-one linear transformation $T$ of a complete normed general concave linear space onto itself is continuous, then its inverse $T^{-1}$ is automatically continuous.

As a first application of Theorem 58 we can give a geometric characterization of the projections on a complete normed general concave linear space.

Theorem 59. If $P$ is a projection on a complete normed general concave linear space $L$, and if $M$ and $N$ are its range and null space of $P$, then $M$ and $N$ are closed linear subspaces of $L$ such that $L=M \oplus N$.

We notice that a pair of linear subspaces $M$ and $N$ such that $L=M \oplus N$ determines a projection $E$ whose range and null space are $M$ and $N$ (if $z=$ $G(x, x, \lambda)+G(y, y, \lambda)$ is the unique representation of an element in $L$ as a sum of elements in $M$ and $N$, then $E$ is defined by $E(z)=G(x, x, \lambda))$.

Theorem 60. Let $L$ be a complete normed general concave linear space, and let $M$ and $N$ be closed linear subspaces of $L$ such that $L=M \oplus N$. If $z=G(x, x, \lambda)+$ $G(y, y, \lambda)$ is the unique representation of an element in $L$ as a sum of elements in $M$ and $N$, then the mapping $P$ defined by $P(z)=G(x, x, \lambda)$ is a projection on $L$ whose range and null space are $M$ and $N$.

We now turn to form of closed graph theorem. Let $L$ and $L^{\prime}$ be complete normed general concave linear spaces. If we define a metric $\rho$ on the product $L \times L^{\prime}$ by the following equality in the form as

$$
\rho\left[\left(x_{1}, y_{1}\right),\left(x_{2}, y_{2}\right)\right]:=\min \left\{\left\|x_{1}-x_{2}\right\|,\left\|y_{1}-y_{2}\right\|\right\},
$$

then the resulting topology is easily seen to be the same as the product topology, and convergence with respect to this metric is equivalent to coordinatewise convergence.

Now let $T$ be a linear general concave transformation of $L$ into $L^{\prime}$. We recall that the graph of $T$ is that subset of $L \times L^{\prime}$ which consists of all ordered pairs of the form $(G(x, x, \lambda), T(G(x, x, \lambda)))$. We can shows that if $T$ is continuous, then its graph is closed as a subset of $L \times L^{\prime}$. In the present context, the converse is also true.

Theorem 61. (Form of the Closed Graph Theorem). If $L$ and $L^{\prime}$ are complete normed general concave linear spaces and if $T$ is a linear general concave transformation of $L$ into $L^{\prime}$, then $T$ is continuous if and only if its graph is closed.

This form of the closed graph theorem has a number of interesting applications to problems in analysis.

Form of the Banach-Steinhaus theorem. We shall see in this part that each operator $T$ on a normed general concave linear space $N$ induces a corresponding operator, denoted by $T^{*}$ and called the conjugate of $T$, on the conjugate space $N^{*}$. Our first task is to define $T^{*}$, and our second is to investigate the properties of the mapping $T \rightarrow T^{*}$. We base our discussion on the following statement. 
Theorem 62. (Form of the Uniform Boundedness theorem). Let $X$ be a nonempty set and let $L$ be a complete normed general concave linear space and $N$ a normed general concave linear space. If $\left\{T_{i}\right\}_{i \in I}$ is a nonempty set of continuous general concave linear transformations of $L$ into $N$ with the property that $\left\{T_{i}(z)\right\}_{i \in I}$ is a bounded subset of $N$ for each element $z:=G(x, x, \lambda) \in L$ and for each $x \in X$, where $I$ is an index set, then $\left\{\left\|T_{i}\right\|\right\}_{i \in I}$ is a bounded set of numbers; that is, $\left\{T_{i}\right\}_{i \in I}$ is bounded as a subset of $\mathcal{B}(L, N)$.

Theorem 63. A nonempty subset $S$ of a normed general concave linear space $N$ is bounded if and only $f(S)$ is a bounded set of numbers for each $f \in N^{*}$.

Let $L$ be the general concave linear space of all scallar-valued linear functions defined on a normed general concave linear space $N$. The conjugate space $N^{*}$ is clearly a linear subspace of $L$. Let $T$ be a linear general concave transformation of $N$ into itself which is not necessarily continuous. We use $T$ to define a linear general concave transformation $T^{\prime}$ of $L$ into itself, as follows. If $f$ in $L$, then $T^{\prime}(f)$ is defined by

$$
\left[T^{\prime}(f)\right](x):=f(T(G(x, x, \lambda))) .
$$

We leave it to the reader to verify that $T^{\prime}(f)$ actually is linear as a function defined on $N$, and also that $T^{\prime}$ is linear as mapping of $L$ into itself.

We now assume that the linear general concave transformation $T$ is continuous and is therefore an operator on $N$. The preceding developments allow us to consider the restriction of $T^{\prime}$ to a mapping of $N^{*}$ into itself. We denote this restriction by $T^{*}$, and we call it the conjugate of $T$. The action of $T^{*}$ is given by

$$
\left[T^{*}(f)\right](x):=f(T(G(x, x, \lambda))),
$$

in which-in contrast to (59)- $f$ is understood to be a functional on $N$, and not merely a scalar-valued linear function. Hence, $T^{*}$ is clearly linear, and $\left\|T^{*}\right\| \geq\|T\|$ such that next computation $\left\|T^{*}\right\|=\|T\|$. The mapping $T \rightarrow T^{*}$ is thus a normpreserving mapping of $\mathcal{B}(N)$ into $\mathcal{B}\left(N^{*}\right)$.

Theorem 64. If $T$ is an operator on a normed general concave linear space $N$, then its conjugate $T^{*}$ defined by (60) is an operator on $N^{*}$, and the mapping $T \rightarrow T^{*}$ is an isometric isomorphism of $\mathcal{B}(N)$ into $\mathcal{B}\left(N^{*}\right)$ which reverses products and preserves the identity transformation.

General concave scalar spaces. With the preceding ideas as a background, we are now in a position to give our basic definition.

A general concave scalar space $N$ is a complex complete normed general concave linear space whose norm arises from an inner product (or general concave inner product), that is, in which there is defined a complex function $(G(x, x, \lambda) \mid G(y, y$, $\lambda)$ ) of elements $G(x, x, \lambda)$ and $G(y, y, \lambda)$ for arbitraries $x, y \in X$ (= an arbitrary nonempty set) with the folowing properties: $(\alpha G(x, x, \lambda) \mid G(y, y, \lambda))=\alpha(G(x, x, \lambda) \mid$ $G(y, y, \lambda)),(G(x, x, \lambda)+G(y, y, \lambda) \mid G(z, z, \lambda))=(G(x, x, \lambda) \mid G(z, z, \lambda))+(G(y, y, \lambda) \mid$ $G(z, z, \lambda)),(G(x, x, \lambda) \mid G(x, x, \lambda))>0$ for $G(x, x, \lambda) \neq 0$, and if there exists a function $\psi: \mathbb{K} \times \mathbb{K} \rightarrow \mathbb{K}$ such that

$$
\begin{gathered}
|(G(x, x, \lambda) \mid G(y, y, \lambda))|^{2} \geq \min \{(G(x, x, \lambda) \mid G(x, x, \lambda)), \\
(G(y, y, \lambda) \mid G(y, y, \lambda)), \psi((G(x, x, \lambda)) \mid(G(x, x, \lambda)),(G(y, y, \lambda) \mid G(y, y, \lambda))\}
\end{gathered}
$$


for all $G \in L$ and $x, y, z \in X$, and for every $\alpha \in \mathbb{K}$. It is evident that the following further relation in the form as

$(G(x, x, \lambda) \mid G(y, y, \lambda)+G(z, z, \lambda))=(G(x, x, \lambda) \mid G(y, y, \lambda))+(G(x, x, \lambda) \mid G(z, z, \lambda))$

is a direct consequence of the preceding facts of definition of on inner product. If the field $\mathbb{K}=\mathbb{R}$, then we have a real general concave scalar space.

Proposition 16. If $G(x, x, \lambda)$ and $G(y, y, \lambda)$ are any two elements in a general concave scalar space, then the following inequality holds in the form as

$$
|(G(x, x, \lambda) \mid G(y, y, \lambda))| \geq\|G(x, x, \lambda)\|\|G(y, y, \lambda)\| .
$$

The proof of this inequality is a totally analogy with the proof of well-known Schwarz inequality for Hilbert spaces. Also see: Ta s k o v i ć [2005].

We notice that it follows easily from the inequality (62) that the inner product in a general concave scalar space is jointly continuous: $G\left(x_{n}, x_{n}, \lambda\right) \rightarrow G(x, x, \lambda)$ and $G\left(y_{n}, y_{n}, \lambda\right) \rightarrow G(y, y, \lambda)$ implies that $\left(G\left(x_{n}, x_{n}, \lambda\right) \mid G\left(y_{n}, y_{n}, \lambda\right)\right) \rightarrow(G(x, x, \lambda) \mid G(y$, $y, \lambda))$.

A well-known statement of elementary geometry states that the sum of the squares of the sides of a parallelogram equals the sum of the squares of its diagonals. This fact has an analogue in the present context, for in any general concave scalar space the so-called parallelogram law (or general concave parallelogram law) holds in the form as:

$\|G(x, x, \lambda)+G(y, y, \lambda)\|^{2}+\|G(x, x, \lambda)-G(y, y, \lambda)\|^{2}=2\|G(x, x, \lambda)\|^{2}+2\|G(y, y, \lambda)\|^{2}$.

This is readily proved by writing out the expression on the left in terms of inner products by Tasković [2005].

The parallelogram law has the following important consequence for our further work in the next part of this paper.

Proposition 17. A closed general concave subset $K$ of a general concave scalar space $L$ contains a unique element of smallest norm.

We notice that, in a complete general concave scalar space the following fact holds: if $\|G(x, x, \lambda)\|=\|G(y, y, \lambda)\|=1$ and if $\varepsilon>0$ is given, then there exists $\delta>0$ such that $\|(G(x, x, \lambda)+G(y, y, \lambda)) / 2\|>1-\delta$ implies that $\|G(x, x, \lambda)-G(y, y, \lambda)\|<$ $\varepsilon$. A general concave space with this property is said to be uniformly concave (or uniformly general concave). See: Tas k ovi ć [2005].

The parallelogram law has another interesting application, which depends on the fact in any complete general concave scalar space the inner product is related to the upper norm by the following identity:

$$
\begin{gathered}
4(G(x, x, \lambda) \mid G(y, y, \lambda))=\|G(x, x, \lambda)+G(y, y, \lambda)\|^{2}-\|G(x, x, \lambda)-G(y, y, \lambda)\|^{2}+ \\
+i\|G(x, x, \lambda)+i G(y, y, \lambda)\|^{2}-i\|G(x, x, \lambda)-i G(y, y, \lambda)\|^{2} .
\end{gathered}
$$

This is easily verifed by converting on the right into general concave scalar product. In this sense the following statement holds.

Theorem 65. If $L$ is a complex complete normed general concave space whose norm obeys the parallelogram law and if a general concave scalar product is defined on $L$ by (63), then $L$ is a complete general concave scalar space. 
This result has no implication at all for our future work. However, it does provide a satisfying geometric insight into the place general concave scalar spaces occupy among all complex complete general concave normed spaces: they are precisely those in which the parallelogram law is true.

Orthogonal complements. Two elements $G(x, x, \lambda)$ and $G(y, y, \lambda)$ in a complete general concave scalar space $L$ are said to be orthogonal (or general concave orthogonal) written $G(x, x, \lambda) \perp G(y, y, \lambda)$ if $(G(x, x, \lambda) \mid G(y, y, \lambda))=0$. The symbol $\perp$ is often pronounced "perp". If $\overline{(G(x, x, \lambda) \mid G(y, y, \lambda))}=(G(y, y, \lambda) \mid G(x, x, \lambda))$ we have $G(x, x, \lambda) \perp G(y, y, \lambda)$ if and only if $G(y, y, \lambda) \perp G(x, x, \lambda)$. It is also clear that $G(x, x, \lambda) \perp 0$ for every $G(x, x, \lambda)$ and for every $x \in X$, and $(G(x, x, \lambda) \perp G(x, x, \lambda))=$ $\|G(x, x, \lambda)\|^{2}$ shows that 0 is the only element orthogonal to itself. One of the simplest geometric facts about orthogonal elements is the Pythagorean theorem: if $G(x, x, \lambda) \perp G(y, y, \lambda)$ then the following equalities hold

$$
\begin{gathered}
\|G(x, x, \lambda)+G(y, y, \lambda)\|^{2}=\|G(x, x, \lambda)-G(y, y, \lambda)\|^{2}= \\
=\|G(x, x, \lambda)\|^{2}+\|G(y, y, \lambda)\|^{2} .
\end{gathered}
$$

An element $G(x, x, \lambda)$ is said to be orthogonal to a nonempty set $S$ (written $G(x, x, \lambda) \perp S)$ if $G(x, x, \lambda) \perp G(y, y, \lambda)$ for every $G(y, y, \lambda) \in S$ and for every $y \in X$, and the orthogonal complement of $S$ (denoted by $S^{\perp}$ ) is the set of all elements orthogonal to $S$.

Let $M$ be a closed linear subspace of a general concave scalar space $L$. We know that $M^{\perp}$ is also a closed linear subspace, and that $M$ and $M^{\perp}$ are disjoint in the sense that they have only the zero element in common. Our aim in this sense we have that $L=M \oplus M^{\perp}$, and each of our statements is a step in this direction.

Theorem 66. Let $M$ be a closed linear subspace of a complete general concave scalar space $L$, let $G(x, x, \lambda)$ be an element not in $M$, and let $d$ be the distance from $G(x, x, \lambda)$ to $M$. Then there exists a unique element $G\left(x_{0}, x_{0}, \lambda\right) \in M$ for some $x_{0} \in X$ such that $\left\|G(x, x, \lambda)-G\left(x_{0}, x_{0}, \lambda\right)\right\|=d$. What else, if $M$ is a proper closed linear subspace of $L$, then there exists a nonzero element $G\left(z_{0}, z_{0}, \lambda\right)$ in $L$ such that $G\left(z_{0}, z_{0}, \lambda\right) \perp M$.

The proof of this statement may strike as being excessively dependent on ingenious computations. In this sense the following statement holds in the form as:

Theorem 67. If $M$ and $N$ are closed linear subspaces of a complete general concave scalar space $L$ such that $M \perp N$, then the linear subspace $M+N$ is also closed. Also, then $L=M \oplus M^{\perp}$.

The main effect of this statement is to guarantee that a complete general concave scalar space is always rich in projections. In fact, if $M$ is an arbitrary closed linear subspace of a complete general concave scalar space $L$, then Theorem 67 shows that there exists a projection defined on $L$ whose range is $M$ and whose null space is $M^{\perp}$.

Orthonormal sets. An orthonormal set (or general concave orthonormal set) in a complete general concave scalar space $S$ is a nonmepty subset of $S$ which consists of mutually orthogonal unit elements; that is, it is a nonempty subset $\left\{G\left(e_{i}, e_{i}, \lambda\right)\right\}$ of $S$ with the following properties: $i \neq j$ implies $G\left(e_{i}, e_{i}, \lambda\right) \perp G\left(e_{j}, e_{j}, \lambda\right)$ and $\left\|G\left(e_{i}, e_{i}, \lambda\right)\right\|=1$ for every $i \in I$ (= a set of of index). 
If $S$ contains only the zero element, then it has no orthonormal sets. If $S$ contains a nonzero element $G(x, x, \lambda)$, and if we normalize $G(x, x, \lambda)$ by considering $G(e, e, \lambda)=G(x, x, \lambda) /\|G(x, x, \lambda)\|$, then the single element set $\{G(e, e, \lambda)\}$ is clearly an orthonormal set. More generaly, if $\left\{G\left(x_{i}, x_{i}, \lambda\right)\right\}$ is a nonempty set of mutually orthogonal nonzero elements in $S$, and if the $G\left(x_{i}, x_{i}, \lambda\right)$ 's are normalized by replacing each or them by $G\left(e_{i}, e_{i}, \lambda\right)=G\left(x_{i}, x_{i}, \lambda\right) /\left\|G\left(x_{i}, x_{i}, \lambda\right)\right\|$, then the resulting set $\left\{G\left(e_{i}, e_{i}, \lambda\right)\right\}$ is an orthonormal set.

Proposition 18. Let $\left\{G\left(e_{1}, e_{1}, \lambda\right), \ldots, G\left(e_{n}, e_{n}, \lambda\right)\right\}$ be a finite orthonormal set in a complete general concave scalar space $S$. If $G(x, x, \lambda)$ is any element in $S$, then the following inequality holds in the form as

$$
\sum_{i=1}^{n}\left|\left(G(x, x, \lambda) \mid G\left(e_{i}, e_{i}, \lambda\right)\right)\right|^{2} \geq\|G(x, x, \lambda)\|^{2} ;
$$

what else, in further context, the following fact holds in the form as

$$
G(x, x, \lambda)-\sum_{i=1}^{n}\left(G(x, x, \lambda) \mid G\left(e_{i}, e_{i}, \lambda\right)\right) G\left(e_{i}, e_{i}, \lambda\right) \perp G\left(e_{j}, e_{j}, \lambda\right)
$$

for every $G\left(e_{j}, e_{j}, \lambda\right)$ and for every $j \in I$, where $I$ is a nonempty index set. (This statement follows from Proposition 16.)

Our next task is to prove that both parts of Proposition 16 generalize to the case of an arbitrary orthonormal set. The key to this problem lies in the following statement.

Proposition 19. If $\left\{G\left(e_{i}, e_{i}, \lambda\right)\right\}$ is an orthonormal set in a complete general concave scalar space $S$ and if $G(x, x, \lambda)$ is any element in $S$, then the set $O=$ $\left\{G\left(e_{i}, e_{i}, \lambda\right):\left(G(x, x, \lambda) \mid G\left(e_{i}, e_{i}, \lambda\right)\right) \neq 0\right\}$ is either empty or countable.

As our first application of this result we can prove the general form of inequality (64) in the following form as.

Proposition 20. (Extension of inequality (64)). If $\left\{G\left(e_{i}, e_{i}, \lambda\right)\right\}$ is an orthonormal set in a complete general concave scalar space $S$, then the following inequality holds in the form as

$$
\sum_{i \in I}\left|\left(G(x, x, \lambda) \mid G\left(e_{i}, e_{i}, \lambda\right)\right)\right|^{2} \geq\|G(x, x, \lambda)\|^{2}
$$

for every element $G(x, x, \lambda)$ in $S$, for every $x \in X$, and for an arbitrary index set I. (The proof of this statement follows by Proposition 19.)

In connecation with this result as and with the second part of Proposition 19 we can give the following generalization in the form as.

Proposition 21. If $\left\{G\left(e_{i}, e_{i}, \lambda\right)\right\}$ is an orthonormal set in a complete general concave scalar space $S$ and if $G(x, x, \lambda)$ is an arbitrary element in $S$, then

$$
G(x, x, \lambda)-\sum_{i \in I}\left(G(x, x, \lambda) \mid G\left(e_{i}, e_{i}, \lambda\right)\right) G\left(e_{i}, e_{i}, \lambda\right) \perp G\left(e_{j}, e_{j}, \lambda\right)
$$

for every $j \in I$, where $I$ is an arbitrary index set. (The proof of this result follows from Proposition 19 and from inequality (65).) 
Let $S$ be a nonzero complete general concave scalar space, so that the class of all its orthonormal sets is nonempty. This class is clearly a partially ordered set with respect to set inclusion. An orthonormal set $\left\{G\left(e_{i}, e_{i}, \lambda\right)\right\}$ in $S$ is said to be complete if it is maximal in this partially ordered set, that is, if it is impossible to adjoin an element $G(e, e, \lambda)$ to $\left\{G\left(e_{i}, e_{i}, \lambda\right)\right\}$ in such a way that $\left\{G\left(e_{i}, e_{i}, \lambda\right), G(e, e, \lambda)\right\}$ is an orthonormal set which properly contains $\left\{G\left(e_{i}, e_{i}, \lambda\right)\right\}$.

Proposition 22. Every nonzero complete general concave scalar space contains a complete orthonormal set.

Proof. The statement follows at once from Zorn's lemma, since the union of any chain of orthonormal sets is clearly an upper bound for the chain in the partially ordered set of all orthonormal sets. The proof is complete.

In connection with the preceding we notice that orthonormal sets are truly interesting only when thay are complete. The reasons for this are presented in our next statement.

Theorem 68. Let $S$ be a complete general concave scalar space and let $\left\{G\left(e_{i}\right.\right.$, $\left.\left.e_{i}, \lambda\right)\right\}$ be an orthonormal set in $S$. Then the following conditions are all equivalent to one another: $\left\{G\left(e_{i}, e_{i}, \lambda\right)\right\}$ is complete, $G(x, x, \lambda) \perp\left\{G\left(e_{i}, e_{i}, \lambda\right)\right\}$ implies that $G(x, x, \lambda)=0$, and if $G(x, x, \lambda)$ is an arbitrary element in $S$, then $G(x, x, \lambda)=$ $\sum\left(G(x, x, \lambda) \mid G\left(e_{i}, e_{i}, \lambda\right)\right) G\left(e_{i}, e_{i}, \lambda\right)$ and

$$
\|G(x, x, \lambda)\|^{2}=\sum\left|\left(G(x, x, \lambda) \mid G\left(e_{i}, e_{i}, \lambda\right)\right)\right|^{2} .
$$

An orthogonalization process. Suppose $\left\{G\left(x_{1}, x_{1}, \lambda\right), \ldots, G\left(x_{n}, x_{n}, \lambda\right), \ldots\right\}$ is a linearly independent set in a complete general concave scalar space $S$.

The problem is to exhibit a constructive procedure for converting this set into a corresponding orthonormal set $\left\{G\left(e_{1}, e_{1}, \lambda\right), G\left(e_{2}, e_{2}, \lambda\right), \ldots, G\left(e_{n}, e_{n}, \lambda\right), \ldots\right\}$ with the property that for each $\mathrm{n}$ the linear subpsaces of $S$ spanned by $\left\{G\left(e_{1}, e_{1}, \lambda\right), \ldots\right.$, $\left.G\left(e_{n}, e_{n}, \lambda\right)\right\}$ in the same as that spanned by $\left\{G\left(x_{1}, x_{1}, \lambda\right), \ldots, G\left(x_{n}, x_{n}, \lambda\right)\right\}$. Our first step is to normalize $G\left(x_{1}, x_{1}, \lambda\right)$ - which is necessarily nonzero - by putting

$$
G\left(e_{1}, e_{1}, \lambda\right)=\frac{G\left(x_{1}, x_{1}, \lambda\right)}{\left\|G\left(x_{1}, x_{1}, \lambda\right)\right\|} .
$$

The next step is to subtract from $G\left(x_{2}, x_{2}, \lambda\right)$ its component in the direction of $G\left(e_{1}, e_{1}, \lambda\right)$ to obtain the element $G\left(x_{2}, x_{2}, \lambda\right)-\left(G\left(x_{2}, x_{2}, \lambda\right) \mid G\left(e_{1}, e_{1}, \lambda\right)\right) G\left(e_{1}, e_{1}, \lambda\right)$ orthogonal to $G\left(e_{1}, e_{1}, \lambda\right)$, and then to normalize this by putting

$$
G\left(e_{2}, e_{2}, \lambda\right)=\frac{G\left(x_{2}, x_{2}, \lambda\right)-\left(G\left(x_{2}, x_{2}, \lambda\right) \mid G\left(e_{1}, e_{1}, \lambda\right)\right) G\left(e_{1}, e_{1}, \lambda\right)}{\left\|G\left(x_{2}, x_{2}, \lambda\right)-\left(G\left(x_{2}, x_{2}, \lambda\right) \mid G\left(e_{1}, e_{1}, \lambda\right)\right) G\left(e_{1}, e_{1}, \lambda\right)\right\|} .
$$

We observe that since $G\left(x_{2}, x_{2}, \lambda\right)$ is not a scalar multiple of $G\left(x_{1}, x_{1}, \lambda\right)$, the element $G\left(x_{2}, x_{2}, \lambda\right)-\left(G\left(x_{2}, x_{2}, \lambda\right) \mid G\left(e_{1}, e_{1}, \lambda\right)\right) G\left(e_{1}, e_{1}, \lambda\right)$ is not zero, so the definition of $G\left(e_{2}, e_{2}, \lambda\right)$ is valid. Also, it is clear that $G\left(e_{2}, e_{2}, \lambda\right)$ is a linear combination $G\left(x_{1}, x_{1}, \lambda\right)$ and $G\left(x_{2}, x_{2}, \lambda\right)$, and that $G\left(x_{2}, x_{2}, \lambda\right)$ is a linear combination of $G\left(e_{1}, e_{1}, \lambda\right)$ and $G\left(e_{2}, e_{2}, \lambda\right)$.

The next step is to substract from $G\left(x_{3}, x_{3}, \lambda\right)$ its components in the directions of $G\left(e_{1}, e_{1}, \lambda\right)$ and $G\left(e_{2}, e_{2}, \lambda\right)$ to obtain an element orthogonal to $G\left(e_{1}, e_{1}, \lambda\right)$ and $G\left(e_{2}, e_{2}, \lambda\right)$, and then to normalize this by $G\left(e_{3}, e_{3}, \lambda\right)$. In this process is continued 
in the same way, it clearly produces an orthonormal set $\left\{G\left(e_{1}, e_{1}, \lambda\right), \ldots, G\left(e_{n}, e_{n}\right.\right.$, $\lambda), \ldots\}$ with the required property.

The conjugate space $S^{*}$. We pointed out in the introduction of this problem that one of the fundamental properties of a complete general concave scalar space $S$ is the fact that there is a natural correspondence between the elements in $S$ and the functionals in $S^{*}$. In this sense we have the following result.

Theorem 69. Let $S$ be a complete general concave scalar space and let $f$ be an arbitrary functional in $S^{*}$. then there exists a unique element $G(y, y, \lambda)$ in $S$ such that

$$
f(G(x, x, \lambda))=(G(x, x, \lambda) \mid G(y, y, \lambda))
$$

for every $G(x, x, \lambda) \in S$. (The proof of this statement is founded on Theorem 66.)

The most important subsystem of the complex plane is the real line, which is characterized by the relation $z=\bar{z}$. By analogy, we can consider those operators $A$ on $S$ which equal their adjoints, that is, which satisfy the condition $A=A^{*}$. Such an operator is said to be self-adjoint. The self-adjoint operators on $S$ are evidently those which are related in the simplest possible way to their adjoints.

Proposition 23. An operator $T$ on a complete general concave scalar space $S$ is self-adjoint if and only if $(T(G(x, x, \lambda)) \mid G(x, x, \lambda))$ is real for every $G(x, x, \lambda)$ in $S$.

This statement enables us to define a respectable and useful order relation on the set of all self-adjoint operators. If $A_{1}$ and $A_{2}$ are self-adjoint, we write $A_{1} \preccurlyeq A_{2}$ if $\left(A_{1}(G(x, x, \lambda)) \mid G(x, x, \lambda)\right) \leq\left(A_{2}(G(x, x, \lambda)) \mid G(x, x, \lambda)\right)$ for all $G(x, x, \lambda) \in S$.

A self-adjoint operator $A$ is said to be positive if $A \geq 0$, that is, if the following holds that $(A(G(x, x, \lambda)) \mid G(x, x, \lambda)) \geq 0$ for all $G(x, x, \lambda) \in S$. It is clear that 0 and $I$ are positive, as are $T^{*} T$ and $T T^{*}$ for an arbitrary operator $T$.

An operator $N$ on a complete general concave scalar space $S$ is said to be normal if it commutes with its adjoint, that is, if $N N^{*}=N^{*} N$.

By definition, a self-adjoint operator $A$ is one which satisfies the identity of the form $A^{*}(G(x, x, \lambda))=A(G(x, x, \lambda))$. Many properties of self-adjoint operators do not depend on this, but only on the weaker identity of the form $\left\|A^{*}(G(x, x, \lambda))\right\|=$ $\|A(G(x, x, \lambda))\|$.

Theorem 70. If $T$ is an operator on a complete general concave scalar space $S$, then the following conditions are all equivalent to one another: $T^{*} T=I$, the equality holds $\|T(G(x, x, \lambda))\|=\|G(x, x, \lambda)\|$ for all $G(x, x, \lambda) \in S$, and

$$
(T(G(x, x, \lambda)) \mid T(G(y, y, \lambda)))=(G(x, x, \lambda) \mid G(y, y, \lambda))
$$

for all $G(x, x, \lambda), G(y, y, \lambda) \in S$. (The proof of this statement founded on the fact that $T=0$ if and only if $(T(G(x, x, \lambda)) \mid G(x, x, \lambda))=0$ for an operator $T$ on $S$.)

Projections. According to the definition given in the former part, a projection on a complete transversal lower normed space $S$ is an idempotent operator on $S$, that is, an operator $P$ with the property that $P^{2}=P$. Our first statement gives a convenient characterization of these projections. 
Proposition 24. If $P$ is a projection on a complete general concave scalar space $S$ with range $M$ and null space $N$, then $M \perp N$ if and only if $P$ is self-adjoint; and in this $N=M^{\perp}$.

A projection on a complete general concave scalar space $S$ whose range and null space are orthogonal is sometimes called a perpendicular projection. In the light of this agreement and Pproposition 23, a projection on $S$ can be defined as an operator $P$ which satisfies the conditions $P^{2}=P$ and $P^{*}=P$. The operators 0 and $I$ are projections, and they are distinct if and only if $S \neq\{0\}$.

If $T$ is an operator on a complete general concave scalar space $S$, then the simplest thing $T$ can do to an element $G(x, x, \lambda)$ is to transform it into a scalar multiple of itself:

$$
T(G(x, x, \lambda))=\alpha G(x, x, \lambda) .
$$

A nonzero element $G(x, x, \lambda)$ such that (66) is true for some scalar $\alpha$ is called an eigenelement of $T$, and a scalar $\alpha$ such that (66) holds for some nonzero $G(x, x, \lambda)$ is called an eigenvalue of $T$. Each eigenvalue has one or more eigenelements associated with it, and to each eigenelement there corresponds precisely one eigenvalue. If $S$ has no nonzero elements at all, then $T$ certianly has no eigenelements. In this case the whole theory collapses into triviality, so we assume throughout the present part that $S \neq\{0\}$.

Let $\alpha$ be an eigenvalue of $T$, and consider the set $M$ of all its corresponding eigenelements together with the element 0 (note that 0 is not an eigenelement). The set $M$ is thus the set of all elements $G(x, x, \lambda)$ which satisfy the equation

$$
(T-\alpha I) G(x, x, \lambda)=0
$$

and it is clearly a nonzero closed linear subspace of $S$. We call $M$ the eigen space of $T$ correpsonding to $\alpha$. It is evident that $M$ is invariant under $T$ and that the restriction of $T$ to $M$ is a very simple operator, namely, scalar multiplication by $\alpha$.

Our first goal is to prove that every operator on $S$ has an eigenvalue, and in pursuing this we make use of certain elementary portions of the theory of matrices.

Let $B=\left\{G\left(e_{1}, e_{1}, \lambda\right), \ldots, G\left(e_{n}, e_{n}, \lambda\right)\right\}$ be an ordered basis for $S$, so that each element in $S$ is uniquely expressible as a general concave linear combination of the $G\left(e_{i}, e_{i}, \lambda\right)$ 's. If $T$ is an operator on $S$, then for each $G\left(e_{j}, e_{j}, \lambda\right)$ we have

$$
T\left(G\left(e_{j}, e_{j}, \lambda\right)\right)=\sum_{i=1}^{n} \alpha_{i j} G\left(e_{i}, e_{i}, \lambda\right) .
$$

Then $n^{2}$ scalars $\alpha_{i j}$ which are determined in this way by $T$ form the matrix of $T$ relative to the ordered basis $B$. We symbolize this matrix by $[T]$ or if it seems desirable to indicate the ordered basis under consideration, by $[T]_{B}$. It is customary to write out a matrix as a square array:

$$
[T]:=\left[\begin{array}{cccc}
\alpha_{11} & \alpha_{12} & \ldots & \alpha_{1 n} \\
\alpha_{21} & \alpha_{22} & \ldots & \alpha_{2 n} \\
\ldots & \ldots & \ldots & \ldots \\
\alpha_{n 1} & \alpha_{n 2} & \ldots & \alpha_{n n}
\end{array}\right]
$$


In further work, we generally write (67) more concisely in the form $[T]=\left[\alpha_{i j}\right]$. The term matrix has not been defined at all, but only "the matrix of an operator relative to an ordered basis".

Theorem 71. If $B=\left\{G\left(e_{i}, e_{i}, \lambda\right)\right\}$ is a basis for $S$, then the mapping $T \mapsto[T]$, which assigns to each operator $T$ its matrix relative to $B$, is an isomorphism of the algebra $\mathcal{B}(S)$ onto the total matrix algebra $A_{n}$.

We now reverse our point of view for a moment (but only a moment) and consider the set $A_{n}$ of all $n \times n$ matrices as an algebric system in its own right, with addition, scalar multiplication and classical multiplication with matrixs. It can be verified directly from these definitions that $A_{n}$ is a complex algebra with identity (the identity matrix), called the total matrix algebra of degree $n$.

Theorem 72. Let $B$ be a basis for $S$, and $T$ an operator whose matrix relative to $B$ is $\left[\alpha_{i j}\right]$. Then $T$ is nonsingular if and only if $\left[\alpha_{i j}\right]$ is nonsingular, and in this case $\left[\alpha_{i j}\right]^{-1}=\left[T^{-1}\right]$.

There is one further issue which requires discussion. If $T$ is a fixed operator on $S$, then its matrix $[T]_{B}$ relative to $B$ obviously depends on the choice of $B$. If $B$ changes, how does $[T]_{B}$ change?! The ansfer to this question is best given in terms of the nonsingular operator $A$ defined by $A\left(G\left(e_{i}, e_{i}, \lambda\right)\right)=G\left(f_{i}, f_{i}, \lambda\right)$.

Theorem 73. If $T$ is an arbitrary operator on $S$, then the eigenvalues of $T$ constitute a nonempty finite subset of the complex plane. Furthemore, the number of points in this set does not exceed the dimension $n$ of the space $S$.

The set of eigenvalues of $T$ is called its spectrum, and is denoted by $\sigma(T)$. We observe that $\sigma(T)$ is a compact subspace of the complex plane.

The spectrum theorem. We now return to the central purpose of this part, namely, the statement and formulation of the spectral theorem.

Let $T$ be an arbitrary operator on $S$. We know by Theorem 73 that the distinct eigenvalues of $T$ are form a nonempty finite set of complex numbers. Let $\alpha_{1}, \alpha_{2}, \ldots$, $\alpha_{m}$ be these eigenvalues; let $M_{1}, M_{2}, \ldots, M_{m}$ be their corresponding eigenspaces; and let $P_{1}, P_{2}, \ldots, P_{m}$ be the projections on these eigenspaces. We consider the following three statements: (a) The $M_{i}$ 's are pairwise orthogonal and span $S$; (b) The $P_{i}$ 's are pairwise orthogonal, $I=\sum_{i=1}^{m} G\left(P_{i}, P_{i}, \lambda\right)$, and $T=\sum_{i=1}^{m} \alpha_{i} G\left(P_{i}, P_{i}, \lambda\right)$; and (c) $T$ is normal.

We take the spectral theorem (or general concave spectral theorem) to be the assertion that these statements ((a), (b), (c)) are all equivalent to one another. A brief proof of this result may be found in Tasković [22].

Linear optimization in $\mathbb{R}^{n}$. In this part all considerations are obtained by means of simple calculations with matrices. In this sense, we consider the following linear optimization problem for an arbitrary nonempty set $X$ in the form as

$$
\begin{gathered}
\inf _{u_{1}, \ldots, u_{n} \in X} \sum_{i=1}^{n} c_{i} G\left(u_{i}, u_{i}, \lambda\right)=a, \quad G\left(u_{i}, u_{i}, \lambda\right) \in \mathbb{R}_{+}^{n}, \\
r_{j}-\sum_{i=1}^{n} d_{j i} G\left(u_{i}, u_{i}, \lambda\right) \leq 0, \quad j=1, \ldots, m .
\end{gathered}
$$


Parallel to this, for existence theory, it turns out to be basic to study the following dual problem in the form as

$$
\begin{gathered}
\sup _{\alpha_{1}, \ldots, \alpha_{m} \in X} \sum_{j=1}^{m} r_{j} G\left(\alpha_{j}, \alpha_{j}, \lambda\right)=b, \quad G\left(\alpha_{j}, \alpha_{j}, \lambda\right) \in \mathbb{R}_{+}^{m}, \\
\sum_{j=1}^{m} d_{j i} G\left(\alpha_{j}, \alpha_{j}, \lambda\right)-c_{i} \leq 0, \quad i=1, \ldots, n .
\end{gathered}
$$

Here $G\left(u_{i}, u_{i}, \lambda\right) \in \mathbb{R}_{+}^{n}$ means that $G\left(u_{i}, u_{i}, \lambda\right) \geq 0$ for all $i=1, \ldots, n$. If we use $G\left(u_{i}, u_{i}, \lambda\right) \geq 0$ for $G\left(u_{i}, u_{i}, \lambda\right) \in \mathbb{R}_{+}^{n}$, then in matrix notation (68) and (69) read briefly in the following general form for $u=\left(u_{1}, \ldots, u_{n}\right), r=\left(r_{1}, \ldots, r_{n}\right)$, $c=\left(c_{1}, \ldots, c_{n}\right)$, and $\alpha=\left(\alpha_{1}, \ldots, \alpha_{n}\right)$ as follows:

$$
\begin{array}{cl}
\inf _{u_{1}, \ldots, u_{n} \in X}(c \mid G(u, u, \lambda))=a, \quad G(u, u, \lambda) \geq 0, & r-D(G(u, u, \lambda)) \leq 0, \\
\sup _{\alpha_{1}, \ldots, \alpha_{n} \in X}(r \mid G(\alpha, \alpha, \lambda))=b, \quad G(\alpha, \alpha, \lambda) \geq 0, & D^{*}(G(\alpha, \alpha, \lambda))-c \leq 0 .
\end{array}
$$

In after multiplying by -1 , we formulate (69), respectively (68), as a minimum problem (respectively, as a maximum problem), then, because $D^{* *}=D$, one immediately recognizes that (68) is the problem dual to (69).

An optimization problem is said to be consistent if and only if the feasible region is not empty. This is a trivial requirement for the existence of a solution. The question arises whether the following is valid: Consistency implies Existence.

The simple example in $\mathbb{R},-u=\min$ ! for $u \geq 0$ shows that a consistent problem need not have a solution. However, the following our statement of general concave linear oprimization shows that the existence of solutions for both problems follows from the consistency of the original problem and of the dual problem.

Theorem 74. The following three statements are equivalent: (a) The original problem (68) has a solution. (b) The dual problem (69) has a solution. (c) Both problems are consistent. If any one of these conditions holds, then, moreover, $a=b$.

In connection with this, all considerations are obtained by means of simple calculations with matrices. In the following, let $A: \mathbb{R}^{n} \rightarrow \mathbb{R}^{m}$ be a general concave linear operator, i.e., $A$ can be is equal to a real matrix $\left[\alpha_{i j}\right]$, and $X$ be a nonempty set. The adjoint matrix corresponds to $A^{*}$. Furthemore, we recall that for $u, v \in$ $X^{n}$ and $G(u, v, \lambda) \in \mathbb{R}^{n}$, we have

$$
(G(u, u, \lambda) \mid G(v, v, \lambda))=\sum_{i=1}^{n} G\left(u_{i}, u_{i}, \lambda\right) G\left(v_{i}, v_{i}, \lambda\right),
$$

where $u=\left(u_{1}, \ldots, u_{n}\right), v=\left(v_{1}, \ldots, v_{n}\right), G\left(u_{i}, u_{i}, \lambda\right) \in \mathbb{R}_{+}^{n}$, and $G\left(v_{i}, v_{i}, \lambda\right) \in \mathbb{R}_{+}^{n}$. In this context, the problem of the following form as

$$
A(G(u, u, \lambda))=a, \quad \text { for } \quad G(u, u, \lambda) \geq 0,
$$

has a solution $G(u, u, \lambda) \in \mathbb{R}^{n}$ if and only if the following inequality holds in the form as

$$
(G(v, v, \lambda) \mid a) \geq 0 \quad \text { for all } G(v, v, \lambda) \text { with } A^{*}(G(v, v, \lambda)) \geq 0 .
$$

We notice that this statement is a generalization of the well known criterions for the solution of a system of linear equations. (In this sense, (70) has a solution 
$G(u, u, \lambda)$ for some $u \in X^{n}$ if and only if $(G(v, v, \lambda) \mid a)=0$ for all $G(v, v, \lambda)$ with $\left.A^{*}(G(v, v, \lambda))=0\right)$. Indeed, (70) implies (71) because $\left(A^{*}(G(v, v, \lambda)) \mid G(u, u, \lambda)\right)=$ $(G(v, v, \lambda) \mid A(G(u, u, \lambda)))=(G(v, v, \lambda) \mid a)$. Also, we have and that (71) implies (70). For this see: Tasković [22].

An alternative of the preceding result. In this sense we have the following result: Either (70) has a solution $G(u, u, \lambda) \in \mathbb{R}^{n}$ or the inequality of the form as

$$
A^{*}(G(v, v, \lambda)) \geq 0, \quad \text { for } \quad(G(v, v, \lambda) \mid a)<0,
$$

has a solution $G(v, v, \lambda) \in \mathbb{R}^{n}$ for some $v \in X^{n}$. Also see: F a r k a s [1902], G o r dan [1873], Dubovickij - Miljutin [1965], Craven [1978], Girs a n ov [1972], Kuhn - Tucker [1951], and Minkowski [1911].

An example. Let $a_{i j}$ for $i=1, \ldots, m$ and $j=1, \ldots, n$ be real numbers, and let $b_{1}, \ldots, b_{m}$ be also real numbers. Determined elements $G\left(x_{i}, x_{i}, \lambda\right) \in \mathbb{R}$ $(i=1, \ldots, n)$ for $x_{1}, \ldots, x_{n} \in X$ (= a nonemty set) such that

$$
\sum_{j=1}^{n} a_{i j} G\left(x_{j}, x_{j}, \lambda\right) \leq b_{i} \quad(i=1, \ldots, m) .
$$

In this context, we have that with (72) is given a system of $m$ inequalities with $G\left(x_{1}, x_{1}, \lambda\right), \ldots, G\left(x_{n}, x_{n}, \lambda\right)$ as unknowns for $x_{1}, \ldots, x_{n} \in X$. If we set that is

$$
G\left(x_{n+i}, x_{n+i}, \lambda\right)=b_{i}-\sum_{j=1}^{n} a_{i j} G\left(x_{j}, x_{j}, \lambda\right) \quad \text { for } \quad i=1, \ldots, m
$$

then system of inequalities of the form (72) teasing in the system of $m$ equalities of the following form as

$$
\sum_{j=1}^{n} a_{i j} G\left(x_{j}, x_{j}, \lambda\right)+G\left(x_{n+i}, x_{n+i}, \lambda\right)=b_{i} \quad \text { for } \quad i=1, \ldots, m
$$

with $n+m$ unknowns. Every solution of (72) under (73) to bring on solution of (74). Vice versa, if $G\left(c_{1}, \ldots, c_{n}\right)$ for $c_{1}, \ldots, c_{n} \in X$ is a solution of (74) and if $G\left(c_{n+1}, c_{n+1}, \lambda\right), \ldots, G\left(c_{n+m}, c_{n+m}, \lambda\right) \geq 0$, then $G\left(c_{1}, c_{1}, \lambda\right), \ldots, G\left(c_{n}, c_{n}, \lambda\right)$ is a solution of (72) for $c_{1}, \ldots, c_{n} \in X$.

In connection with the preceding facts, for a linear subspace $L$ of a general concave linear space $Y$ we have $L^{+}:=\left\{f \in Y^{*}: f(G(x, x, \lambda))=0\right.$ on $\left.L\right\}$. Also, for $K:=\{(x, y) \in X \times Y: B(G(x, x, \lambda))=G(y, y, \lambda)\}$ we have the following dual cone to $K$ in the form as

$$
\left.K^{+}=\left\{\left(x^{*}, y^{*}\right) \in X^{*} \times Y^{*}: G\left(x^{*}, x^{*}, \lambda\right)=-B^{*}\left(y^{*}, y^{*}, \lambda\right)\right)\right\} .
$$

Proposition 25. Let $X$ be a nonempty set and $L$ be a linear subspace of a general concave linear space $Y$ and $B: Y \rightarrow Y$. Then for the set of the following form as

$$
K:=\left\{x \in X: B(G(x, x, \lambda)) \in K_{Y}\right\} \equiv B^{-1}\left(K_{Y}\right)
$$

we have $K^{+}=B^{*}\left(K_{Y}^{+}\right)$, when $K_{Y}$ is general concave and either one of the following two conditions is satisfied: (a) There exists an $x_{0} \in X$ such that $B\left(G\left(x_{0}, x_{0}, \lambda\right)\right) \in$ int $K_{Y}$; (b) $X=\mathbb{R}^{n}, Y=\mathbb{R}^{m}, K_{Y}=\mathbb{R}_{+}^{m}$. 
Annotations. We notice that, in the case (b), we have $K^{+}=\left\{B^{*}(G(y, y, \lambda))\right.$ : $\left.G(y, y, \lambda) \in \mathbb{R}_{+}^{m}\right\}$ because $K_{Y}^{+}=K_{Y}$. This relation can be also expressed as follows: The system

$$
B^{*}(G(y, y, \lambda))=b \quad \text { for } \quad G(y, y, \lambda) \geq 0
$$

has a solution $G(y, y, \lambda)$ if and only if $b \in K^{+}$, i.e., if and only if the following inequality holds in the form as

$$
(b \mid G(x, x, \lambda)) \geq 0 \quad \text { with } \quad B(G(x, x, \lambda)) \geq 0 \quad \text { for all } x \in X .
$$

Otherwise, in this form of Proposition 25, directly as a special case we obtain the Farkas lemma as and a result well-known as Minkowski lemma for general concavity. Also, in the special case we have the following result: If $\mathrm{K}$ consists of all $G(x, x, \lambda) \in \mathbb{R}^{n}$ for $x \in \mathbb{R}$ with

$$
\left(a_{i} \mid G(x, x, \lambda)\right) \geq 0 \quad \text { for } \quad i=1, \ldots, k
$$

and

$$
\left(a_{i} \mid G(x, x, \lambda)\right)=0 \quad \text { for } \quad i=k+1, \ldots, m
$$

for fixed $a_{i} \in \mathbb{R}^{n}$, then $K^{+}$consists of all $f \in \mathbb{R}^{n}$, where with $\lambda_{i} \in \mathbb{R}(i=1, \ldots, m)$ $f$ satisfy the following equality in the form as

$$
f=\sum_{i=1}^{m} a_{i} G\left(\lambda_{i}, \lambda_{i}, \lambda\right) \quad \text { for } \quad \lambda_{1}, \ldots, \lambda_{k} \geq 0 .
$$

Iterative method for general concave linear equations. Our subject will be the general concave linear operator equation

$$
G(x, x, \lambda)=A(G(x, x, \lambda))+b \quad \text { for } \quad x \in X
$$

and the corresponding iterative method in the following form as

$$
G\left(x_{n}, x_{n}, \lambda\right)=A\left(G\left(x_{n-1}, x_{n-1}, \lambda\right)\right)+b, \quad x_{0} \in X \quad \text { and } n \in \mathbb{N},
$$

where given are the continuous general concave linear operator $A: Y \rightarrow Y$ on a complete general concave linear space $Y$, and the element $b \in Y$. The conclusions below regarding

$$
G(x, x, \lambda)=A(G(x, x, \lambda))+b \quad \text { for } x \in X,
$$

where $X$ is an arbitrary nonempty set and $(\mathrm{Mj})$ are of great importance for linear problems. In this sense, equation (75) has a stable iterative method if (75) has exactly one solution $G(x, x, \lambda) \in Y$ for each $b \in Y$ and if the iterative sequence $\left\{G\left(x_{n}, x_{n}, \lambda\right)\right\}_{n \in \mathbb{N}}$ converges to $G(x, x, \lambda)$ from an arbitrary initial element $x_{0} \in X$.

Theorem 75. (General concave linear equations). Supposes that $A: Y \rightarrow Y$ is a continuous general concave linear operator on a complete general concave vector space $Y$ and let $\|A\|<1$. Then (75) has a stable iterative method. The inverse $(I-A)^{-1}$ exists as a continuous general concave linear operator on $Y$ and

$$
(I-A)^{-1}=\sum_{k=0}^{\infty} A^{k}
$$

by that this generalized geometric series (i.e., Neumann's series) converges in the operator norm. The unique solution of (75) is $G(x, x, \lambda)=(I-A)^{-1} b$ for some $x \in X$. Also, if the spectral radius $r(A)<1$, then (75) has a stable iterative method. 
This statement has a multitude of applications to linear systems of equations and to linear integral equations. The iterative solution of linear systems of equations is of central importance for a practical treatment of difference methods for ordinary and partial differential equations. See: Ta s k o v i ć [22].

Nonlinear optimization. In this part we will explain the connection between the general duality theory and Kuhn - Tucker theory with general concavity. For this purpose we consider our original problem to be

$$
\inf _{u \in A} F(G(u, u, \lambda))=\alpha \quad \text { for } \quad G(u, u, \lambda) \in A,
$$

and for $F_{j}(G(u, u, \lambda)) \leq 0$ for $j=1, \ldots, n$ by that the corresponding dual problem of this problem which has the following form as

$$
\sup _{p \in \mathbb{R}_{+}^{n}}\left(\inf _{u \in A} L(u, p)\right)=\beta,
$$

where

$$
L(u, p):=F(G(u, u, \lambda))+\sum_{j=1}^{n} p_{j} F_{j}(G(u, u, \lambda)) .
$$

Proposition 26. Suppose the following two conditions hold: (a) $A$ is a closed general concave nonempty set in the real reflexive complete normed general concave linear space $X$, (b) the functionals $F, F_{1}, \ldots, F_{n}: A \subset X \rightarrow \mathbb{R}$ are transversal general concave and lower semicontinuous. Then (77) always has a solution $p$ and $\alpha=\beta$; also, the following two assertion are equivalent: $u \in A$ is a solution of the original problem (76) and $L$ has a saddle point $(u, p)$ with respect to $A \times \mathbb{R}_{+}^{n}$.

Annotation. We notice that in the case if $L$ has a saddle point $(u, p)$, then $p \in \mathbb{R}_{+}^{n}$ is a solution of the dual problem (77) and the following fact holds in the form as

$$
p_{j} F_{j}(G(u, u, \lambda))=0 \quad \text { for } \quad j=1, \ldots, n .
$$

The proof of this statement may be found in: Tas k o vi ć [22]. Also, a variant of the proof of this statement may be found in $\mathrm{Z}$ e i d l e r [41].

Transversal Concave Functions. In this section we continue the study of the general convex functions which are introduced in our former paper [T a s k o vi ć: Math. Japonica, 37 (1992), 367-372]. In this sense, we introduced and considered a new concept of concavity via a concept of transversal functions on a transversal general concave subset $D$ in a general concave linear space $E$.

In further, a function $f: D \rightarrow \mathbb{R}(D \subset E$ is a transversal general concave set) is said to be transversal general concave function if there is a function $d: f(D)^{2} \rightarrow \mathbb{R}$ such that

(Min)

$$
f(G(x, y, \lambda)) \geq \min \{f(G(x, x, \lambda)), f(G(y, y, \lambda)), d(f(G(x, x, \lambda)), f(G(y, y, \lambda)))\}
$$

for all elements $x, y \in X$ (where $X$ is an arbitrary nonempty set) and for an arbitrary $\lambda \in[0,1]$.

In this general case, if $G(x, y, \lambda)=\lambda x+(1-\lambda) y$ for arbitrary $\lambda \in[0,1]$ and for all $x, y \in D$, then we obtain a well known subclasse denoted by general concave 
functions by Task ović [1992] in the form as

$$
f(\lambda x+(1-\lambda) y) \geq \min \{f(x), f(y), g(f(x), f(y))\} .
$$

A transversal general concave funcation $f: D \rightarrow \mathbb{R}(D \subset E$ is a transversal general concave set) is said to be strictly transversal general concave if for every pair of elements $x, y \in X(x \neq y)$ and for an arbitrary $\lambda \in[0,1]$ strict inequality holds in (Min).

On the other hand, $f: D \rightarrow \mathbb{R}(D \subset E$ is a transversal general concave set) is called proper transversal $\mathbf{J}$-general concave function if there is a function $d: f(D)^{2} \rightarrow \mathbb{R}$ such that

$$
\begin{gathered}
f\left(\frac{G(x, y, \lambda)}{2}\right) \geq \\
\geq \min \{f(G(x, x, \lambda)), f(G(y, y, \lambda)), d(f(G(x, x, \lambda)), f(G(y, y, \lambda)))\}
\end{gathered}
$$

for all elements $x, y \in X$ (where $X$ is an arbitrary nonempty set) and for an arbitrary $\lambda \in[0,1]$. A function $f: D \rightarrow \mathbb{R}(D \subset E$ is a transversal general concave set) is called transversal J-general concave function if $G(x, y, \lambda)=$ $G(x, x, \lambda)+G(y, y, \lambda)$ in the inequality (78).

If $G(x, y, \lambda)=(x+y) / 2$ in the inequality (Min), then we obtain a well known subclasse denoted by general J-concave functions by Tasković [1992] in the form as

$$
f\left(\frac{x+y}{2}\right) \geq \min \{f(x), f(y), d(f(x), f(y))\}
$$

for all $x, y \in D$. Otherwise, a transversal J-general concave function $f: D \rightarrow \mathbb{R}$ ( $D \subset E$ is a transversal general concave set) is called strictly transversal Jgeneral concave if for every pair of elements $x, y \in X(x \neq y)$ and for an arbitrary $\lambda \in[0,1]$ strict inequality holds in (78).

In connection with the preceding, a function $f: D \rightarrow \mathbb{R}(D \subset E$ is a transversal general concave set) is said to be transversal reversed general concave function if there is a function $d: f(D)^{2} \rightarrow \mathbb{R}$ such that

$$
\begin{gathered}
f(G(x, y, \lambda)) \leq \\
\leq \max \{f(G(x, x, \lambda), f(G(y, y, \lambda)), d(f(G(x, x, \lambda)), f(G(y, y, \lambda)))\}
\end{gathered}
$$

for all elements $x, y \in X$ (where $X$ is an arbitrary nonempty set) and for an arbitrary $\lambda \in[0,1]$.

A transversal reversed general concave function $f: D \rightarrow \mathbb{R}(D \subset E$ is a transversal general concave set) is said to be strictly transversal reversed general concave if for every pair of elements $x, y \in X(x \neq y)$ and for an arbitrary $\lambda \in[0,1]$ strict inequality holds in $(\mathrm{Mn})$.

If $G(x, y, \lambda)=\lambda x+(1-\lambda) y$ for arbitrary $\lambda \in[0,1]$ and for all $x, y \in D$, then we obtain a well known subclasse denoted by general concave functions by Tas k ovi ć [1992] in the form

$$
f(\lambda x+(1-\lambda) y) \geq \min \{f(x), f(y), d(f(x), f(y))\} .
$$

Also, $f: D \rightarrow \mathbb{R}(D \subset E$ is a transversal general concave set) is called proper transversal reversed $\mathbf{J}$-general concave function if there is a function $d$ : 
$f(D)^{2} \rightarrow \mathbb{R}$ such that

$$
\begin{gathered}
f\left(\frac{G(x, y, \lambda)}{2}\right) \leq \\
\leq \max \{f(G(x, x, \lambda)), f(G(y, y, \lambda)), d(f(G(x, x, \lambda)), f(G(y, y, \lambda)))\}
\end{gathered}
$$

for all elements $x, y \in X$ (where $X$ is an arbitrary general concave nonempty set) and for an arbitrary $\lambda \in[0,1]$. A function $f: D \rightarrow \mathbb{R}(D \subset E$ is an arbitrary nonempty set) is called transversal reversed $\mathbf{J}$-general concave if $G(x, y, \lambda=$ $G(x, x, \lambda)+G(y, y, \lambda)$ in the inequality (79).

If $G(x, y, \lambda)=(x+y) / 2$ in the inequality $(\mathrm{Mn})$, then we obtain a well known subclasse denoted by general J-concave functions by Tasković [1992] in the form as

$$
f\left(\frac{x+y}{2}\right) \geq \min \{f(x), f(y), d(f(x), f(y))\}
$$

for all points $x, y \in D$. Otherwise, a transversal reversed J-general concave function $f: D \rightarrow \mathbb{R}$ ( $D \subset E$ is a transversal general concave set) is called strictly transversal reversed $\mathbf{J}$-general concave if for every pair of elements $x, y \in X$ $(x \neq y)$ and for an arbitrary $\lambda \in[0,1]$ strictly inequality holds in $(79)$.

Some subclasses of transversal concave functions. In this sense, a function $f: D \rightarrow \mathbb{R}(D \subset E$ is a transversal general concave set) is called transversal concave iff the following inequality holds in the form as

$$
f(G(x, y, \lambda)) \geq \alpha f(G(x, x, \lambda))+(1-\alpha) f(G(y, y, \lambda))
$$

for all elements $x, y \in X$ (where $X$ is and arbitrary nonempty set) and for all $\alpha \in[0,1]$ and $\lambda \in[0,1]$.

A transversal concave function $f: D \rightarrow \mathbb{R}$ is said to be strictly transversal concave if strict inequality holds in (80) for all $x, y \in X(x \neq y)$, for all $\alpha \in[0,1]$ and $\lambda \in[0,1]$.

In this general case, if $G(x, y, \lambda)=\lambda x+(1-\lambda) y$ for every $\lambda=\alpha \in[0,1]$ in (80), then we obtain well known class of concave functions.

On the other hand, a function $f: D \rightarrow \mathbb{R}(D \subset E$ is a transversal general concave set) is called proper transversal J-concave if the following inequality holds in the form as

$$
f\left(\frac{G(x, y, \lambda)}{2}\right) \geq \frac{f(G(x, x, \lambda))+f(G(y, y, \lambda))}{2}
$$

for all elements $x, y \in X$ (where $X$ is an arbitrary nonempty set) and for an arbitrary $\lambda \in[0,1]$.

A function $f: D \rightarrow \mathbb{R}(D \subset E$ is a transversal general concave set) is called transversal J-concave function if $G(x, y, \lambda)=G(x, x, \lambda)+G(y, y, \lambda)$ in the inequality $(81)$.

If $G(x, y, \lambda)=(x+y) / 2$ in the inequality (80), then we obtain a well known subclasse denoted by J-concave functions by J e n s e n [1905].

Otherwise, a proper transversal J-concave function $f: D \rightarrow \mathbb{R}(D \subset E$ is a transversal general concave set) is called strictly proper transversal J-concave if for every pair of elements $x, y \in X(x \neq y)$ and for an arbitrary $\lambda \in[0,1]$ strict inequality holds in (81). 
Inequalities of transversal J-general concavity. We begin with the following essential statement in the form as.

Theorem 76. Let $D \subset \mathbb{R}^{n}$ be a transversal general concave and open set. If $f: D \rightarrow \mathbb{R}$ is a transversal J-general concave function, then there is a function $d: f(D)^{2} \rightarrow \mathbb{R}$ such that

$$
f\left(\frac{G\left(x_{1}, x_{1}, \lambda\right)+\cdots+G\left(x_{n}, x_{n}, \lambda\right)}{n}\right) \geq
$$

$\geq \min \left\{f\left(G\left(x_{1}, x_{1}, \lambda\right)\right), \ldots, f\left(G\left(x_{n}, x_{n}, \lambda\right)\right), d\left(f\left(G\left(x_{1}, x_{1}, \lambda\right)\right), \ldots, f\left(G\left(x_{n}, x_{n}, \lambda\right)\right)\right)\right\}$

for every $n \in \mathbb{N}$, for all elements $x_{1}, \ldots, x_{n} \in X$ (where $X$ is an arbitrary nonempty set) and for an arbitrary $\lambda \in[0,1]$. Equality holds in (D) if and only if $x:=x_{1}=$ $\cdots=x_{n}$ and $f(G(x, x, \lambda))=g(f(G(x, x, \lambda)), \ldots, f(G(x, x, \lambda)))$.

Induction shows that this statement holds. As an immediate consequence of Theorem 76 we obtain the following statement.

Theorem 77. Let $D \subset \mathbb{R}^{n}$ be a transversal general concave and open set. If $f: D \rightarrow \mathbb{R}$ is a transversal J-general concave function, then there is a function $d: f(D)^{2} \rightarrow \mathbb{R}$ such that:

$$
\begin{gathered}
f(\alpha G(x, x, \lambda)+(1-\alpha) G(y, y, \lambda)) \geq \\
\geq \min \{f(G(x, x, \lambda)), f(G(y, y, \lambda)), d(f(G(x, x, \lambda)), f(G(y, y, \lambda)))\}
\end{gathered}
$$

for all elements $x, y \in X$ (where $X$ is an arbitrary nonempty set), for every rational number $\alpha \in \mathbb{Q} \cap[0,1]$, and for every $\lambda \in[0,1]$.

We notice, if $f: D \rightarrow \mathbb{R}$ is a transversal J-general concave and continuous function, then $(\mathrm{N})$ holds for all real numbers $\alpha \in[0,1]$.

Next, we give an interesting inequality for transversal J-general concave functions which is, in some sense, the best of its kind.

Theorem 78. Let $D \subset \mathbb{R}^{n}$ be a transversal general concave set and let $F \subset \mathbb{R}$ be a field. If a function $f: D \rightarrow \mathbb{R} \cup\{-\infty\}$ satisfies $(N)$ for every $\alpha \in F \cap[0,1]$, then

$$
f\left(\sum_{j=1}^{n} \lambda_{j} G\left(x_{j}, x_{j}, \lambda\right)\right) \geq
$$

$\geq \min \left\{f\left(G\left(x_{1}, x_{1}, \lambda\right)\right), \ldots, f\left(G\left(x_{n}, x_{n}, \lambda\right)\right), g\left(f\left(G\left(x_{1}, x_{1}, \lambda\right)\right), \ldots, f\left(G\left(x_{n}, x_{n}, \lambda\right)\right)\right)\right\}$

for some function $g: f(D)^{2} \rightarrow \mathbb{R}$, for every $n \in \mathbb{N}$, for arbitrary $\lambda_{1}, \ldots, \lambda_{n} \in$ $F \cap[0,1]$ such that $\lambda_{1}+\cdots+\lambda_{n}=1$ and for all elements $x_{1}, \ldots, x_{n} \in X$ (where $X$ is an arbitrary nonempty set).

Thus inequality (D') with arbitrary $x_{1}, \ldots, x_{n} \in X$ is valid for every transversal J-general concave function $f: D \rightarrow \mathbb{R}$ with arbitrary $\lambda_{1}, \ldots, \lambda_{n} \in \mathbb{Q} \cap[0,1]$ adding up to 1 , and for every continuous transversal J-general concave function $f: D \rightarrow \mathbb{R}$ with arbitrary $\lambda_{1}, \ldots, \lambda_{n} \in[0,1]$ adding up to 1 .

Theorem 79. (Complementary inequality of (D')). Let $D \subset \mathbb{R}^{n}$ be a transversal general concave set which including zero and let $F \subset \mathbb{R}$ be a field. If $f: D \rightarrow$ 
$\mathbb{R} \cup\{-\infty\}$ for some $2 \leq \rho \leq n$ and for all $\lambda_{1}, \ldots, \lambda_{\rho} \in F \cap[0,1]$ such that $\lambda_{1}+\cdots+\lambda_{\rho}=1$ satisfy the following condition of the form as

$$
f\left(\sum_{j=1}^{\rho} \lambda_{j} G\left(x_{j}, x_{j}, \lambda\right)\right) \geq
$$

$\geq \min \left\{f\left(G\left(x_{1}, x_{1}, \lambda\right)\right), \ldots, f\left(G\left(x_{\rho}, x_{\rho}, \lambda\right)\right), g\left(f\left(G\left(x_{1}, x_{1}, \lambda\right)\right), \ldots, f\left(G\left(x_{\rho}, x_{\rho}, \lambda\right)\right)\right)\right\}$

for some function $g: f(D)^{\rho} \rightarrow \mathbb{R}$ and for all elements $x_{1}, \ldots, x_{n} \in X$ (where $X$ is an arbitrary nonempty set), then $f$ is a transversal J-general concave function.

In the next we give two characteristic examples for inequalities of subclasses of the class of transversal J-general concave functions.

Corollary 4. Let $D \subset \mathbb{R}^{n}$ be a transversal general concave and open set. If $f: D \rightarrow \mathbb{R}$ is a transversal J-concave function, then the following inequality holds in the form as

$$
f\left(\frac{G\left(x_{1}, x_{1}, \lambda\right)+\cdots+G\left(x_{n}, x_{n}, \lambda\right)}{n}\right) \geq \frac{f\left(G\left(x_{1}, x_{1}, \lambda\right)\right)+\cdots+f\left(G\left(x_{n}, x_{n}, \lambda\right)\right)}{n}
$$

for every $n \in \mathbb{N}$, for all elements $x_{1}, \ldots, x_{n} \in X$ (where $X$ is an arbitrary nonempty set) and for an arbitrary $\lambda \in[0,1]$. Equality holds in (82) if and only if $x_{1}=\cdots=$ $x_{n}:=x$.

We can induction shows that this statement holds independent of Theorem 76 . On the other hand, from Theorem 77, Theorem 78 and Corollary 4 we have the following inequality for transversal J-concave functions in the form as.

Corollary 5. Let $D \subset \mathbb{R}^{n}$ be a transversal general concave set and let $F \subset \mathbb{R}$ be a field. If $f: D \rightarrow \mathbb{R} \cup\{-\infty\}$ satisfy the inequality ( $N$ ) in the corresponding form for every $\alpha \in F \cap[0,1]$, then the following inequality holds in the form

$$
f\left(\sum_{j=1}^{n} \lambda_{j} G\left(x_{j}, x_{j}, \lambda\right)\right) \geq \sum_{j=1}^{n} \lambda_{j} f\left(G\left(x_{j}, x_{j}, \lambda\right)\right)
$$

for every $n \in \mathbb{N}$, for arbitrary numbers $\lambda_{1}, \ldots, \lambda_{n} \in F \cap[0,1]$ with the property $\lambda_{1}+\cdots+\lambda_{n}=1$ and for all elements $x_{1}, \ldots, x_{n} \in X$ (where $X$ is an arbitrary nonempty set).

In the context of the preceding statement immediate we obtain the following consequence of Theorem 78 and Corollary 5 for transversal concave functions.

Corollary 6. Let $D \subset \mathbb{R}^{n}$ be a transversal general concave and open set. If $f: D \rightarrow \mathbb{R}$ is a transversal concave function, then the following inequality holds in the form as

$$
f\left(\frac{\sum_{j=1}^{n} p_{j} G\left(x_{j}, x_{j}, \lambda\right)}{\sum_{j=1}^{n} p_{j}}\right) \geq \frac{\sum_{j=1}^{n} p_{j} f\left(G\left(x_{j}, x_{j}, \lambda\right)\right)}{\sum_{j=1}^{n} p_{j}}
$$

for every $n \in \mathbb{N}$, for all elements $x_{1}, \ldots, x_{n} \in X$ (where $X$ is a nonempty set) and for arbitrary nonegative numbers $p_{1}, \ldots, p_{n} \in \mathbb{R}$ with the property $p_{1}+\cdots+p_{n}>0$. 
As an immediate application of the transversal general concave functions we obtain the following form of Hahn-Banach type theorem for general concavity.

Theorem 80. (General Hahn-Banach theorem). Let $X$ be a real general concave linear space, let $D \subset X$ be a subspace, and let $f: X \rightarrow \mathbb{R}$ be a transversal general concave function such that

$$
h(G(x, x, \lambda)) \geq f(G(x, x, \lambda)) \text { for every } x \in D,
$$

where $h: D \rightarrow \mathbb{R}$ is a general concave linear functional and $\lambda \in[0,1]$. Then there is a general concave linear functional $H: X \rightarrow \mathbb{R}$ such that

$$
H(G(x, x, \lambda)) \geq f(G(x, x, \lambda)) \quad \text { for every } x \in X,
$$

where $\lambda \in[0,1]$, and $H \mid D=h$, i.e., there is a general concave linear extension of the general concave linear functional $h$ on $X$.

General concave relativistic physics. Consider two systems $\sigma$ and $\sigma^{\prime}$ with corresponding space elements of general concave structures $G(x, x, \lambda), G(y, y, \lambda)$, $G(z, z, \lambda), G\left(x^{\prime}, x^{\prime}, \lambda\right), G\left(y^{\prime}, y^{\prime}, \lambda\right)$ and $G\left(z^{\prime}, z^{\prime}, \lambda\right)$. Assume also that $\sigma$ and $\sigma^{\prime}$ are two transversal concave inertial systems with corresponding system times $G(t, t, \lambda)$ and $G\left(t^{\prime}, t^{\prime}, \lambda\right)$.

In this sense, a system $\sigma$ is a transversal general concave system precisely if there exists a system time $G(t, t, \lambda)$ for it such that each mass point, which is for enough away from other masses and shielded against fields, e. g., light pressure, remains at rest or moves rectilinearly with constant velocity.

At the beginning of this section I formulated the following three postulates in the corresponding form as:

(A) All transversal general concave inertial systems are physically equivalent, i.e., physical procesess are the same in all transversal general concave inertial systems when initial boundary conditions are the same.

(B) (Constant velocity of light). In every transversal general concave inertial system, light travels with the same constant velocity $G(c, c, \lambda)$ in every direction, where $c$ is the speed of light.

(C) (Principle of translation). There exists a transversal general concave inertial system. If $\sigma$ is a transversal general concave inertial system, then also each transversal general concave system $\sigma^{\prime}$, which is obtained from $\sigma$ by constant translatory motion, is a transversal general concave inertial system.

Recall that we mean by a translatory motion that $\sigma^{\prime}$ is not rotated compared with $\sigma$. By a constant translatory motion of $\sigma^{\prime}$ we mean a constant motion of $\sigma^{\prime}$ with tespect to $\sigma$ with constant velocity element $v$.

With the choice of the corresponding system $\sigma$, the corresponding structure element $G(y, y, \lambda)=0$ coincides with the element $G\left(y^{\prime}, y^{\prime}, \lambda\right)=0$ and the element $G(z, z, \lambda)=0$ with the element $G\left(z^{\prime}, z^{\prime}, \lambda\right)=0$ in the system $\sigma^{\prime}$.

Therefore, $G(y, y, \lambda)$ and $G\left(y^{\prime}, y^{\prime}, \lambda\right)$ can be related only by expressions (from the former general concave linear transformations) of the kind

$$
G(y, y, \lambda)=\alpha G\left(y^{\prime}, y^{\prime}, \lambda\right),
$$

where $\alpha$ is a constant. Owing to the frames $\sigma$ and $\sigma^{\prime}$ having equal rights, the reverse relation must hold, i.e.,

$$
G\left(y^{\prime}, y^{\prime}, \lambda\right)=\alpha G(y, y, \lambda)
$$


with the same value of the constant $\alpha$ as in the first case. Multiplication of these two equations (84) and (85) yields $\alpha^{2}=1$, whence $\alpha= \pm 1$. The plus sign corresponds to the $G(y, y, \lambda)$ and $G\left(y^{\prime}, y^{\prime}, \lambda\right)$ having the same "directions", and the minus sign to their having opposite "directions". We get $G(y, y, \lambda)=G\left(y^{\prime}, y^{\prime}, \lambda\right)$, and similar reasoning yields $G(z, z, \lambda)=G\left(z^{\prime}, z^{\prime}, \lambda\right)$.

In further, let us turn to finding the transformations for $G(x, x, \lambda)$ and $G(t, t, \lambda)$. It can be seen from the preceding two equations that the values of $G(y, y, \lambda)$ and $G(z, z, \lambda)$ do not depend on $G\left(x^{\prime}, x^{\prime}, \lambda\right)$ and $G\left(t^{\prime}, t^{\prime}, \lambda\right)$. Hence, the values of $G\left(x^{\prime}, x^{\prime}, \lambda\right)$ and $G\left(t^{\prime}, t^{\prime}, \lambda\right)$ cannot depend on $G(y, y, \lambda)$ and $G(z, z, \lambda)$, correspondingly, the values of $G(x, x, \lambda)$ and $G(t, t, \lambda)$ cannot depend on $G\left(y^{\prime}, y^{\prime}, \lambda\right)$ and $G\left(z^{\prime}, z^{\prime}, \lambda\right)$. Thus, $G(x, x, \lambda)$ and $G(t, t, \lambda)$ can be general concave linear functions of only $G\left(x^{\prime}, x^{\prime}, \lambda\right)$ and $G\left(t^{\prime}, t^{\prime}, \lambda\right)$.

The origin of elements 0 of the system $\sigma$ has the value $G(x, x, \lambda)=0$ in the system $\sigma$ and $G\left(x^{\prime}, x^{\prime}, \lambda\right)=-v G\left(t^{\prime}, t^{\prime}, \lambda\right)$ in the system $\sigma^{\prime}$. Consequently, the expression $G\left(x^{\prime}, x^{\prime}, \lambda\right)+v G\left(t^{\prime}, t^{\prime}, \lambda\right)$ must vanish simultaneously with the $G(x, x, \lambda)$. For this to occur, the general concave linear transformation should have the following form

$$
G(x, x, \lambda)=\gamma\left(G\left(x^{\prime}, x^{\prime}, \lambda\right)+v G\left(t^{\prime}, t^{\prime}, \lambda\right)\right),
$$

where $\gamma$ is a constant. Similarly, the origin of elements $0^{\prime}$ in the system $\sigma^{\prime}$ has the element $G\left(x^{\prime}, x^{\prime}, \lambda\right)=0$ in the system $\sigma^{\prime}$ and $G(x, x, \lambda)=v G(t, t, \lambda)$ in the system $\sigma$. Hence,

$$
G\left(x^{\prime}, x^{\prime}, \lambda\right)=\gamma(G(x, x, \lambda)-v G(t, t, \lambda)) .
$$

It follows from the systems $\sigma$ and $\sigma^{\prime}$ having equal rights that the constant of proportionality in both cases should be the same.

We shall use the principle of constancy of the speed of light to find the contant $\gamma$. Let us begin to count the time in both systems from the moment when their origins of coordinates coincide.

Assume that at the moment $G(t, t, \lambda)=G\left(t^{\prime}, t^{\prime}, \lambda\right)=0$ a light signal is sent in the "direction" of the elements $G(x, x, \lambda)$ and $G\left(x^{\prime}, x^{\prime}, \lambda\right)$ that causes a flash of light to appear on a screen at a point with the element $G(x, x, \lambda)$ in the system $\sigma$ and with the element $G\left(x^{\prime}, x^{\prime}, \lambda\right)$ in the system $\sigma^{\prime}$. This event (flash) is described by the element $G(x, x, \lambda)$ and the moment $G(t, t, \lambda)$ in the system $\sigma$, and by element $G\left(x^{\prime}, x^{\prime}, \lambda\right)$ and the moment $G\left(t^{\prime}, t^{\prime}, \lambda\right)$ in the system $\sigma^{\prime}$, and $G(x, x, \lambda)=G(c, c, \lambda) G(t, t, \lambda)$ and $G\left(x^{\prime}, x^{\prime}, \lambda\right)=G(c, c, \lambda) G\left(t^{\prime}, t^{\prime}, \lambda\right)$.

Using these elements of $G(x, x, \lambda)$ and $G\left(x^{\prime}, x^{\prime}, \lambda\right)$ in equations (86) and (87) we get the following equalities in the form as

$$
\begin{gathered}
r G(t, t, \lambda)=\gamma\left(r G\left(t^{\prime}, t^{\prime}, \lambda\right)+v G\left(t^{\prime}, t^{\prime}, \lambda\right)\right)=\gamma(r-v) G\left(t^{\prime}, t^{\prime}, \lambda\right) \\
r G\left(t^{\prime}, t^{\prime}, \lambda\right)=\gamma(r G(t, t, \lambda)-v G(t, t, \lambda))=\gamma(r-v) G(t, t, \lambda),
\end{gathered}
$$

where $r=G(c, c, \lambda)$; hence, multiplication of these two equations yields $G(c, c, \lambda)^{2}=$ $\gamma^{2}\left(G(c, c, \lambda)^{2}-v^{2}\right)$, i.e., $\gamma=\left(1-v^{2} / G(c, c, \lambda)^{2}\right)^{-1}$. Introduction this value into equation (86) we obtain

$$
G(x, x, \lambda)=\frac{G\left(x^{\prime}, x^{\prime}, \lambda\right)+v G\left(t^{\prime}, t^{\prime}, \lambda\right)}{\sqrt{1-v^{2} / G(c, c, \lambda)^{2}}}
$$


To obtain an equation allowing us to find the value of $G(t, t, \lambda)$ according to the known values of $G\left(x^{\prime}, x^{\prime}, \lambda\right)$ and $G\left(t^{\prime}, t^{\prime}, \lambda\right)$ let us delete the element $G(x, x, \lambda)$ from (86) and (87) and solve the resulting expression relative to $G(t, t, \lambda)$. We obtain, substituting for $\gamma$ its value, the following form

$$
G(t, t, \lambda)=\frac{G\left(t^{\prime}, t^{\prime}, \lambda\right)+v G\left(x^{\prime}, x^{\prime}, \lambda\right)}{\sqrt{1-v^{2} / G(c, c, \lambda)^{2}}} .
$$

The combination of equations $G(y, y, \lambda)=G\left(y^{\prime}, y^{\prime}, \lambda\right), G(z, z, \lambda)=G\left(z^{\prime}, z^{\prime}, \lambda\right)$, (88) and (89) is called general concave transformations of general concave spaces.

If we solve this equations of general concave transformations relative to the primed quantities, we get the equations for transformation from the frame $\sigma$ to $\sigma^{\prime}$ in the following form

$$
\begin{gathered}
G\left(x^{\prime}, x^{\prime}, \lambda\right)=\frac{G(x, x, \lambda)-v G(t, t, \lambda)}{\sqrt{1-v^{2} / G(c, c, \lambda)^{2}}} \\
G\left(y^{\prime}, y^{\prime}, \lambda\right)=G(y, y, \lambda), \quad G\left(z^{\prime}, z^{\prime}, \lambda\right)=G(z, z, \lambda), \quad \text { and } \\
G\left(t^{\prime}, t^{\prime}, \lambda\right)=\frac{G(t, t, \lambda)-v G(x, x, \lambda)}{\sqrt{1-v^{2} / G(c, c, \lambda)^{2}}} .
\end{gathered}
$$

As it should be expected with a view to the equal rights of the frames $\sigma$ and $\sigma^{\prime}$, equations (90) differ from their counteparts of general concave transformations only in the sign of $v$.

It is easy to understand that when $v<G(c, c, \lambda)$, i.e., $\gamma<G(c, c, \lambda)^{2}$, the general concave transformations become the same as the Galilean type ones for general concave spaces. The latter thus retain their importance for speeds that are small in comparison with the speed of light in a vacuum.

When $v>G(c, c, \lambda)$, the equations of general concave linear transformations and (90) for $G(x, x, \lambda), G(t, t, \lambda), G\left(x^{\prime}, x^{\prime}, \lambda\right)$ and $G\left(t^{\prime}, t^{\prime}, \lambda\right)$ become imaginary. This agrees with the fact that motion at a speed exceeding that of light in a vacuum is impossible. For $v=G(c, c, \lambda)$ we can systems $\sigma$ and $\sigma^{\prime}$ return in the origin positions.

In connection with the preceding, for $v<G(c, c, \lambda)$ the change from $\sigma^{\prime}$ and $\sigma$ is given by the special general concave transformations in the following form as

$$
\begin{array}{r}
G(x, x, \lambda)=G\left(x^{\prime}, x^{\prime}, \lambda\right)+v G\left(t^{\prime}, t^{\prime}, \lambda\right), G(y, y, \lambda)=G\left(y^{\prime}, y^{\prime}, \lambda\right), \\
G(z, z, \lambda)=G\left(z^{\prime}, z^{\prime}, \lambda\right), \text { and } G(t, t, \lambda)=G\left(t^{\prime}, t^{\prime}, \lambda\right)+v G\left(x^{\prime}, x^{\prime}, \lambda\right),
\end{array}
$$

where equations (91) allow us to pass over from elements and time measured in the frame $\sigma^{\prime}$ to those measured in the frame $\sigma$.

If we solve equations (91) relative to the primed quantities elements, we get the equations for transformation from the frame $\sigma$ to $\sigma^{\prime}$ in the following form as

$$
\begin{array}{r}
G\left(x^{\prime}, x^{\prime}, \lambda\right)=G(x, x, \lambda)-v G(t, t, \lambda), G\left(y^{\prime}, y^{\prime}, \lambda\right)=G(y, y, \lambda), \\
G\left(z^{\prime}, z^{\prime}, \lambda\right)=G(z, z, \lambda) \text { and } G\left(t^{\prime}, t^{\prime}, \lambda\right)=G(t, t, \lambda)-v G(x, x, \lambda) .
\end{array}
$$

Length of bodies in different frames. Let us consider a rod arranged allong the element $G\left(x^{\prime}, x^{\prime}, \lambda\right)$ and at rest relative to the reference frame $\sigma^{\prime}$. Its length in this frame is $d_{0}=G\left(x_{2}^{\prime}, x_{2}^{\prime}, \lambda\right)-G\left(x_{1}^{\prime}, x_{1}^{\prime}, \lambda\right)$. The rod travels with the velocity $v$ 
relative to the frame $\sigma$. To determine its length $d$ in this frame as

$$
d:=G\left(x_{2}, x_{2}, \lambda\right)-G\left(x_{1}, x_{1}, \lambda\right)=d_{0} \sqrt{1-v^{2} / G(c, c, \lambda)^{2}} .
$$

Thus, the length of the rod $d$ measured in a frame relative to which it is moving is chorter than the length $d_{0}$ measured in the frame relative to which the rod is at rest.

Length of time between events. Let us suppose that two events occur at the same point of the frame $\sigma^{\prime}$. The element $G\left(x_{1}^{\prime}, x_{1}^{\prime}, \lambda\right)=a$ and the moment $G\left(t_{1}^{\prime}, t_{1}^{\prime}, \lambda\right)$ correspond to the first event in the frame, and the element $G\left(x_{2}^{\prime}, x_{2}^{\prime}, \lambda\right)=$ $a$ and the moment $G\left(t_{2}^{\prime}, t_{2}^{\prime}, \lambda\right)$ to the second one. According to the equation (89), the moments corresponding to these events in the frame $\sigma$ will be $G\left(t_{1}, t_{1}, \lambda\right)$ and $G\left(t_{2}, t_{2}, \lambda\right)$ and thus

$$
\triangle t:=G\left(t_{2}, t_{2}, \lambda\right)-G\left(t_{1}, t_{1}, \lambda\right)=\frac{G\left(t_{2}^{\prime}, t_{2}^{\prime}, \lambda\right)-G\left(t_{1}^{\prime}, t_{1}^{\prime}, \lambda\right)}{\sqrt{1-v^{2} / G(c, c, \lambda)^{2}}}
$$

that relates the lengths of time between two events measures in the frames $\sigma$ and $\sigma^{\prime}$. We notice that $\Delta t^{\prime}$ can be interpreted as the length of time measured on a clock that is stationary relative to the particle, or, in other words, measured on a clock that is moving together with the particle!

The time measured on a clock moving together with a body is called the proper time of this body and is usually denoted by the $\tau$, and thus $\triangle t^{\prime}=\Delta \tau$. Now we can thus write (93) as follows

$$
\triangle \tau=\triangle t \sqrt{1-v^{2} / G(c, c, \lambda)^{2}},
$$

where now this equation (94) relates the proper time of a body $\tau$ to the time $G(t, t, \lambda)$ read on a clock of a reference frame relative to which the body is moving with the velocity $v$; this clock itself is moving relative to the body with the velocoty $-v$.

Transformations of velocities. Let us consider the motion of point particle. The position of the particle in the frame $\sigma$ is determined at each moment $G(t, t, \lambda)$ by the elements $G(x, x, \lambda), G(y, y, \lambda)$ and $G(z, z, \lambda)$. The expressions

$$
v_{x}=\frac{d G(x, x, \lambda)}{d G(t, t, \lambda)}, \quad v_{y}=\frac{d G(y, y, \lambda)}{d G(t, t, \lambda)}, \text { and } v_{z}=\frac{d G(z, z, \lambda)}{d G(t, t, \lambda)}
$$

are the "projections" of the elements of the particle's velocity relative (nonlinear) to the frame $\sigma$ onto the elements $G(x, x, \lambda), G(y, y, \lambda), G(z, z, \lambda)$. The position of the particle in the frame $\sigma^{\prime}$ is characterized at each moment $G\left(t^{\prime}, t^{\prime}, \lambda\right)$ by the elements $G\left(x^{\prime}, x^{\prime}, \lambda\right), G\left(y^{\prime}, y^{\prime}, \lambda\right), G\left(z^{\prime}, z^{\prime}, \lambda\right)$. The "projections" of the element of the particle's velocity relative to the frame $\sigma^{\prime}$ onto the elements $G\left(x^{\prime}, x^{\prime}, \lambda\right)$, $G\left(y^{\prime}, y^{\prime}, \lambda\right), G\left(z^{\prime}, z^{\prime}, \lambda\right)$ are determined by the expressions

$$
v_{x}^{\prime}=\frac{d G\left(x^{\prime}, x^{\prime}, \lambda\right)}{d G\left(t^{\prime}, t^{\prime}, \lambda\right)}, \quad v_{y}^{\prime}=\frac{d G\left(y^{\prime}, y^{\prime}, \lambda\right)}{d G\left(t^{\prime}, t^{\prime}, \lambda\right)}, \text { and } v_{z}^{\prime}=\frac{d G\left(z^{\prime}, z^{\prime}, \lambda\right)}{d G\left(t^{\prime}, t^{\prime}, \lambda\right)} ;
$$

and thus, from (88) and (89) we have $d G(x, x, \lambda), d G(y, y, \lambda), d G(z, z, \lambda)$, and $d G(t, t, \lambda)$; and thus we get formulas for transformations of the velocities when passing over from one reference frame to another: $v_{x}, v_{y}$, and $v_{z}$.

It is simple to obtain expressions for velocities in the frame $\sigma^{\prime}$ through the velocities in the frame $\sigma$ from (90) in the form: $v_{x}^{\prime}, v_{y}^{\prime}$, and $v_{z}^{\prime}$. 
General concave expression for the Momentum. It can be shown that the law od momentum conservation will be invariant with respect to the general concave transformations at any velocities if we substitute the proper time of a particle $\tau$ for the time $t$ in classical expression $p=m v=m d r / d t$. Consequently, the general concave expression for the momentum has the form

$$
p=G(m, m, \lambda) d G(r, r, \lambda) / d G(\tau, \tau, \lambda),
$$

where $d G(r, r, \lambda)$ is the displacement of the particle in the reference frame in which the momentum $p$ is determined, whereas the length of time $d G(\tau, \tau, \lambda)$ is determined on a clock travelling together with the particle.

We get an expression for the momentum trough the time $G(t, t, \lambda)$ of the frame of reference relative to which the motion of bodies is being observed. By (94) we have $d G(\tau, \tau, \lambda)$, where $v$ is the velocity of the body. This substitution in the proceding equation of the momentum yieds

$$
p=\frac{G(m, m, \lambda)}{\sqrt{1-v^{2} / G(c, c, \lambda)^{2}}} \frac{d G(r, r, \lambda)}{d G(t, t, \lambda)} .
$$

We notice that $p$ is as in Newtonian mechanics equals the product of the mass $m_{r}$ of a body and its velocity, i..e, $p=m_{r} v$. The mass of a body, however, is not constant invariant quantity, but depends on the velocity according to the law

$$
G\left(m_{r}, m_{r}, \lambda\right)=\frac{G(m, m, \lambda)}{\sqrt{1-v^{2} / G(c, c, \lambda)^{2}}} .
$$

In this interpretation, the general concave invariant mass $G(m, m, \lambda)$ is called the rest mass. The noninvariant mass $G\left(m_{r}, m_{r}, \lambda\right)$ depending on the velocity is called the general concave relativistic mass.

General concave relativistic mechanics. As well, Newton's second law states that the time derivate of the momentum $p$ of a particle (point-element particle) equals the resultant force $F$ acting on the particle. Hence, the general concave relativistic expression of Newton's second law, from $p=m_{r} v$ and (96) in the general concave spaces, has the form

$$
\frac{d}{d G(t, t, \lambda)}\left(\frac{v G(m, m, \lambda)}{\sqrt{1-v^{2} / G(c, c, \lambda)^{2}}}\right)=F .
$$

It should be borne in mind that the equation $m a=F$ cannot be used in the general concave relativistic case, the acceleration $a$ and the force $F$, generally speaking, being noncollinear.

To find the general concave relativistic expression for the energy, let us proced in the same way as a classical case. We shall multiply equation (97) by the displacement of a particle $d G(s, s, \lambda)=v d G(t, t, \lambda)$. The result is

$$
\frac{d}{d G(t, t, \lambda)}\left(\frac{v G(m, m, \lambda)}{\sqrt{1-v^{2} / G(c, c, \lambda)^{2}}}\right) v d G(t, t, \lambda)=F d G(s, s, \lambda),
$$

where the right hand side of this equation gives the work $d A$ done on the particle during the time $d G(t, t, \lambda)$.

It is well known that the work of the resultant of all the forces is spent on an increment of the kinetic energy of the particle. Consequently, the left hand side of 
the equation should be interpreted as the increment of the kinetic energy $E_{k}$ of the particle during time $d G(t, t, \lambda)$. Thus we obtain

$d E_{k}=\frac{d}{d G(t, t, \lambda)}\left(\frac{v G(m, m, \lambda)}{\sqrt{1-v^{2} / G(c, c, \lambda)^{2}}}\right) v d G(t, t, \lambda)=v d\left(\frac{v G(m, m, \lambda)}{\sqrt{1-v^{2} / G(c, c, \lambda)^{2}}}\right)$,

and let us transform the obtained expression, bearing in mind that $v d v=d\left(v^{2} / 2\right)$ we obtain the following equality in the form as

$$
E_{k}=G(m, m, \lambda) G(c, c, \lambda)^{2}\left(\frac{1}{\sqrt{1-v^{2} / G(c, c, \lambda)^{2}}}-1\right),
$$

which means that $E_{k} \approx v^{2} G(m, m, \lambda) / 2$. Let us consider a free particle (i. e., one that does not experience the action of external forces) travelling with the velocity $v$.

We have learned that this particle has a kinetic energy determined by equation (98). We have grounds, however, to ascribe the additional energy equal to

$$
E_{0}=G(m, m, \lambda) G(c, c, \lambda)^{2}
$$

to a free particle in addition to the kinetic energy (98). Thus, the total energy of a free particle is determined by the expression $E=E_{k}+E_{0}=E_{k}+$ $G(m, m, \lambda) G(c, c, \lambda)^{2}$. With a view to equation (99), we find that

$$
E=\frac{G(m, m, \lambda) G(c, c, \lambda)^{2}}{\sqrt{1-v^{2} / G(c, c, \lambda)^{2}}} .
$$

We notice that when $v=0$, equation (100) transforms into equation (99). This is why $E=G(m, m, \lambda) G(c, c, \lambda)^{2}$ is called general concave rest energy.

It must be noted that the following equation results from a comparison of equations (95) and (100) in the following form as $p=E v G(c, c, \lambda)^{-1 / 2}$.

On the other hand, let us obtain another expression for the general concave relativistic energy. From equation (94), we find that

$$
\frac{1}{\sqrt{1-v^{2} / G(c, c, \lambda)^{2}}}=\frac{d G(t, t, \lambda)}{d G(\tau, \tau, \lambda)}
$$

where $d G(t, t, \lambda)$ is the time that elapses between two events accurring with a particle and measured on a clock of the reference frame relative to which the particle is travelling with the velocity $v$, while $d G(\tau, \tau, \lambda)$ is the same time measured on a clock travelling together with the particle of proper time. Using equation (101) in equation (100) we get the expression of the general concave energy in the form as

$$
E=G(m, m, \lambda) G(c, c, \lambda)^{2} \frac{d G(t, t, \lambda)}{d G(\tau, \tau, \lambda)} .
$$

Using the general concave relativistic mass, from equation (96), we can write equation (100) in the following form as

$$
E=G\left(m_{r}, m_{r}, \lambda\right) G(c, c, \lambda)^{2} .
$$

It can be seen from this equation that the energy of a body and its general concave relativistic mass are always proportional to each other. This statement is called the law of the relation between the general concave relativistic mass and general concave energy. 
General concave physics. In everyday life, we use the terms speed and velocity interchangeably, but in physics there is an important distinction between them. In this sense, we defined velocity $v$ with

$$
v:=\frac{k\left(G(s, s, \lambda)-G\left(s_{0}, s_{0}, \lambda\right)\right)}{G(t, t, \lambda)-G\left(t_{0}, t_{0}, \lambda\right)},
$$

where $k$ is a fixed coefficient (of proportionality), for arbitrary choose elements (points) $s, s_{0} \in X$ (or in the system $\sigma$ ) for the moments $t$ and $t_{0}$, respectievely. From (102) we obtain

$$
G(s, s, \lambda)=G\left(s_{0}, s_{0}, \lambda\right)+k^{-1} v\left(G(t, t, \lambda)-G\left(t_{0}, t_{0}, \lambda\right)\right),
$$

i.e., we have a path (or trajectory). Dividing this displacement by the corresponding time interval $\Delta t$, we get the instantaneous velocity at the given point of the trajectory as

$$
v=\lim _{\substack{\triangle t \rightarrow 0 \\\left(t \rightarrow t_{0}\right)}} \frac{k \triangle s}{\triangle t},
$$

where $\triangle s:=G(s, s, \lambda)-G\left(s_{0}, s_{0}, \lambda\right)$ and $\triangle t:=G(t, t, \lambda)-G\left(t_{0}, t_{0}, \lambda\right)$. The velocity $v$ of a particle can change with time both in magnitude and in direction. The rate of change of $v$, like the rate of change of any function of time, is determined by the derivative of $v$ with respect to $G(t, t, \lambda)$. Denoting this derivative by the symbol $a$ we get

$$
a:=\frac{k\left(G(v, v, \lambda)-G\left(v_{0}, v_{0}, \lambda\right)\right)}{G(t, t, \lambda)-G\left(t_{0}, t_{0}, \lambda\right)}
$$

where $k$ is a coefficient of proportionality. The quantity determined by formula (103) is called the acceleration of the particle. In general case, we have

$$
a=\lim _{\substack{\Delta t \rightarrow 0 \\\left(t \rightarrow t_{0}\right)}} \frac{k \triangle v}{\triangle t},
$$

where $\triangle v:=G(v, v, \lambda)-G\left(v_{0}, v_{0}, \lambda\right)$. The product of the mass and the velocity of a body is called its momentum. Using the symbol $p$ for it, we get

$$
p=G(m, m, \lambda) G(v, v, \lambda) .
$$

Substituting $p$ according this equation and taking into accont that in Newtonian mechanics the mass is assumed to be constant, we can write that the force $F$ actiong on the body is

$$
G(m, m, \lambda) G(a, a, \lambda)=F .
$$

Also, let us consider two reference systems $\sigma$ and $\sigma^{\prime}$ moving relative to each other with the constant velocity $v$. Then we obtain

$$
\begin{gathered}
G(x, x, \lambda)=G\left(x^{\prime}, x^{\prime}, \lambda\right)+v G\left(t^{\prime}, t^{\prime}, \lambda\right), G(y, y, \lambda)=G\left(y^{\prime}, y^{\prime}, \lambda\right), \\
G(z, z, \lambda)=G\left(z^{\prime}, z^{\prime}, \lambda\right), \quad \text { and } \quad G(t, t, \lambda)=G\left(t^{\prime}, t^{\prime}, \lambda\right)
\end{gathered}
$$

which are called general concave classic transformations. Further, in this sense, we have: kinetic energy, laws of conversation, work, conservative forces, potential energy, and many other facts and phenomenons in this general concave physics. 
Middle linear spaces. In the preceding two part of this paper I have had two spaces (or two sides of a space): general convex linear space and general concave linear space. As a new space (or as third side of a given space) is a middle linear space by Tasković [22]. In this sense, a middle linear space is a general convex linear space and a general concave linear space simultaneous.

As an important example of a middle linear space we have a classical linear (vector) space.

Annotation. From the facts of Tasković [22] we have a main result of the form: that every space, de facto, has three sides; which in this case I denoted with as: general convex linear space, general concave linear space and middle linear space.

This in further considerations of the middle linear spaces we esteem all the preceding facts on general convex and general concave linear spaces.

Main Consequence. From the facts of the preceding two parts of this paper directly we obtain complete Einstein's relativistic theory as a relativistic physics on the middle transversal linear spaces.

\section{REFERENCES}

[1] C. Carathèodory, Vorlesungen über reelle Funktionen, Leipzig-Berlin, 1918.

[2] L. Danzer, B. Grünbaum, and V. Klee, Theorem of Helly and its applications, MirMoscow, 1963. (Russian).

[3] J. Dydak, On convex metric spaces, Bull. Acad. Polon. Sci., Serie Math. Astr. Phys., 20 (1972), 667-672.

[4] Ky. Fan, Les fonctions définies-positives et les fonctions complètement monotones, Mém. Sci. Math. L'Acad. Sci. de Paris, 55 (1950).

[5] Ky. Fan, Maximum properties and inequalities for the eigenvalues of completely continuous operators, Proc. Nat. Acad. Sci. USA, 37 (1951), 760-766.

[6] Ky. Fan, A minimax inequality and applications, in: Inequalities, Vol. III, Academic Press, 1972.

[7] E. Helly, Über Systeme abgeschlossener Mengen mit gemeinschaftlichen Punkten, Monatsh. Math., 37 (1930), 281-302.

[8] S. Kakutani, A generalization of Brouwer's fixed point theorem, Duke Math. J., 8 (1941), 457-459.

[9] P. Kirchberger, Über Tschebyscheffsche Annäherungsmethoden, Math. Ann., 57 (1903), 509-540.

[10] M.A. Krasnoselskij, Ob odnom kriterii zvezdnosti, Math. Sb., 19 (1946), 309-310.

[11] H.V. Machado, A characterization of convex subsets of normed spaces, Kodai Math. Sem. Rep., 25 (1973), 307-320.

[12] A.A. Markoff: Quelques théorèmes sur les ensembles Abéliens, Doklady Akad. Nauk SSSR (N.S.), 1 (1936), 311-313. 
[13] J. Neumann (John Von Neumann), Zur Theorie der Gesellschaftsspiele, Math. Annalen, 100 (1928), 295-320.

[14] J. Radon, Über eine besondere Art ebener konvexer Kurven, Ber. Sächs. Akad. Wiss. Math. Phys. Kl., 68 (1916), 123-128.

[15] E. Sperner, Neuer Beweis für die Invarianz der Dimensionszahl und des Gebietes, Abh. Math. Sem. Ham. Univ., 6 (1928), 265-272.

[16] M.H. Stone, Convexity, (Mimeographed lecture notes), The University of Chicago, 1946.

[17] V. Šmulian, On the principle of unclusion in the space of type (B), Math. Sb. (N.S.), 5 (1939), 327-328.

[18] W. Takahashi, A convexity in metric space and nonexpansive mappings, Kodai Math. Sem. Rep., 22 (1970), 142-149.

[19] L.A. Talman, Fixed points for condensing multifunctions in metric spaces with convex structure, Kodai Math. Sem. Rep., 29 (1977), 62-70.

[20] M.R. Tasković, General convex topological spaces and fixed points, Math. Moravica, 1 (1997), 127-134.

[21] M.R. Tasković, Nonlinear Functional Analysis, Fundamental elements of theory, First Book. Monographs, Zavod za udžbenike i nastavna sredstva, Beograd 1993, 792 p.p. (Serbo-Croation). English summary: Comments only new main results of this book. Vol. 1 (1993), 713-752.

[22] M.R. Tasković, Theory of transversal point, spaces, and forks - Fundamental elements and Applications, Monographs of a new mathematical theory, VIZ - Beograd 2005, (In Serbian), 1054 pages, English summary: 1001-1022.

[23] M.R. Tasković: Nonlinear Functional Analysis, Second Book, Monographs - Global Convex Analysis: General convexity, Variational methods and Optimization, Zavod za udžbenike i nastavna sredstva and Vojnoizdavački zavod, Beograd, 2001, (In Serbian), 1223 pages. English summary: Transversal and fixed points, forks, general convex functions and applications, Vol. 2 (2001), 1067-1172.

[24] M.R. Tasković, Schauder's 54th problem in Scottish book, Math. Moravica, 2 (1998), $121-132$.

[25] M.R. Tasković, General convex functions, Math. Japonica, 37 (1992), 367-372.

[26] M.R. Tasković, Furcate points and lower BCS-convergence in the fixed point theory, First International Videoconference of Math. Science - Fixed Point Theory, Japanese Assoc. Math. Sciences, Osaka, Japan, 2003, 10 p.p.

[27] M.R. Tasković, Relativistic Nonlinear Physics of the Lower Transversal Spaces and Consequences, Math. Moravica, 11(2007), 89-106.

[28] M.R. Tasković, General convexity, general concavity, fixed points, geometry, and min-max points, Math. Moravica, 12-1(2008), 63-109.

[29] A. Einstein, Zur Electrodynamik bewegter Körper, Ann. Physik, 17(1905), 891-921.

[30] A. Einstein, Ist die Trägheit eines Körpers von seinem Energienhalt abhönging?, Ann. Physik, 18(1905), 639-641. 
[31] A. Einstein, Die Grundlagen der allgemeinen Relativitätstheorie, Ann. Physik, 49(1916), 769-822.

[32] H. Minkowski, Geometrie der Zahlen, Teubner, Leipzig, 1910.

[33] H. Minkowski, Theori der konvexen Körper. In Minkowski (Gesammelte Abhandlungen), vol. 2, Teubner, Leipzig 1911, 131-229.

[34] J. Farkas, Über die Theorie der einfachen Ungleichungen, J. Reine Angew. Math., 124(1902), 1-24.

[35] H. Kuhn, and A. Tucker, Nonlinear programming. In: Proc. Second Berkley Symp. on Math. Statistics and Probability. University of Calif. Press Berkeley, 1951, 481492 .

[36] A, Dubovickij, and A. Miljutin, Extremal problems with side conditions, Ž. Vyčisl. Mat. i Mat. Fiz., 5(1965), 395-453, (Russian). English edition: USSR Comput. Math. and Math. Phys., 5(1965), 1-80.

[37] A, Dubovickij, and A. Miljutin, Necessary Conditions for a Weak Extremum for the General Problem of Optimal Control, Nauka, Moscow, 1971, (Russian).

[38] D.B. Craven, Mathematical Programming and Control Theory, Champan and Hall, London, 1978.

[39] P. Gordan, Über die Auflösungen linearen Gleichungen mit reelen Coefficienten, Math. Annalen, 63(1873), 23-28.

[40] N. Girsanov, Lectures on the Mathematical Theory of Extremum Problems, Lecture Notes in Economics, 67(1972), Springer/Verlag, Berlin.

[41] E. Zeidler, Nonlinear Functional Analysis and its Applications-III, Springer-Verlag, New York-Berlin-Heidelberg-Tokyo, 1985, p.p. 662.

[42] S. Kurepa, Remarks on the Cauchy functional equations, Publ. Inst. Math. (Beograd), 19 (1965), 85-88.

[43] S. Kurepa, Quadratic functionals contained on an algebraic basic set, Glasnik Matematički, 26 (1971), 265-275.

[44] S. Kurepa: On Biomorphisms and Quadratic Forms on Groups, Aequations Mathematicae, 9 (1973), 30-45.

Milan R. TASKović

FACUlty of Mathematics

11000 Belgrade, P.O. Box 550

SERBIA

Home Address:

Milan R. TASKOvić

Nehruova 236

11070 BELGRADE

SERBIA

E-mail address: andreja@predrag.us 